\title{
Society for Immunotherapy of Cancer (SITC) clinical practice guideline on immunotherapy for the treatment of breast cancer
}

\author{
Leisha A Emens (D) , ${ }^{1}$ Sylvia Adams, ${ }^{2}$ Ashley Cimino-Mathews $(D),{ }^{3}$ Mary L Disis, ${ }^{4}$ \\ Margaret E Gatti-Mays (D) , ${ }^{5}$ Alice Y Ho, ${ }^{6}$ Kevin Kalinsky, ${ }^{7}$ Heather L McArthur, ${ }^{8}$ \\ Elizabeth A Mittendorf, ${ }^{9,10}$ Rita Nanda, ${ }^{11}$ David B Page (1) , ${ }^{12}$ Hope S Rugo (1) , ${ }^{13}$ \\ Krista M Rubin, ${ }^{14}$ Hatem Soliman, ${ }^{15}$ Patricia A Spears, ${ }^{16}$ Sara M Tolaney (1) , ${ }^{17}$ \\ Jennifer K Litton ${ }^{18}$
}

To cite: Emens LA, Adams $\mathrm{S}$, Cimino-Mathews A, et al. Society for Immunotherapy of Cancer (SITC) clinical practice guideline on immunotherapy for the treatment of breast cancer. Journal for ImmunoTherapy of Cancer 2021;9:e002597. doi:10.1136/jitc-2021-002597

Accepted 30 June 2021
Check for updates

(c) Author(s) (or their employer(s)) 2021. Re-use permitted under CC BY-NC. No commercial re-use. See rights and permissions. Published by BMJ.

For numbered affiliations see end of article.

Correspondence to Dr Jennifer K Litton; jlitton@mdanderson.org

Dr Leisha A Emens; emensla@upmc.edu

\section{ABSTRACT}

Breast cancer has historically been a disease for which immunotherapy was largely unavailable. Recently, the use of immune checkpoint inhibitors (ICls) in combination with chemotherapy for the treatment of advanced/metastatic triplenegative breast cancer (TNBC) has demonstrated efficacy, including longer progression-free survival and increased overall survival in subsets of patients. Based on clinical benefit in randomized trials, ICls in combination with chemotherapy for the treatment of some patients with advanced/metastatic TNBC have been approved by the United States (US) Food and Drug Administration (FDA), expanding options for patients. Ongoing questions remain, however, about the optimal chemotherapy backbone for immunotherapy, appropriate biomarker-based selection of patients for treatment, the optimal strategy for immunotherapy treatment in earlier stage disease, and potential use in histological subtypes other than TNBC. To provide guidance to the oncology community on these and other important concerns, the Society for Immunotherapy of Cancer (SITC) convened a multidisciplinary panel of experts to develop a clinical practice guideline (CPG). The expert panel drew upon the published literature as well as their clinical experience to develop recommendations for healthcare professionals on these important aspects of immunotherapeutic treatment for breast cancer, including diagnostic testing, treatment planning, immune-related adverse events (irAEs), and patient quality of life (QOL) considerations. The evidence-based and consensus-based recommendations in this CPG are intended to give guidance to cancer care providers treating patients with breast cancer.

\section{INTRODUCTION}

Both earlier detection and treatment based on identification of three major clinically relevant subtypes of breast cancer (ie, hormone receptor (HR) positive, human epidermal growth factor receptor 2 (HER2) positive and triple-negative) have improved outcomes for patients with breast cancer. ${ }^{1-5}$ Although breast cancer mortality has decreased by $40 \%$ since 1989, prognosis remains poor for patients who develop metastatic disease. For example, triple-negative breast cancer (TNBC) is associated with earlier age of onset and is more aggressive than other subtypes with a median survival of only 12-18 months in the metastatic setting. ${ }^{6}$ Historically, the therapeutic options for metastatic TNBC have been limited to standard chemotherapy, a strategy that typically results in the rapid emergence of chemotherapy-refractory disease. $^{78}$

In recent years, immunotherapy has emerged as a novel option for many difficult-to-treat cancers. In contrast to other solid tumors for which the role of immunotherapy is well-established, breast cancer has long been considered to be an immunologically 'cold' tumor, with relatively lower levels of $\mathrm{T}$ cell infiltration and lower mutational burdens compared to melanoma, non-small cell lung cancer, and other malignancies. ${ }^{9}$ More recently, the role of the immune system in both breast cancer progression and treatment response and resistance has come under critical re-evaluation, opening the door toward immunotherapeutic treatment. Retrospective analyses of tissue samples from clinical trials in breast cancer have revealed associations between lymphocytic infiltration into tumors and survival outcomes. ${ }^{7} 8$ 10-12 Furthermore, expression of the immune checkpoint proteins programmed cell death protein 1 and its ligand (PD-1 and PD-L1) within the tumor microenvironment ${ }^{1314}$ supports a role for breast cancer immunoediting. This is the three-phase process by which anti-cancer immune responses evolve to immune escape and disease progression. ${ }^{15} 16$ 
Clinical trials evaluating immune checkpoint inhibitor (ICI) therapies for TNBC have reported positive results. In 2019, the United States (US) Food and Drug Administration (FDA) granted accelerated approval of the PD-L1-directed antibody, atezolizumab, in combination with nanoparticle albumin-bound (nab) paclitaxel for advanced/metastatic PD-L1-positive (PD-L1+) TNBC, ${ }^{17}$ based on the results of the phase III IMpassion130 trial. ${ }^{18}$ Furthermore, in 2020, the FDA granted accelerated approval to the PD-1-directed antibody, pembrolizumab, in combination with chemotherapy for advanced/metastatic PD-L1+ TNBC, ${ }^{19}$ based on the results of the phase III KEYNOTE-355 trial. In 2021, the accelerated approval for pembrolizumab was converted to full approval and the accelerated approval for atezolizumab was voluntarily withdrawn. Ongoing clinical trials are investigating immunotherapies in other breast cancer subtypes as well as in early-stage disease, potentially expanding the population of patients with breast cancer who may benefit from immunotherapy.

Approval of anti-PD-(L) 1 agents for the treatment of breast cancer is relatively recent and, therefore, clinical experience with these new therapies is still somewhat limited. Immunotherapy, while offering survival benefits to some patients, is markedly different from conventional breast cancer therapies in several aspects including patient selection, treatment-related adverse events (AEs) including immune-related AEs (irAEs), and response patterns. To support the oncology community and provide evidence-based and consensus-based recommendations on immunotherapy for breast cancer, the Society for Immunotherapy of Cancer (SITC) convened a multidisciplinary panel of experts to develop a new clinical practice guideline (CPG), covering topics including recommended therapies, emerging agents, diagnostics and biomarkers, monitoring response to treatment, special patient populations, toxicity management, and quality of life (QOL). The recommendations within this guideline are not intended to supplant sound clinical judgment, but rather to provide clinicians with the most current thinking on how experts integrate immunotherapy into the treatment of patients with breast cancer.

\section{GUIDELINE DEVELOPMENT METHODS}

The Institute of Medicine's (IOM) Standards for Developing Trustworthy Clinical Practice Guidelines were used as a model to develop the recommendations in this manuscript. IOM standards dictate that guideline development is led by a multidisciplinary expert panel using a transparent process where both funding sources and conflicts of interest are readily reported. This CPG is intended to provide guidance and is not a substitute for the professional judgment of individual treating physicians.

\section{Conflict of interest management}

As outlined by IOM standards, all financial relationships of expert panel members that might result in actual, potential, or perceived conflicts of interest were individually reported. Disclosures were made prior to the onset of manuscript development and updated on an annual basis. In addition, panel members were asked to articulate any actual or potential conflicts at all key decision points during guideline development, so that participants would understand all possible influences, biases, and/or the diversity of perspectives on the panel. Although some degree of relationships with outside interests are to be expected among experts, panel candidates with significant financial connections that may compromise their ability to fairly weigh evidence (either actual or perceived) were not eligible to participate in guideline development.

Recognizing that guideline panel members are among the leading experts on the subject matter under consideration and guideline recommendations should have the benefit of their expertise, any identified potential conflicts of interests were managed as outlined in SITC's disclosure and conflict of interest resolution policies. As noted in these policies, panel members disclosing a real or perceived potential conflict of interest may be permitted to participate in consideration and decisionmaking of a matter related to that conflict, but only if deemed appropriate after discussion and agreement by the expert panel.

The financial support for the development of this guideline was provided solely by SITC. No commercial funding was received.

\section{Recommendation development}

Panel recommendations are based on literature evidence, where possible, and clinical experience, where appropriate. ${ }^{20}$ Consensus for the recommendations herein was generated by open communication and scientific debate in small-group and whole-group settings, surveying and responses to clinical questionnaires, as well as formal voting in consensus meetings.

For transparency, a draft of this CPG was made publicly available for comment during the development process and prior to publication. All comments were evaluated and considered for inclusion into the final manuscript according to the IOM standard.

\section{Evidence rating}

The evidence-based and consensus-based recommendations of the panel were refined throughout the development process in order to obtain the highest possible agreement among the experts, however, the minimum threshold was defined as $75 \%$ approval among the voting members. Evidence supporting panel recommendations was graded according to the Oxford Centre for EvidenceBased Medicine (OCEBM) Levels of Evidence Working Group 'The Oxford Levels of Evidence 2' (2016 version). A summary of the OCEBM grading scale may be found below (table 1). The level of evidence (LE) for a given recommendation is expressed in parentheses following 
Table 1 Summary of 'The Oxford Levels of Evidence 2'. (Adapted from Oxford Center for Evidence-Based Medicine Levels of Evidence Working Group)

\begin{tabular}{lllll}
\hline Level 1 & Level 2 & Level 3 & Level 4 & Level 5 \\
\hline $\begin{array}{l}\text { Systematic review or } \\
\text { meta-analysis }\end{array}$ & $\begin{array}{l}\text { Randomized trial or } \\
\text { observational study } \\
\text { with dramatic effect }\end{array}$ & $\begin{array}{l}\text { Non-randomized, } \\
\text { controlled cohort, or } \\
\text { follow-up study }\end{array}$ & $\begin{array}{l}\text { Case series, case- } \\
\text { control, or historically reasoning } \\
\text { controlled study }\end{array}$ & $\begin{array}{l}\text { Mechanism-based } \\
\text { red }\end{array}$ \\
\hline
\end{tabular}

the recommendation (eg, LE: 1). Recommendations without an associated LE were based on expert consensus.

\section{IMMUNOTHERAPY WITH PD-(L)1 INHIBITORS FOR THE TREATMENT OF ADVANCED/METASTATIC BREAST CANCER}

At the time of publication, two ICIs were FDA-approved specifically for the treatment of advanced/metastatic TNBC: atezolizumab and pembrolizumab. The indication for atezolizumab was withdrawn in 2021. Both breast cancer-specific approvals were for ICIs given in combination with cytotoxic chemotherapy, although the indicated backbone varies between agents and is an ongoing area of investigation. Pembrolizumab is also approved in a tissue-agnostic indication as monotherapy for tumors with high tumor mutational burden (TMB) or microsatellite instability (MSI). Landmark studies leading to FDA approvals for ICIs are summarized in table 2, along with select hypothesis-generating late-stage trials.

\section{FDA-approved indications: advanced TNBC}

The first ICI to be approved by the FDA for the treatment of breast cancer was atezolizumab, a fully humanized IgG1 isotype anti-PD-L1 monoclonal antibody $(\mathrm{mAb}) .^{21}$ Accelerated approval was granted in March 2019 for atezolizumab in combination with nab-paclitaxel for treatment of adult patients with PD-L1+ locally advanced or metastatic TNBC, as measured by the VENTANA PD-L1 (SP142) immunohistochemical (IHC) assay and assessed on immune cells (ICs) ${ }^{17}$; additional specifics of PD-L1 testing are described in detail in the Diagnostics and biomarker testing in patients with advanced/metastatic breast cancer section. The indication for atezolizumab for TNBC was voluntarily withdrawn in 2021. Although the approval did not specify line of therapy, data for the clinical activity of atezolizumab beyond the first-line setting is limited. However, in the phase Ib study of atezolizumab plus nab-paclitaxel for TNBC that led to the subsequent large scale trials, tolerable safety and promising activity was observed among 32 patients that received a median of three prior lines of therapy. ${ }^{22}$

The accelerated approval for atezolizumab was based on the first interim analysis of the phase III IMpassion 130 study, a multicenter, international, double-blind, placebocontrolled randomized trial. Enrollment criteria included patients with unresectable, locally advanced, or metastatic TNBC who had not received prior systemic therapy (radiation therapy and previous chemotherapy was allowed if treatment with curative intent was completed $\geq 12$ months before randomization). The study had four pre-specified co-primary endpoints: progression-free survival (PFS) in both the intent-to-treat (ITT) and PD-L1+ populations analyzed in parallel, and OS in both the ITT and PD-L1+ populations analyzed hierarchically, first in the ITT group, and then if significant in the PD-L1+ group. The trial randomized 902 patients in total, 451 in each arm. In the ITT population, 404 patients $(89.8 \%)$ in the atezolizumab group and 408 patients $(90.7 \%)$ in the placebo group had metastatic disease at baseline. ${ }^{18}$ In the primary analysis, IMpassion130 met its PFS endpoint in both the ITT and PD-L1+ populations (see table 2), although no benefit was observed in the PD-L1-negative group. ${ }^{23}$ For OS, a statistically significant benefit was not seen in the ITT subgroup, so formal statistical testing could not be performed in the PD-L1+ subgroup per the hierarchical statistical analysis plan. However, exploratory analyses demonstrated a clinically meaningful improvement in OS with ICI compared with placebo in the PD-L1+ subgroup of 9.5 and 7 months at the first and second interim OS analyses, respectively. ${ }^{24}$ In the final OS analysis, there was a 7.5-month improvement in OS for the PD-L1+ subgroup, reflecting a HR of 0.67 (95\% confidence interval [CI] 0.53 to 0.86$).{ }^{25}$ Notably, the data for the PD-L1+ subgroup remained consistent in the first, second and final OS analyses with a final HR of 0.67 and a final OS improvement of 7.5 months at a median follow-up of 18.8 months-a clinically meaningful benefit. A follow-up phase III randomized study, IMpassion131, which investigated the addition of atezolizumab to paclitaxel (described in more detail in the Emerging data on PD-(L) 1 inhibitors for recurrent/metastatic breast cancer section) did not demonstrate benefit, for reasons that remain unclear and require further investigation.

KEYNOTE-355 was a randomized, double-blind, phase III study of the anti-PD-1 mAb pembrolizumab combined with chemotherapy (physician's choice of paclitaxel, nab-paclitaxel, or carboplatin plus gemcitabine) versus placebo and chemotherapy for previously untreated, locally recurrent, inoperable, or metastatic TNBC. Eligibility included patients who had recurrent disease $\geq 6$ months from completion of adjuvant therapy. In the trial, PD-L1 status was determined by the PD-L1 IHC 22C3 pharmDx assay, which assesses expression on both tumor cells (TCs) and ICs, resulting in a combined positive score (CPS), which is the number of PD-L1 staining cells (TCs, lymphocytes, macrophages) divided by the total number of viable TCs, multiplied by 100 (see the Diagnostics and biomarker testing for patients with advanced/ metastatic breast cancer section for additional details on 
Table 2 Trials of ICls for recurrent/metastatic breast cancer and tissue-agnostic indications

\begin{tabular}{|c|c|c|c|c|}
\hline Trial name & Phase & Setting & $\begin{array}{l}\text { Control and immunotherapy } \\
\text { arms }\end{array}$ & $\begin{array}{l}\text { Key outcome measures for } \\
\text { FDA approval }\end{array}$ \\
\hline \multicolumn{5}{|c|}{ Trials leading to FDA approvals } \\
\hline KEYNOTE-355 & III & $\begin{array}{l}\text { Previously } \\
\text { untreated TNBC }\end{array}$ & $\begin{array}{l}\text { Control }(n=281) \text { : } \\
\text { Placebo+investigator's choice: } \\
\text { nab-paclitaxel, paclitaxel, or } \\
\text { gemcitabine+ carboplatin } \\
\text { Immunotherapy ( } n=566) \text { : } \\
\text { Pembrolizumab+investigator's } \\
\text { choice: nab-paclitaxel, paclitaxel, } \\
\text { or gemcitabine+ carboplatin }\end{array}$ & $\begin{array}{l}\text { CPS } \geq 10 \\
\text { PFS } 9.7 \text { vs } 5.6 \text { months } \\
\text { HR } 0.65 \\
(95 \% \mathrm{Cl} 0.49 \text { to } 0.86 ; \\
p=0.0012) \\
\text { CPS } \geq 1 \\
\text { PFS } 7.6 \text { vs } 5.6 \text { months } \\
\text { HR } 0.74 \\
(95 \% \mathrm{Cl} 0.61 \text { to } 0.90 ; \\
p=0.0014)\end{array}$ \\
\hline \multicolumn{5}{|c|}{ Hypothesis-generating trials } \\
\hline KEYNOTE-119 & III & $\begin{array}{l}\text { TNBC that has } \\
\text { progressed on } \\
\text { prior therapy }\end{array}$ & $\begin{array}{l}\text { Control }(n=310) \text { : Investigator's } \\
\text { choice: capecitabine, eribulin, } \\
\text { gemcitabine, or vinorelbine } \\
\text { Immunotherapy }(n=312) \text { : } \\
\text { Pembrolizumab }\end{array}$ & $\begin{array}{l}\text { CPS } \geq 10 \\
\text { OS } 12.7 \text { vs } 11.6 \text { months } \\
\text { HR } 0.78 \\
\text { ( } 95 \% \mathrm{Cl} 0.57 \text { to } 1.06 ; \\
p=0.0574) \\
\text { CPS } \geq 1 \\
\text { OS } 10.7 \text { vs } 10.2 \text { months } \\
\text { HR } 0.86 \\
\text { ( } 95 \% \mathrm{Cl} 0.69 \text { to } 1.06 ; \\
p=0.0728) \\
\text { ITT } \\
\text { OS } 9.9 \text { vs } 10.8 \text { months } \\
\text { HR } 0.97 \\
\text { ( } 95 \% \mathrm{Cl} 0.82 \text { to } 1.15 \text { ) }\end{array}$ \\
\hline IMpassion131 & III & $\begin{array}{l}\text { Previously } \\
\text { untreated TNBC }\end{array}$ & $\begin{array}{l}\text { Control }(n=220) \text { : Placebo+paclitaxel } \\
\text { Immunotherapy }(n=431) \text { : } \\
\text { Atezolizumab+paclitaxel }\end{array}$ & $\begin{array}{l}\text { PD-L1 IC+ } \\
\text { PFS } 6 \text { vs } 5.7 \text { months } \\
\text { HR } 0.82 \\
(p=0.20) \\
\text { ITT } \\
\text { OS } 19.2 \text { vs } 22.8 \text { months } \\
\text { HR } 1.11\end{array}$ \\
\hline KATE2 & II & $\begin{array}{l}\text { HER2+breast } \\
\text { cancer with prior } \\
\text { trastuzumab and } \\
\text { taxane therapy }\end{array}$ & $\begin{array}{l}\text { Control }(n=69) \text { : } \\
\text { Placebo+trastuzumab emtansine } \\
\text { Immunotherapy }(n=133) \text { : } \\
\text { Atezolizumab+trastuzumab } \\
\text { emtansine }\end{array}$ & $\begin{array}{l}\text { ITT } \\
\text { Median PFS } \\
8.2 \text { vs } 6.8 \text { months } \\
\text { HR } 0.82 \\
(95 \% \mathrm{Cl} 0.55 \text { to } 1.23 ; p=0.33 \text { ) }\end{array}$ \\
\hline
\end{tabular}

\section{Trials leading to tissue-agnostic approvals}

Pooled analysis: $\quad$ Multi-cohort, MSI-H or dMMR Immunotherapy ( $n=149$; five KEYNOTE-016 KEYNOTE-164 KEYNOTE-012 KEYNOTE-028 KEYNOTE-158 single-arm tumors that have patients with breast cancer): progressed on Pembrolizumab prior therapy 
Table 2 Continued

\begin{tabular}{|c|c|c|c|c|}
\hline Trial name & Phase & Setting & $\begin{array}{l}\text { Control and immunotherapy } \\
\text { arms }\end{array}$ & $\begin{array}{l}\text { Key outcome measures for } \\
\text { FDA approval }\end{array}$ \\
\hline KEYNOTE-158 & $\begin{array}{l}\text { Multi-cohort, } \\
\text { single-arm }\end{array}$ & $\begin{array}{l}\text { TMB-H tumors } \\
\text { ( } \geq 10 \text { mut/ } \\
\text { Mb) that have } \\
\text { progressed on } \\
\text { prior therapy }\end{array}$ & $\begin{array}{l}\text { Immunotherapy ( } \mathrm{n}=102 ; 0 \\
\text { patients with breast cancer): } \\
\text { Pembrolizumab }\end{array}$ & $\begin{array}{l}\text { ORR } 29 \% \\
\text { (95\% Cl } 21 \% \text { to } 39 \%) \\
\text { CR rate } 4 \% \\
\text { Median DOR not reached } \\
\text { (57\% lasting } \geq 12 \text { months; } 50 \% \\
\text { lasting } \geq 24 \text { months) }\end{array}$ \\
\hline
\end{tabular}

$\mathrm{Cl}$, confidence interval; CPS, combined positive score; CR, complete response; dMMR, mismatch-repair deficient; DOR, duration of response; FDA, Food and Drug Administration; HR, hazard ratio; IC, immune cell; ITT, intent-to-treat; MSI-H, microsatellite instability high; ORR, overall response rate; OS, overall survival; PD-L1, programmed death ligand 1; PFS, progression-free survival; R/M, recurrent/ metastatic; TMB-H, high tumor mutation burden; TNBC, triple-negative breast cancer.

PD-L1 testing). At a median follow-up of 17.5 months for the pembrolizumab arm $(\mathrm{n}=566)$ and 15.5 months for the chemotherapy arm $(n=281)$, significant PFS benefit was observed for patients with CPS $\geq 10 \quad(n=636)$ tumors. In the CPS $\geq 1$ group ( $n=323$ ), PFS also numerically increased with pembrolizumab (see table 2), although this did not reach the pre-specified threshold for statistical significance. $^{26}$ Benefit was observed regardless of whether patients received a taxane or gemcitabine and carboplatin. Although this analysis was exploratory only and the study was not powered to compare the regimens, the HRs in the CPS $\geq 10$ population for nab-paclitaxel $(n=99)$, paclitaxel $(\mathrm{n}=44)$, and gemcitabine and carboplatin $(\mathrm{n}=180)$ were 0.57 (95\% CI 0.34 to 0.95$), 0.33$ (95\% CI 0.14 to 0.76 ), and 0.77 (95\% CI 0.53 to 1.11 ). ${ }^{27}$ Formal testing for PFS was not performed in the ITT population. According to a press release in July 2021, KEYNOTE-355 met its primary survival endpoint with pembrolizumab demonstrating a statistically significant and clinically meaningful improvement in OS for patients whose tumors expressed PD-L1 with a combined positive score $\geq 10$ compared to chemotherapy alone. Based on these data, the FDA granted accelerated approval to pembrolizumab in combination with chemotherapy in November 2020 for the treatment of patients with locally recurrent, unresectable, or metastatic TNBC whose tumors express PD-L1 with CPS $\geq 10$ as determined by an FDA-approved test. Accelerated approval was converted to full (regular) approval by the FDA in July 2021. Similar to atezolizumab, the approval does not specify line of therapy nor chemotherapy backbone.

\section{Tissue-agnostic FDA approvals for checkpoint inhibitors}

Pembrolizumab is approved for two 'tissue agnostic' (ie, irrespective of primary site of origin) indications, based on high level evidence that tumor neoantigens elicit cytotoxic T cell responses. ${ }^{28-30}$ Somatic mutations give rise to mutant proteins that are proteolytically processed and presented on major histocompatibility complex Class I (MHC Class I) molecules. Therefore, TMB is generally considered a surrogate for neoantigen load and a predictive biomarker for T cell reactivity. ${ }^{3132}$ One common driver for a highly mutagenic tumor phenotype is a deficiency in one or more components of the mismatch repair (MMR) machinery. MMR deficient (dMMR) tumors frequently display a molecular signature characterized by spontaneous loss or gain of nucleotides in repetitive sequences, and instability in five or more loci is defined as MSI-high (MSI-H) ${ }^{33}$ Full FDA approval of pembrolizumab for the treatment of MSI-H or dMMR tumors that have progressed on prior therapy regardless of tissue of origin, was first issued in May 2017..$^{34}$ This approval was based on durable responses among 149 patients with 15 different tumor types in five single-arm multicohort multicenter trials: KEYNOTE-016, ${ }^{35}$ KEYNOTE-164, ${ }^{36}$ KEYNOTE-012, ${ }^{37}$ KEYNOTE-028, ${ }^{38}$ and KEYNOTE-158 (which included five patients with histologically/cytologically confirmed MSI-H/dMMR advanced breast cancer) ${ }^{39}$ (see table 2). Pembrolizumab was also approved for non-MSI-H/dMMR tumors with high mutation burden (TMB-H) based on KEYNOTE-158 in June 2020. TMB-H was defined in this study as $\geq 10$ mutations per megabase (mut/Mb) as assayed by the FoundationOne CDx companion diagnostic-no patients with breast cancer were included in the analysis that led to approval, however. ${ }^{40}$

It is important to note that breast cancers are rarely MSI-H. Current data suggest that roughly $1 \%$ of TNBC ${ }^{41}$ and fewer than $2 \%$ of breast cancers overall are MSI-H. ${ }^{42}$ In addition, although mutation burdens vary across subtypes, with relatively higher mutation frequencies observed in HER2+ tumors and TNBC, ${ }^{43-45}$ TMB-H is also infrequent in breast cancer. One analysis of 3,969 tumor samples across breast cancer subtypes estimated an overall rate of roughly $5 \%$ TMB-H tumors, with slightly higher incidence in metastatic sites compared with the primary lesions. ${ }^{46}$ TMB-H has been associated with improved outcomes in patients with breast cancer receiving immunotherapy, however, benefit may be contingent on additional tumor properties, such as PD-L1 status. ${ }^{47}$ In the phase II TAPUR trial, a basket study evaluating commercially available targeted agents in patients with advanced cancers with specific genomic alterations, 28 women with metastatic breast cancer and tumors with mutation burdens ranging from 9 to 37 muts/Mb received pembrolizumab. All patients had received at least two prior lines of systemic 
Table 3 Association with TMB and benefit with ICls in KEYNOTE-119 and IMpassion130

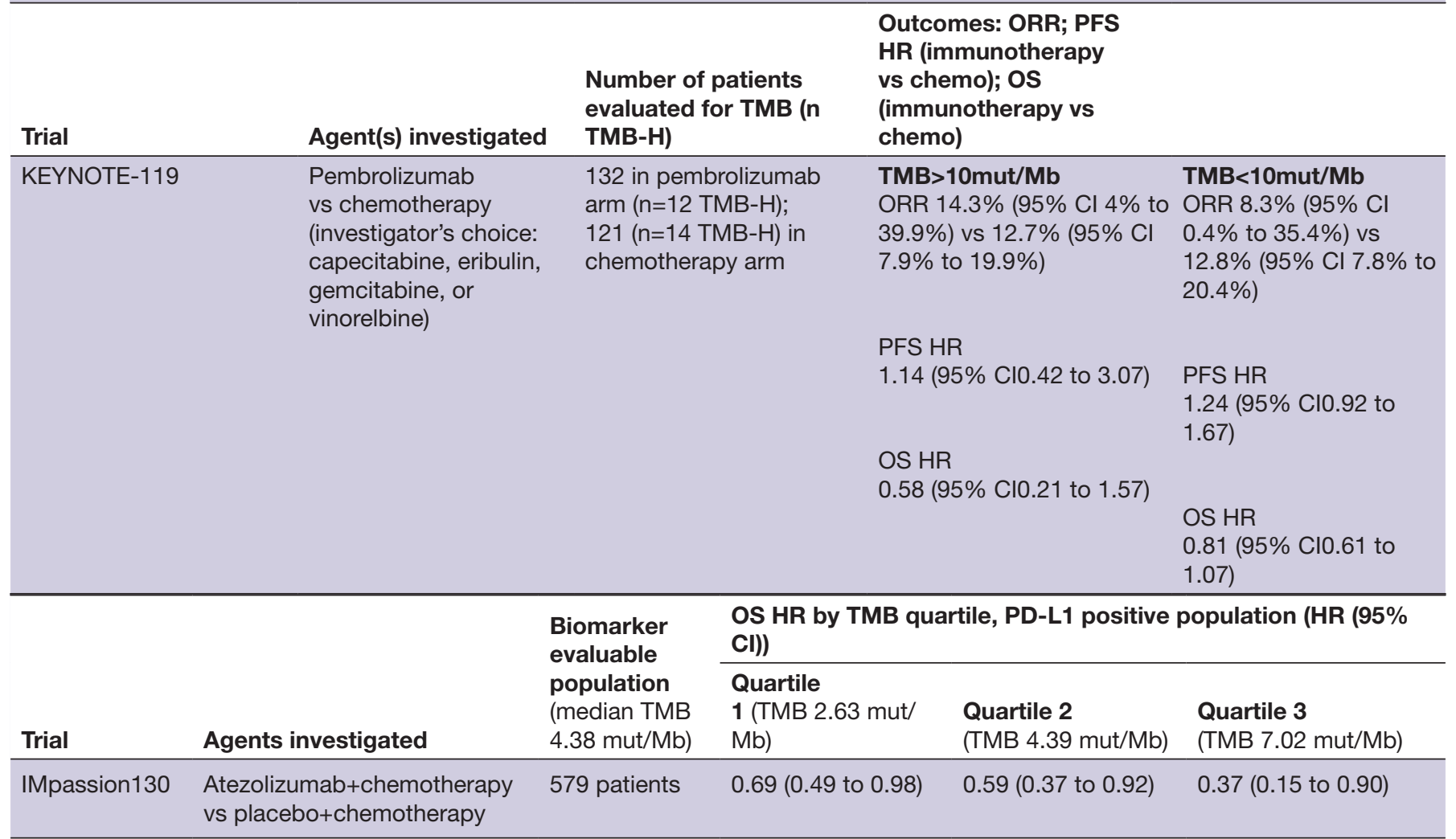

chemo, chemotherapy; $\mathrm{Cl}$, confidence interval; HR, hazard ratio; mut/Mb, mutations per megabase; ORR, overall response rate; OS, overall survival; PD-L1, programmed death-ligand 1; PFS, progression-free survival; TMB, tumor mutational burden; TMB-H, high TMB.

treatment, with 26 (93\%) having been previously treated with three or more therapies. The overall response rate (ORR) was $21 \%$ (95\% CI $8 \%$ to $41 \%$ ), with a median PFS of 10.6 weeks (95\% CI 7.7 to 21.1) and a median OS of 31.6 weeks (95\% CI 11.9 to not estimable). No association was observed between increasing TMB and PFS or OS. ${ }^{48}$

Data sets from phase II and III TNBC trials are currently being analyzed retrospectively to determine the prognostic value of TMB for ICI therapy. In KEYNOTE-119, ${ }^{49}$ high TMB was associated with increased clinical benefit with immunotherapy. Similarly, in IMpassion130, increasing TMB was also associated with improved PFS (highest TMB quartile HR 0.56 [95\% CI 0.38 to 0.81 ]), but the association was primarily driven by the PD-L1+ subgroup (HR 0.31 [95\% CI 0.17 to 0.57 ] vs 0.84 [95\% CI 0.48 to 1.47 ] for PD-L1-negative cases). ${ }^{47}$ Key outcomes for KEYNOTE-119 and IMpassion130 are summarized in table 3 .

\section{Emerging data on PD-(L)1 inhibitors for advanced/metastatic breast cancer}

Several additional ICI-based approaches are currently under investigation for the treatment of advanced/metastatic breast cancer, including monotherapy regimens, combinations with chemotherapy, and combinations with biologics. Results of emerging approaches that have advanced through to later-phase trials are discussed below and summarized in table 4 . For further information on additional strategies limited to early-phase trials, such as immunotherapy regimens that include targeted agents such as poly ADP-ribose polymerase (PARP), radiation therapy, CDK4/ 6 inhibitors, and AKT inhibitors, see the Novel combination strategies and promising future directions section.

\section{Advanced/metastatic TNBC}

The optimal chemotherapy backbone for immunotherapycontaining regimens is unknown, however current data suggest that paclitaxel is not indicated in combination with atezolizumab for advanced/metastatic TNBC. IMpassion131, a phase III placebo-controlled study, compared the efficacy and safety of first-line atezolizumab combined with paclitaxel versus placebo with paclitaxel. ${ }^{50}$ In the trial, adding atezolizumab to paclitaxel did not improve PFS or OS in either the PD-L1+ or the ITT population. ${ }^{51}$ On September 8, 2020, the FDA issued an alert to oncology professionals stating that the combination of atezolizumab and paclitaxel did not significantly reduce the risk of cancer progression and death compared with placebo and paclitaxel in the PD-L1+ population, and healthcare providers were directed not to replace nabpaclitaxel with paclitaxel in clinical practice. ${ }^{52}$ Additional trials evaluating different chemotherapy backbones are ongoing, including the randomized, placebo-controlled, phase III IMpassion132 study, which is evaluating atezolizumab with capecitabine or gemcitabine/carboplatin for 
inoperable locally advanced/metastatic TNBC recurring $\leq 12$ months after completing standard neoadjuvant and/ or adjuvant anthracycline-based and taxane-based chemotherapy or definitive surgery, whichever occurred last. ${ }^{53}$

Pembrolizumab has been evaluated as monotherapy in multiple trials for TNBC. The KEYNOTE-086 phase II study enrolled two cohorts of patients, one who had undergone prior treatment with anthracycline and taxane in any disease setting with progression on or after the most recent therapy, and another with no prior systemic therapy for metastatic disease. Patients in the cohort treated with first-line pembrolizumab were required to have PD-L1+ tumors defined as CPS $\geq 1$ by the 22C3 assay, ${ }^{54}{ }^{55}$ for whom an ORR of $21.4 \%$ was subsequently demonstrated (95\% CI $13.9 \%$ to $31.4 \%$ ). ${ }^{56}$ For the 170 patients with previously treated advanced TNBC, ORR was $5.3 \%$ (95\% CI $2.7 \%$ to $9.9 \%$ ) in the total population and $5.7 \%$ (95\% CI 2.4\% to $12.2 \%$ ) in the PD-L1+ populations. Median PFS was 2 months (95\% CI 1.9 to 2) and median OS was 9 months (95\% CI 7.6 to 11.2) for all patients. ${ }^{57}$ In KEYNOTE-119, patients with metastatic TNBC who had received one to two prior systemic therapies were randomized to receive pembrolizumab $(n=312)$ or physician's choice of capecitabine, eribulin mesylate, gemcitabine, or vinorelbine $(n=310)$. Patients were stratified by PD-L1 CPS. At a median follow-up of 9.9 months for the pembrolizumab group and 10.9 months for the chemotherapy group, single-agent pembrolizumab did not significantly improve OS compared with singleagent chemotherapy in the ITT population nor the prespecified subgroups. In an exploratory analysis of patients with CPS $\geq 20$, median OS was 14.9 months with pembrolizumab versus 12.5 months with chemotherapy (HR 0.58 ; $95 \%$ CI 0.38 to 0.88$).{ }^{58}$

\section{Advanced HER2+ breast cancer}

Signals of clinical efficacy have been reported with the addition of ICIs to standard of care therapies in HER2+ advanced breast cancer. Beyond immunotherapy, additional targeted agents such as trastuzumab deruxtecan and tucatinib continue to offer more options to patients with HER2+ disease. ${ }^{5960} \mathrm{In}$ the phase II KATE2 study, which randomized 133 patients to receive atezolizumab plus trastuzumab emtansine (T-DM1) and 69 patients to receive placebo plus T-DM1, no statistically significant difference in overall PFS was observed between the two arms. A trend toward more favorable PFS and ORR were seen with the combination in patients with tumor infiltrating lymphocyte (TIL) $\geq 5 \%$ and/or PD-L1+ tumors as defined by an IC score $\geq 1$ by the SP142 assay. ${ }^{61}$ Updated data with a median follow-up of 19.5 months for the atezolizumab arm and 18.2 months for the placebo arm revealed similar 1-year OS rates in both arms $(89.1 \%$ vs $89 \%$ for atezolizumab vs placebo; HR $0.74 ; 95 \%$ CI 0.42 to 1.30 ). In the PD-L1+ subgroup $(n=57$ in the atezolizumab arm and $n=27$ in the placebo arm), the 1-year OS was numerically greater in the atezolizumab arm compared with placebo $(94.3 \%$ vs $87.9 \%$; HR $0.55 ; 95 \%$ CI 0.22 to 1.38$).{ }^{61}$ A definitive phase III trial is planned based on this hypothesis-generating data.
Providing further support for additional investigation of ICIs in HER2+ disease, the phase Ib/II PANACEA study explored pembrolizumab in combination with trastuzumab in patients with HER2+, trastuzumab-resistant metastatic breast cancer. Of the 52 heavily pre-treated patients enrolled in the phase II portion, 46 patients $(77 \%)$ had PD-L1+ disease (CPS $\geq 1 \%$ ), and, of these, $7(15 \%)$ achieved an objective response and $4(8 \%)$ maintained stable disease (SD) for more than 6 months. ${ }^{62}$

\section{Advanced ER+ breast cancer}

KEYNOTE-028 was a phase Ib, open-label, multicohort study that investigated the safety and antitumor activity of pembrolizumab in patients with PD-L1+ advanced solid tumors, including 25 patients with estrogen receptor positive (ER+) /HER2-negative (HER2-) advanced breast cancer, among whom three experienced partial response (PR), leading to an ORR of $12 \%$ (95\% CI $2.5 \%$ to $31.2 \%$ ). The clinical benefit rate (complete response $(\mathrm{CR})+\mathrm{PR}+\mathrm{SD}$ (for $\geq 24$ weeks)) was $20 \%$ (95\% CI $7 \%$ to $41 \%$ ) and the median duration of response (DOR) was 12 months (range 7.4 to 15.9 months).$^{38}$ In the phase Ib JAVELIN study, which evaluated the anti-PD-L1 avelumab in 72 women with HR+/ HER2- disease (with no requirements for PD-L1 expression), an ORR of $2.8 \%$ was observed. ${ }^{63}$

Pembrolizumab has been evaluated in combination with eribulin mesylate for ER+ metastatic breast cancer in a randomized phase II trial that enrolled 88 patients. At a median follow-up of 10.5 months, no significant difference in median PFS and ORR was observed with the addition of pembrolizumab to eribulin mesylate compared with eribulin mesylate alone (PFS, 4.1 vs 4.2 months; HR 0.80; HR 95\% CI 0.50 to $1.26 ; \mathrm{p}=0.33$; ORR $27 \%$ vs $34 \%$, respectively; $\mathrm{p}=0.49$ ). PD-L1 testing by the 22C3 assay was performed for 65 patients, and $24(36.9 \%)$ were found to have PD-L1+ tumors (modified proportion score $>1 \%$ ). PD-L1 status was not associated with PFS, although the group of patients with PD-L1+ tumors was small and thus had limited power to assess benefit. ${ }^{64}$

\section{Panel recommendations}

- Clinical trial enrollment remains a priority to further understand the benefit of checkpoint inhibition in metastatic breast cancer.

- All patients with unresectable locally advanced or metastatic TNBC should have tumor tissue tested for PD-L1 by an FDA-approved assay for breast cancer.

- All patients with locally advanced or metastatic breast cancer should undergo comprehensive genomic profiling, including testing for TMB and MSI.

- With the withdrawal of the indication for atezolizumab with nab-paclitaxel in metastatic TNBC, one companion diagnostic is approved by the FDA for PD-L1 testing in metastatic TNBC: the 22C3 assay with tumor and IC scoring by combined positive score. Benefit is seen for adding pembrolizumab to chemotherapy in patients with tumors expressing PD-L1 by CPS score $\geq 10$ (LE:2). 
- For patients with locally advanced/metastatic TNBC and PD-L1+ tumors by CPS score $\geq 10$ using the 22C3 assay, pembrolizumab plus nab-paclitaxel, paclitaxel, or carboplatin and gemcitabine is recommended as one immunotherapy option for first-line treatment (LE:2), based on clinically meaningful PFS improvement in KEYNOTE-355.

- For patients with locally advanced/metastatic TNBC, pembrolizumab should only be added to chemotherapy (nab-paclitaxel, paclitaxel or carboplatin/ gemcitabine combination) if tumors express PD-L1 with CPS $\geq 10$ by the 22C3 assay (until PD-L1 assays are harmonized) (LE: 2).

- For patients with locally advanced/metastatic TNBC and PD-L1+ tumors being treated with atezolizumab, nab-paclitaxel is the only chemotherapy backbone that has demonstrated activity in randomized clinical trials (LE: 2). The indication for atezolizumab in this setting was voluntarily withdrawn in 2021.

- All patients who are candidates for immunotherapy treatment for metastatic TNBC should have tumor tissue tested for PD-L1 at least once, irrespective of line of therapy or prior immunotherapy in the adjuvant or neoadjuvant setting.

- Patients deriving clinical benefit from atezolizumabbased treatment in the absence of clinically significant toxicity or disease progression should continue on atezolizumab plus nab-paclitaxel rather than change therapy.

\section{EMERGING DATA ON IMMUNOTHERAPY WITH PD-(L)1 INHIBITORS FOR EARLY-STAGE/LOCALLY ADVANCED BREAST CANCER}

At the time of guideline writing, one ICI, pembrolizumab, was approved by the FDA for the treatment of patients with high-risk early-stage TNBC in combination with chemotherapy as neoadjuvant treatment and then continued as a single agent as adjuvant treatment after surgery. The potential for permanent irAEs must be considered in the risk-benefit calculation when discussing immunotherapy with a patient with early stage disease who is treated with curative intent. Immunotherapy for early-stage breast cancer is an active and rapidly evolving area of research. Several trials have been completed evaluating various ICI and chemotherapy regimens in the neoadjuvant setting. Results of completed trials are summarized in table 4 .

\section{Neoadjuvant setting}

The phase II I-SPY 2 trial indicated that the addition of pembrolizumab to standard neoadjuvant chemotherapy improved estimated pCR rates in patients with high-risk stage II/III TNBC and Mammaprint-defined high-risk HR+/HER2- breast cancer. I-SPY 2 is a platform trial with an adaptive design that evaluates multiple investigational agents concurrently against a common control arm of weekly paclitaxel for 12 weeks followed by doxorubicin plus cyclophosphamide given every 2-3 weeks for 4 cycles. 'Graduation' for efficacy occurs if the predefined efficacy threshold of $85 \%$ probability of success in a subtype-specific, hypothetical 300 patient phase III trial is met. The primary endpoint of the I-SPY 2 trial is pCR rate. The first immunotherapy arm investigated the efficacy of 4 cycles of pembrolizumab added to neoadjuvant paclitaxel followed by adjuvant chemotherapy. ${ }^{65}$ In a recent report including 250 patients from I-SPY 2 randomized to standard chemotherapy with or without pembrolizumab, the addition of pembrolizumab to paclitaxel demonstrated improved estimated pCR rates across TNBC and ER+ subtypes compared with standard chemotherapy. For the 69 patients receiving pembrolizumab (40 $\mathrm{HR}+$ / HER2- and 29 TNBC), the final estimated pCR rates in the TNBC cohort were $60 \%$ versus $22 \%$ for pembrolizumab versus control. The estimated pCR rates were $30 \%$ versus $13 \%$ in the HR+/HER2- cohort and $44 \%$ versus $17 \%$ among all HER2- patients. ${ }^{65}$

In the I-SPY2 trial, pembrolizumab 'graduated' for both HR+/HER2- and TNBC after 69 patients had been randomized to receive the investigational therapy and 201 were randomized to the control group. Pembrolizumab was the first of 10 agents to graduate for HR+/HER2disease. A second arm investigated 8 cycles of pembrolizumab, half of which were given without chemotherapy. ${ }^{66}$ In this arm, patients received paclitaxel plus pembrolizumab for 4 cycles followed by 4 cycles of pembrolizumab alone, without adjuvant chemotherapy. Of the 73 patients who were randomized to this arm, three progressed while receiving pembrolizumab alone. Treatment with pembrolizumab alone was no longer allowed due to the potential concern for progression for those randomized to pembrolizumab alone, and investigators were given the option to administer adjuvant chemotherapy with pembrolizumab or proceed with definitive surgery following the 12 weeks of paclitaxel plus pembrolizumab. The estimated pCR rates for the HR+/HER2- and TNBC signatures were the same for the pembrolizumab versus control arms, at $15 \%$ (95\% CI $10 \%$ to $29 \%$ ) versus $15 \%$ (95\% CI $9 \%$ to $20 \%)$ and $27 \%$ (95\% CI $9 \%$ to $45 \%$ ) versus $27 \%$ (95\% CI $19 \%$ to $50 \%$ ), respectively. ${ }^{66}$

Six different pembrolizumab plus chemotherapy regimens were evaluated as neoadjuvant therapy for high-risk, early-stage TNBC in the phase Ib trial KEYNOTE-173. All cohorts received a single run-in dose of pembrolizumab for cycle one, followed by 8 cycles of pembrolizumab in combination with a taxane (nab-paclitaxel for five of six cohorts and paclitaxel for the remaining cohort) with or without carboplatin at varying dosing levels for 12 weeks. For an additional 12 weeks before surgery, all patients received doxorubicin and cyclophosphamide. The overall pCR rate for all cohorts was $60 \%$ (90\% CI $30 \%$ to $85 \%$ ), with ORR ranging from $70 \%$ to $100 \%$ in the different chemotherapy dosing regimens. At a median follow-up of 19.6 months, the 12-month EFS rate was $100 \%$ and $88 \%$ for patients who did and did not achieve pCR, respectively. Four cohorts (three groups who received pembrolizumab with nab-paclitaxel and carboplatin as well as the group receiving paclitaxel plus carboplatin) 
Table 4 Completed phase II/III neoadjuvant immunotherapy trials for early-stage breast cancer

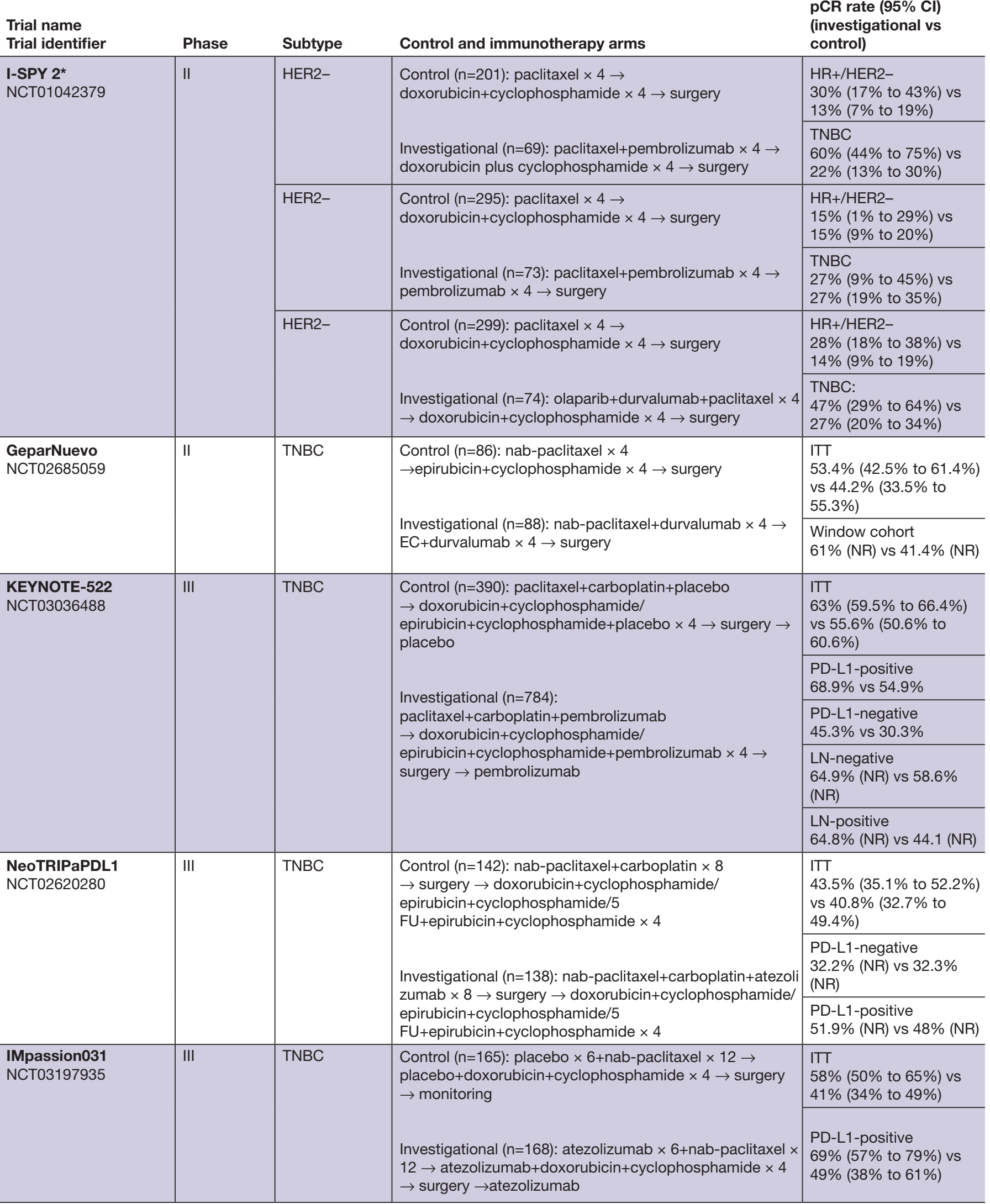

${ }^{*} \mathrm{pCR}$ rate in I-SPY 2 trial is estimated due to adaptive clinical trial design.

EC, epirubicin/cyclophosphamide; HER2, human epidermal growth factor receptor 2; HR, hormone receptor; ITT, intent-to-treat; LN, lymph node; NR, not reported; pCR, pathologic complete response; PD-L1, programmed death-ligand 1; TNBC, triple-negative breast cancer. 
had a 12-month EFS rate of $100 \% .{ }^{67}$ In exploratory analyses, significant associations with $\mathrm{pCR}$ rates were observed for pre-treatment PD-L1 CPS ( $\mathrm{p}=0.0127)$, and both pretreatment and on-treatment stromal TILs $(\mathrm{p}=0.0059$ and 0.0085 , respectively). ${ }^{68}$ The results of KEYNOTE- 173 informed the chemotherapy backbone selection for the subsequent phase III KEYNOTE-522 trial.

In July 2021, the FDA granted regular approval to pembrolizumab for the treatment of patients with highrisk TNBC in combination with chemotherapy as neoadjuvant treatment and then continued as a single agent as adjuvant treatment after surgery. Approval was based on KEYNOTE-522, a randomized phase III trial, which assigned patients with previously untreated stage II or stage III TNBC in a 2:1 ratio to receive neoadjuvant therapy with 4 cycles of pembrolizumab $(200 \mathrm{mg}$ ) every 3 weeks plus paclitaxel and carboplatin ( $n=784$ patients) or placebo every 3 weeks plus paclitaxel and carboplatin ( $\mathrm{n}=390$ patients). Both groups received an additional 4 cycles of pembrolizumab or placebo, and both groups received doxorubicin+cyclophosphamide or epirubicin+cyclophosphamide before surgery. After definitive surgery, the patients received adjuvant pembrolizumab or placebo every 3 weeks for up to 9 cycles. At the first interim analysis of the initial 602 randomized patients, the pCR rate was $64.8 \%$ (95\% CI $59.9 \%$ to $69.5 \%$ ) in the group receiving pembrolizumab and $51.2 \%$ (95\% CI $44.1 \%$ to $58.3 \%$ ) in the group receiving placebo $(\mathrm{p}<0.001)$. In the trial, improved pCR rates with pembrolizumab were generally consistent across subgroups, regardless of tumor size or PD-L1 status. The increase in pCR rate with the addition of pembrolizumab was numerically greater for patients with node positive disease (20.6\% increase; $95 \%$ CI $8.9 \%$ to $31.9 \%$ ) than for those without lymph node involvement (6.3\% increase; $95 \%$ CI $-5.3 \%$ to $18.2 \%) .{ }^{69}{ }^{70}$ Recently released data from the third pre-planned interim analysis including the entire ITT population $(\mathrm{n}=1174)$ showed pCR rates of $63 \%$ (95\% CI 59.5 to 66.4 ) and $55.6 \%(95 \%$ CI 50.6 to 60.6 ) in the pembrolizumab and placebo arms, respectively, for a stratified delta of $7.5 \%$ (95\% CI 1.6 to 13.4). At a median follow-up of 26.1 months with roughly $53 \%$ of required events, the EFS HR was 0.65 (95\% CI 0.48 to 0.88$).^{71}$ The fourth pre-planned interim analysis was presented during an online ESMO Virtual Plenary session on July $15,2021 .^{72}$ With a median follow-up of 39.1 months, the addition of pembrolizumab to chemotherapy resulted in a statistically significant and clinically meaningful improvement in EFS, with a HR of 0.63 (95\% CI 0.48 to $0.82 ; \mathrm{p}=0.00031$ ). No new safety signals emerged. Most immune-mediated AEs were low-grade, occurred in the neoadjuvant phase, and were manageable with treatment interruption, steroid administration, and/or hormone replacement. Treatment-related AEs of grade $\geq 3$ occurred in $77.1 \%$ of the patients who received pembrolizumab compared with $73.3 \%$ of the patients in the placebo group, leading to discontinuation of any trial drug in $27.7 \%$ and $14.1 \%$ in each arm, respectively. The most common irAEs reported among patients receiving pembrolizumab (incidence $\geq 10$ patients) were infusion reactions (18\%), hypothyroidism $(15.1 \%)$, severe skin reactions $(5.7 \%)$, hyperthyroidism $(5.2 \%)$, adrenal insufficiency $(2.4 \%)$, pneumonitis $(2.2 \%)$, thyroiditis $(2.0 \%)$, hypophysitis $(1.9 \%)$, colitis $(1.7 \%)$, and hepatitis $(1.4 \%){ }^{72}$

Also in I-SPY2, the combination of the anti-PD-L1 mAb durvalumab, the PARP inhibitor olaparib, and paclitaxel followed by doxorubicin+cyclophosphamide as neoadjuvant therapy demonstrated improved pCR rates compared with paclitaxel followed by doxorubicin/cyclophosphamide alone in patients with high-risk HER2stage II or III breast cancer. Based on results from a total of 73 patients, including 21 with TNBC and 52 with HR+ tumors, the durvalumab plus olaparib plus paclitaxel arm graduated 13 months after enrollment had started with $\geq 0.85 \%$ predictive probability of success. Among the 72 patients who completed surgery and were evaluable for pCR, the final predicted probabilities of success in a future phase III study were $81 \%$ for all HER2- cancers (estimated pCR rate 37\%), $80 \%$ for TNBC (estimated pCR rate $47 \%$ ) and $74.5 \%$ for HR+/HER2- patients (estimated pCR rate $28 \%) .{ }^{73}$ For further discussion of PARP inhibition in combination with immunotherapy see the Novel combination strategies and promising future directions section.

Durvalumab was also evaluated in the GeparNuevo trial, which randomized 174 patients with operable TNBC to receive durvalumab or placebo in addition to standard neoadjuvant nab-paclitaxel. The overall pCR rate in the durvalumab arm was $53.4 \%$ (95\% CI $42.5 \%$ to $61.4 \%$ ) compared with $44.2 \%$ (95\% CI $33.5 \%$ to $55.3 \%$ ) in the control arm. Notably, however, benefit with durvalumab was largely observed in the subset of 117 patients that received durvalumab during a run-in window 2 weeks prior to the initiation of chemotherapy, with pCR rates in this cohort of $61 \%$ versus $41.4 \%$ (odds ratio [OR] $=2.22$; 95\% CI 1.06 to 4.64; $\mathrm{p}=0.035$; interaction $\mathrm{p}=0.048$ ). Although characteristics at baseline were generally balanced between treatment arms, patients in the group receiving durvalumab during the window phase were more likely to have stage IIA tumors and nodal involvement before beginning therapy. Due to the Independent Data Monitoring Committee's concern about the delay in starting chemotherapy, the study was amended to eliminate the window-phase after those 117 patients were enrolled. Significantly higher pCR rates were observed among patients with higher levels of stromal TILs in both arms in the complete cohort of patients $(p<0.01)$. Among the patients who received durvalumab in the window phase, changes in the numbers of intratumoral TILs between baseline and after the window phase significantly predicted achieving pCR $(\mathrm{n}=41)$ in both univariate (OR 5.15; 95\% CI 1.10 to 24.05; $\mathrm{p}=0.037$ ) and multivariate regression analyses (OR 9.36; 95\% CI 1.26 to 69.65; $\mathrm{p}=0.029)$. A trend toward increased response rates in PD-L1+ tumors was observed in both arms, with a significant association between pCR rates and PD-L1+ TCs 
for the durvalumab group $(\mathrm{p}=0.045)$ and between $\mathrm{pCR}$ rates and PD-L1+ ICs in the placebo group $(\mathrm{p}=0.040)$, as measured by the VENTANA PD-L1 (SP263) assay. ${ }^{74}$ The findings of the window cohort in GeparNuevo are provocative and raise the question of how best to sequence immunotherapy and chemotherapy.

The NeoTRIPaPDL1 Michelangelo trial was a randomized phase III trial that enrolled 280 patients with previously untreated TNBC and investigated neoadjuvant chemotherapy with carboplatin and nab-paclitaxel for 8 cycles with or without atezolizumab, followed by surgery and adjuvant anthracycline-based treatment. ${ }^{75}$ The primary endpoint was 5-year EFS and secondary endpoints were pCR and safety. The secondary endpoint of pCR rate for the atezolizumab arm was not significantly higher than the control arm, regardless of PD-L1 status (overall study population: $43.5 \%$ vs $40.8 \%$; PD-L1+ disease: $51.9 \%$ vs $48.0 \%$; PD-L1-negative disease: $32.2 \%$ vs $32.3 \%$ ). Follow-up is ongoing for the primary EFS endpoint.

IMpassion031, a double-blind phase III study, randomized 333 patients with previously untreated stage II-III TNBC 1:1 to receive nab-paclitaxel followed by dosedense doxorubicin and cyclophosphamide plus atezolizumab $(n=165)$ or placebo $(n=168)$ followed by surgery. Patients were unblinded after surgery and adjuvant atezolizumab was continued at a fixed dose of $1,200 \mathrm{mg}$ IV every 3 weeks for 11 doses, for a total of approximately 12 months of therapy. At a median follow-up of 20.6 months in the atezolizumab plus chemotherapy group and 19.8 months in the placebo plus chemotherapy group, pCR was observed in 95 patients $(58 \%$; $95 \%$ CI $50 \%$ to $65 \%$ ) in the immunotherapy arm and 69 patients $(41 \%$; $95 \%$ CI $34 \%$ to $49 \%)$ in the placebo plus chemotherapy group (rate difference $17 \%$; $95 \%$ CI $6 \%$ to $27 \%$; one-sided $\mathrm{p}=0.0044$ [significance boundary 0.0184 ]). For patients with PD-L1+ tumors, the pCR rate was $69 \%$ in the atezolizumab arm (53 out of 77 patients; $95 \%$ CI $57 \%$ to $79 \%$ ) compared with $49 \%$ (37 of 75 patients; $95 \%$ CI $38 \%$ to $61 \%$ ) in the placebo arm, for a rate difference of $20 \%$ (95\% CI, $4 \%$ to $35 \%$; one-sided $\mathrm{p}=0.021$ [significance boundary 0.0184$]$ ). Grade 3-4 AEs were balanced between the arms, and treatment-related serious AEs occurred in $37(23 \%)$ and $26(16 \%)$ patients, respectively, for the atezolizumab and placebo groups. ${ }^{77}$ Data from the ongoing GeparDouze trial will further evaluate the addition of immunotherapy to an anthracycline, taxane, and platinum backbone. ${ }^{78}$

Additional ongoing studies include KEYNOTE-756, a global, randomized, double-blind, phase III study, which is investigating pembrolizumab plus chemotherapy as neoadjuvant treatment followed by pembrolizumab plus endocrine therapy as adjuvant treatment for patients with high-risk, early-stage ER+/HER2- breast cancer. ${ }^{79}$ Also, the ongoing CheckMate 7FL trial is evaluating the benefits of adding nivolumab to standard neoadjuvant chemotherapy and to adjuvant endocrine therapy for patients with newly diagnosed high-risk, HR+/HER2- primary breast cancer. $^{80}$

\section{Adjuvant setting}

Limited data are available on the efficacy of immunotherapy for adjuvant treatment of early-stage breast cancer. Although the experimental arm of KEYNOTE-522 included adjuvant pembrolizumab in addition to neoadjuvant chemotherapy and pembrolizumab, it is unclear whether or not the DFS benefit was derived from the neoadjuvant or adjuvant portions, or both. Several ongoing studies are specifically evaluating checkpoint inhibitors either as monotherapy or in combination with chemotherapy as adjuvant treatment.

The ongoing ALEXANDRA/IMpassion030 study is an international, phase III, open-label trial randomizing a total of 2,300 patients with stage I-II TNBC 1:1 to receive standard adjuvant chemotherapy with or without atezolizumab. $^{76}$ The primary endpoint is invasive disease-free survival (iDFS) and secondary endpoints include iDFS by PD-L1 status and lymph node status, OS, safety, and QOL. Patients will be stratified by type of surgery, nodal status, and PD-L1 status.

SWOG S1418 is a randomized phase III trial evaluating pembrolizumab in the adjuvant setting for patients with residual TNBC measuring at least $1 \mathrm{~cm}$ in the breast and/ or lymph node involvement after neoadjuvant chemotherapy and definitive surgery. ${ }^{81} \mathrm{~A}$ total of 1,000 patients are being enrolled and randomized 1:1 to pembrolizumab or observation. The primary endpoint is iDFS, and secondary endpoints are OS, distant relapse-free survival, and safety.

Avelumab is being evaluated as adjuvant therapy in A-Brave, a trial of 335 patients with high-risk TNBC. The protocol-defined patient population will include two strata of patients: those who have completed curative intent surgery of the primary tumor followed by adjuvant chemotherapy with at least 3 cycles of anthracycline and taxane-based therapy (stratum A), and those who have who have completed neoadjuvant chemotherapy with residual disease after curative intent surgery of the primary tumor (stratum B). The primary endpoint is DFS, and secondary endpoints are OS and safety.

\section{Future directions}

Several important questions remain regarding the optimal integration of immunotherapy into treatment for earlystage breast cancer, including the unknown benefits of checkpoint inhibitors in the metastatic setting if patients have previously been treated with these agents for earlystage disease. It will also be key to establish whether pCR corresponds to EFS or OS benefit with ICIs administered in the neoadjuvant setting. Future trials will be needed to address if there is a role for de-escalating the chemotherapy backbone, the role for continuing immunotherapy in patients that do or do not achieve pCR, the optimal duration of immunotherapy, the optimal sequencing of chemotherapy and immunotherapy, and the clinicopathologic features and/or biomarkers that predict who will benefit from the addition of immunotherapy to 
neoadjuvant chemotherapy as well as who is at increased risk for irAEs.

\section{Panel recommendations}

- For all patients with stage II and III TNBC, clinical trial enrollment should be considered if available.

- For patients with stage II and III TNBC, improved pCR rates with either neoadjuvant pembrolizumab or atezolizumab have been observed, regardless of PD-L1 status (LE: 2).

- For patients with high-risk early-stage TNBC, pembrolizumab in combination with chemotherapy as neoadjuvant treatment and then continued as a single agent as adjuvant treatment after surgery is a standard of care based on statistically significant and clinically meaningful improvement in EFS in KEYNOTE-522. Overall survival (OS) data is still maturing (LE: 2).

- For patients with stage II and III TNBC and no available trial, the addition of atezolizumab to standard neoadjuvant chemotherapy may be considered, although not FDA-approved at the time of publication and the IMpassion031 trial was not powered to assess EFS (LE: 2).

- Based on accumulated data to date, immunotherapy regimens for stage II and III TNBC should at least include an anthracycline and a taxane with or without carboplatin (LE: 2).

- For patients with stage II and III TNBC in KEYNOTE-522, patients continued immunotherapy from the neoadjuvant setting into the adjuvant setting. The potential benefits of adjuvant immunotherapy must be weighed against the potential for toxicities with treatment.

\section{DIAGNOSTICS AND BIOMARKER TESTING IN PATIENTS WITH ADVANCED/METASTATIC BREAST CANCER}

Breast cancer is a heterogeneous disease with multiple histologic and molecular subtypes. Generally, breast cancer is classified into three clinically relevant categories: luminal, characterized by expression of ER and/or progesterone receptor (PR); HER2+; and TNBC. Gene expression profiling reveals further distinctions within the IHC-based classifications: luminal A (ER+/HER2-/ Ki67-low) and luminal B (ER+/HER2+ or ER+/HER2-/ Ki67-high), HER2+, claudin-low, and basal-like. ${ }^{82-84}$ TNBC is also heterogeneous and may be classified into six molecular subtypes, ${ }^{85}$ but these classifications are not currently indicated to guide treatment decisions. Luminal disease indicates eligibility for endocrine therapy, and HER2+ tumors are treated with anti-HER2 antibodies including trastuzumab and pertuzumab, antibody-drug conjugates such as trastuzumab deruxtecan, trastuzumab emtansine, or tyrosine kinase inhibitors including neratinib and tucatinib, among other agents. ${ }^{408} 8687$ Guidelines from the College of American Pathologists (CAP) provide more detailed recommendations for scoring HR and HER2 status by IHC, and confirming HER2 IHC equivocal cases by other methods including fluorescence in situ hybridization (FISH) ${ }^{88}{ }^{89}$ Historically, the TNBC subtype lacked targeted therapy options aside from PARP inhibition for BRCA-mutated cancers. ${ }^{90}$ However, patients with TNBC are now eligible for anti-PD-(L) 1 immunotherapy as well as treatment with the Trop-2 directed antibodydrug conjugate sacituzumab govitecan. ${ }^{91}$ At the time of guideline preparation, aside from the pan-tumor antiPD-(L) 1 approvals for TMB-H or MSI-H cancers, TNBC is the only breast cancer subtype for which immunotherapy is approved.

\section{Biomarkers at first relapse}

Genomic instability frequently leads to phenotypic alterations in recurrent tumors compared with the primary site, ${ }^{9293}$ and therefore repeat biopsy of a metastatic lesion is strongly recommended. Treatment may select for modified marker expression in recurrent tumors and genetic alterations that may also contribute to a metastatic tumor's ability to spread. Changes in ER/PR and HER2 expression in metastases have been reported at rates ranging from $30 \%$ to $40 \%$ for ER/PR and $10 \%$ to $15 \%$ for HER2 ${ }^{94}{ }^{95}$ Changes in ER, PR, and HER2 status have also been observed after neoadjuvant chemotherapy, ${ }^{96}$ with implications for therapy selection in recurrent disease. Similarly, metastases frequently harbor distinct genomic alterations compared with primary tumors, leading to the emergence of new actionable mutations in as many as $24 \%$ of patients, including acquired homologous repair deficiency, PI3K mutations, and TMB-H status. 92939798

Notably, PD-L1 expression may be discordant in metastatic versus primary lesions, with higher PD-L1 positivity observed in early-stage lesions relative to metastatic sites. ${ }^{99}$ In general, metastatic tumors contain fewer ICs and decreased markers of immune activation relative to primary breast tumors. ${ }^{1399} 100$ In addition, the degree of immune infiltration and PD-L1 labeling varies between metastatic sites, with certain metastatic niches, such as lung, displaying greater IC and PD-L1 positivity than other immunologically colder niches, such as liver. ${ }^{101}$ Although the liver has classically been referred to as a 'graveyard' for effector T cells and a 'school' for regulatory $\mathrm{T}$ cells, a more nuanced understanding of the roles of several cell types, including monocytes and parenchymal cells, in creating a generally immunosuppressive hepatic microenvironment is beginning to emerge. ${ }^{102} 103$

Importantly, however, PD-L1 IHC as a companion diagnostic for use of atezolizumab or pembrolizumab in TNBC can be performed on either the archival primary tumor tissue or on a metastatic tumor sample. ${ }^{18} 104$ In IMpassion130, any PD-L1+ result, whether in the primary or a metastatic lesion, was associated with clinical benefit. However, the likelihood of a positive PD-L1 result is higher in the primary tumor relative to metastases, and in an inflamed metastatic tumor relative to a non-inflamed tumor. ${ }^{105}$ Thus, it is recommended to pursue biopsy of metastatic lesions, if clinically accessible, for re-assessment of ER/PR/HER2 and additional biomarker analysis 
including next-generation sequencing (NGS) and PD-L1, if appropriate.

\section{Next-generation sequencing}

Genomic sequencing may identify patients who are eligible to receive pembrolizumab based on tumor MSI-H/TMB-H status (for details on the recommended indications see the Tissue-agnostic approvals for checkpoint inhibitors section). Many commercially available NGS assays also offer PD-L1 testing, although none are currently approved as companion diagnostics for immunotherapy in breast cancer. The FoundationOne CDx assay is FDA-approved as a companion diagnostic to identify TMB-H tumors, for which pembrolizumab monotherapy is indicated as an FDA-approved option. ${ }^{40}$ Roughly $5 \%$ of breast cancers overall are TMB-H, ${ }^{46}$ and the rate of hypermutation varies across subtypes, with higher frequencies observed in HER2+ tumors and TNBC. ${ }^{106107}$ Tumor hypermutation may be prognostic for outcomes after treatment with anti-PD-(L) 1 therapies, as a pan-tumor meta-analysis (including breast cancers) found comparable areas under the curve for PD-L1 expression and TMB-H in predicting response. ${ }^{108}$ Increased TMB was also shown to be associated with PFS benefit in IMpassion130, but the association was primarily driven by the PD-L1+ subgroup. ${ }^{47}$ Beyond TMB assessment, NGS is also useful to identify other actionable gene mutations, such PI $3 \mathrm{~K}$ alterations, for which alpelisib in combination with fulvestrant ${ }^{109}$ is a treatment option in ER+/HER2- disease. Amplification of the CD274 locus, which encodes PD-L1, may also be detected by NGS, although the significance is unclear in breast cancer.

Although the FDA approval for use of pembrolizumab in MSI-H tumors does not specify a companion diagnostic, the FoundationOne CDx and other assays include an assessment of MSI. However, the overall prevalence of MSI in breast cancer is low. ${ }^{43-45}$ Although the incidence has yet to be comprehensively elucidated across subtypes, MSI-H has been reported as occurring in less than $2 \%$ of all breast cancers, ${ }^{42}$ with reported rates as low as $0.9 \%$ for TNBC. $^{41}$

In addition to somatic NGS, germ-line genetic testing in the metastatic setting may also guide eligibility for PARP inhibitor therapy among BRCA1 and BRCA2 mutation carriers. ${ }^{110}{ }^{111}$ Defective DNA repair due to mutations in BRCA1 and BRCA2 leads to genomic instability and elevated TMB. ${ }^{112} 113$ However, BRCA1 and BRCA2 play non-overlapping roles in maintaining genomic integrity, ${ }^{114}$ which may underlie the distinctive immunophenotypes that have been associated with mutations in either gene. For example, increased PD-L1 expression and a higher abundance of TILs were reported in BRCA1mutant but not BRCA2-mutant tumors. ${ }^{113}$ Currently, it is unknown whether germ-line BRCA1 or BRCA2 mutations confer additional sensitivity to immunotherapy, or whether an optimal sequencing strategy for immunotherapy with PARP inhibitors exists. Additionally, other mutations in DNA damage response genes may cause tumors to become deficient in homologous recombination repair, a phenotype known as 'BRCA-ness,' that may also be used to predict benefit with PARP inhibition and platinum-based chemotherapy. ${ }^{115} 116$ Notably, in the IMpassion 130 trial, germ-line deleterious $B R C A 1 / 2$ mutations did not predict benefit to atezolizumab plus nabpaclitaxel independently of PD-L1 positivity. ${ }^{23}$ However, combination therapy with anti-PD-1 and PARPi is an active area of investigation (for more details on emerging therapies see the Novel combination strategies and promising future directions section).

\section{PD-L1 expression}

PD-L1 expression in the tumor microenvironment can represent an immunologic brake on antitumor immune responses, as evidenced by PD-L1 expression by ICs, or an immune evasion strategy by the cancer, as evidenced by constitutive or adaptive PD-L1 expression by TCs. ${ }^{117}$ PD-L1 is an important, but imperfect, predictive biomarker for response to PD-(L) 1 checkpoint inhibition across tumor types, ${ }^{118}$ including TNBC. ${ }^{18}{ }^{26}$ The role of PD-L1 as a prognostic biomarker in breast carcinoma has conflicting results in the literature. ${ }^{119}$ Meta-analyses suggest that PD-L1 expression on ICs is a favorable prognostic feature, ${ }^{14}{ }^{120}$ but additional prospective and standardized assessments are warranted. Additionally, PD-L1 'positivity' rates in breast cancer vary widely in the literature, reflective of heterogeneous sample sizes and subtype composition, testing methods, and interpretation criteria. Generally, PD-L1 expression is most common in TNBC and HER2+ breast cancer subtypes, ${ }^{101113117}$ as well as in tumors with high TILs. ${ }^{10} 1113105117$ In TNBC, PD-L1 expression is mostly observed on ICs, and PD-L1 positivity is more common in ICs in primary tumors than in recurrent or metastatic samples. ${ }^{2105}{ }^{121}$ PD-L1 positivity is uncommon in ER+ breast cancer and in non-inflamed tumors.

\section{PD-L1 IHC assays and interpretation}

As of the current writing, four anti-PD-L1 antibody clones are commonly used to evaluate tumor samples via IHC: SP142, SP263, 22C3 and 28-8. Although the individual PD-L1 antibody clones SP142, SP263, 22C3 and 28-8 are equally sensitive for PD-L1, ${ }^{122}$ the associated commercially available assays (ie, testing platforms, components, and methods) have different sensitivities for PD-L1. ${ }^{123-126}$ Characteristics of the four available antibodies and companion assays are summarized in table 5 .

Three PD-L1 assays have been designated as 'companion diagnostics' by the FDA, one of which is indicated for breast cancer: the VENTANA PD-L1 (SP142) assay and the PD-L1 IHC 22C3 pharmDx assay. The companion diagnostic indication for TNBC for the VENTANA PD-L1 (SP142) assay was withdrawn in 2021. The PD-L1 IHC 28-8 pharmDx assay ${ }^{127}$ is not approved for breast cancer. Both the PD-L1 IHC 22C3 pharmDx assay and the PD-L1 IHC 28-8 pharmDx assay are run on the Dako platform. The use of these assays is coupled to the FDA-approved 
Table 5 Summary of anti-PD-L1 antibodies and companion assays

\begin{tabular}{|c|c|c|c|c|c|}
\hline $\begin{array}{l}\text { Antibody } \\
\text { clone }\end{array}$ & Assay & Platform & $\begin{array}{l}\text { PD-L1 scoring for breast } \\
\text { cancer }\end{array}$ & $\begin{array}{l}\text { Companion } \\
\text { diagnostic status }\end{array}$ & $\begin{array}{l}\text { Companion diagnostic } \\
\text { approval for TNBC }\end{array}$ \\
\hline SP142 & $\begin{array}{l}\text { VENTANA PD-L1 } \\
\text { (SP142) }\end{array}$ & VENTANA & $\begin{array}{l}\text { IC score=the percentage of } \\
\text { the tumor area containing ICs } \\
\text { labeling with PD-L1 at any } \\
\text { intensity above background }\end{array}$ & Yes & $\begin{array}{l}\text { IC score } \geq 1 \% \text { indicates } \\
\text { eligibility for atezolizumab } \\
\text { (+nab-paclitaxel) }\end{array}$ \\
\hline $22 \mathrm{C} 3$ & $\begin{array}{l}\text { PD-L1 IHC 22C3 } \\
\text { pharmDx }\end{array}$ & Dako & $\begin{array}{l}\text { CPS=number of PD-L1 } \\
\text { staining cells (including TCs, } \\
\text { lymphocytes, and macrophages), } \\
\text { divided by the total number of } \\
\text { viable TCs, multiplied by } 100\end{array}$ & Yes & $\begin{array}{l}\text { CPS } \geq 10 \text { indicates eligibility } \\
\text { for pembrolizumab } \\
\text { (+chemotherapy) }\end{array}$ \\
\hline $28-8$ & $\begin{array}{l}\text { PD-L1 IHC 28-8 } \\
\text { pharmDx }\end{array}$ & Dako & Not applicable & No & None \\
\hline SP263 & $\begin{array}{l}\text { VENTANA PD-L1 } \\
\text { (SP263) }\end{array}$ & VENTANA & Not applicable & $\begin{array}{l}\text { Not for breast } \\
\text { cancer }\end{array}$ & None \\
\hline
\end{tabular}

CPS, combined positive score; IC, immune cell; IHC, immunohistochemistry; PD-L1, programmed death-ligand 1; TC, tumor cell; TNBC, triple-negative breast cancer.

use of specific anti-PD-(L) 1 checkpoint inhibitors in respective clinical scenarios. ${ }^{117} 1181^{127-129}$ In the US, the FDA-approval for use of ICIs in this setting requires determination of PD-L1 status with a companion diagnostic that is FDA-approved for use in breast cancer ${ }^{104}$.

The scoring criteria to determine PD-L1 status in TNBC with the SP142 assay is the 'IC score', ${ }^{130}$ which is different from the TC score, tumor proportion score, and CPS utilized to assess PD-L1 status with the 22C3 and 28-8 assays. ${ }^{118}$ The IC score is the percentage tumor area occupied by PD-L1+ ICs, including lymphocytes, plasma cells, neutrophils, and macrophages. TNBC is considered 'PD-L1 positive' and the patient eligible to receive atezolizumab per the formerly FDA-approved indication if the tumor shows PD-L1+ ICs occupying $\geq 1 \%$ of the tumor area. The indication for atezolizumab for TNBC was withdrawn in 2021. By contrast, the scoring criteria to determine PD-L1 status in TNBC with the 22C3 assay is the CPS scoring system, which is the total number of PD-L1+ cells (including TCs, lymphocytes, and macrophages) divided by the total number of viable TCs, multiplied by 100. TNBC is considered 'PD-L1 positive' and the patient eligible to receive pembrolizumab per the FDA-approved indication if the tumor has CPS $\geq 10$.

Of note, the label indications for both pembrolizumab and atezolizumab do not explicitly specify which companion diagnostic should be used to assay tumor samples for PD-L1; each merely states that expression should be 'determined by an FDA-approved test'. However, the approvals do differ in eligibility criteria-atezolizumab is indicated for patients whose tumors express PD-L1 as defined by $\mathrm{IC} \geq 1$, whereas pembrolizumab is indicated for patients whose tumors express PD-L1 as defined by $\operatorname{CPS} \geq 10$. Therefore, it is important to use the appropriate assay to measure PD-L1 expression when considering treatment with pembrolizumab (ie, 22C3) or atezolizumab (ie, SP142). This is particularly important as clinical concordance is suboptimal, as demonstrated by a retrospective assay comparison in IMpassion130. ${ }^{131} 132$

There is substantial debate regarding inter-observer variability and reproducibility of PD-L1 scoring in ICs with the SP142 assay, particularly at the 1\% threshold for positivity. ${ }^{133} 134$ The use of pictoral interpretation guides, digital image analysis software, and standardized training may mitigate some of the variability, but additional studies are warranted. ${ }^{135}$ PD-L1 IHC assays and interpretation are also limited by the lack of standardized control of varying expression levels for validation. Implementation of standard control slides for assay validation and laboratory proficiency testing have been proposed to standardize PD-L1 assessment across sites. ${ }^{136}$

The SP142 assay is also less sensitive than the other antibodies with their associated assays (ie, SP263, 22C3, and 28-8 assays), which is reportedly attributable to assay conditions intended to optimize IC labeling. ${ }^{137}$ These assays are not directly interchangeable, and when using a $>1 \%$ threshold for positivity, the latter assays will classify more TNBC as PD-L1+ than the SP142 assay. Post-hoc analysis of the IMpassion130 clinical trial suggests that these additional patients identified as PD-L1+ by the other assays do not demonstrate the same benefit from atezolizumab as the group of patients identified as PD-L1+ by the SP142 assay. ${ }^{131}$ To fully validate the use of the SP263, 22C3, or 28-8 assays to identify the same patient population as demonstrated benefit from atezolizumab in the IMpassion130 clinical trial, the scoring threshold to determine positivity must be adjusted to achieve equal positive predictive value as the SP142 assay.

Of note, multiple clinical trials assessing the use of other PD-(L) 1 ICIs in breast cancer are ongoing 26386374 138-142 (for more details of the studies, see the Emerging data on PD-(L) 1 inhibitors sections in the Advanced breast cancer and Early-stage breast cancer sections). The potential approvals of these agents could be coupled with 
different PD-L1 IHC companion diagnostics, or perhaps not require PD-L1 assessment for eligibility at all. Current data support the importance of PD-L1 expression for patient selection in metastatic disease but not necessarily in early breast cancer. ${ }^{26} 7077143$

\section{PD-L1 specimen considerations}

Patterns of immune infiltration and PD-L1 expression can vary between a primary and metastatic tumor, as well as between different metastatic sites, as discussed above. Assessment of PD-L1 status can be particularly challenging in a tissue sample of metastatic carcinoma involving a lymph node, because PD-L1 expression should be assessed only in the tumor-infiltrating ICs located within the tumor area and not the normal resident ICs of the lymph node. If possible, a non-lymph node tumor section is preferable for PD-L1 assessment. Additionally, as of this guideline preparation, neither the SP142 assay nor the 22C3 assay is validated for use in decalcified specimens or fine needle aspirated tissue smears or cell blocks, ${ }^{130}$ and these specimens should not be used for PD-L1 testing in this setting. Data from IMpassion130 supports using atezolizumab and nab-paclitaxel for patients with metastatic TNBC with any tissue sample, whether metastatic or primary, that is determined to be PD-L1+ by the SP142 assay. As discussed above, metastatic tumors to the liver are less likely to be inflamed and thus less likely to be PD-L1+, with median IC score as low as $0.5 \%$ for liver compared with $3 \%$ in lymph nodes and $1 \%$ in primary breast sites. ${ }^{101}$ If multiple biopsy sites are available, testing for PD-L1 in liver samples should be avoided. However, if no other sites are clinically feasible, despite small numbers, PD-L1+ results from liver biopsies have predicted response to immune checkpoint inhibition. ${ }^{144}$

\section{Predictive value of PD-L1 expression for response to treatment with anti-PD-(L)1 therapy}

Conflicting reports have emerged on the predictive power of PD-L1 expression and response to therapy depending on the treatment setting. In the advanced setting, the treatment effect of immune checkpoint inhibition increased with higher levels of PD-L1 expression in the phase III KEYNOTE-355 study evaluating the addition of pembrolizumab to chemotherapy for previously untreated, locally recurrent, inoperable, or metastatic TNBC. $^{26}$ Similarly, in IMpassion130, pre-specified biomarker analysis found that PD-L1 expression on ICs occupying $\geq 1 \%$ of the tumor area defined a threshold that was strongly predictive of efficacy for atezolizumab plus nab-paclitaxel in the advanced setting. ${ }^{23}$ Conversely, in trials to date for early-stage disease, response rates have generally been consistent across PD-L1+ and PD-L1negative tumors. In IMpassion031, improved pCR rates were seen with the addition of atezolizumab to neoadjuvant chemotherapy in the entire ITT population and no differences were seen associated with PD-L1 status. ${ }^{77}$ Additionally, in KEYNOTE-522, which evaluated the addition of pembrolizumab to neoadjuvant chemotherapy, the benefits with ICI treatment were generally consistent across subgroups regardless of PD-L1 expression. ${ }^{139}$

\section{Other biomarkers}

In breast cancer, TILs are assessed within the confines of the carcinoma and are defined as the percentage stromal area occupied by mononuclear inflammatory cells (ie, stromal TILs [sTILs]). ${ }^{145}$ Assessment of TILs is included as a prognostic biomarker in the 2019 WHO classification of Breast Tumors. ${ }^{146}{ }^{147}$ Although consensus guidelines have been published ${ }^{145}$ that have supported retrospective analyses and the incorporation of TIL evaluation as integral and integrated biomarkers in several trials (see www. TILsinBreastCancer.org), TIL scores are not indicated for routine clinical practice. Concerns about inter-observer variability in TIL assessment have also been raised, ${ }^{148}$ but machine-learning-based approaches for scoring may improve reproducibility in the future. sTILs have demonstrated predictive prognostic power in TNBC and HER2+ breast cancer, with higher levels of infiltration being linked to improved outcomes. ${ }^{12} 1455^{149-151}$ In a 2009 study that investigated the relationship between lymphocytic infiltration at diagnosis in node-positive samples with clinical outcomes from the BIG 01-98 adjuvant phase III trial, a $10 \%$ increase in sTILs was associated with $15 \%$ reduced risk of relapse $(\mathrm{p}=0.025)$ and $17 \%$ reduced risk of death in TNBC. For HER2+ tumors treated with anthracyclineonly chemotherapy, a significant interaction was observed between increasing sTILs and both DFS and OS (DFS $\mathrm{p}=0.042$; OS $\mathrm{p}=0.018) .{ }^{151}$ For ER+/HER2- breast cancer, the prognostic value is less clear. One pooled analysis of 3,771 patients receiving neoadjuvant therapy demonstrated that although increases in sTILs were associated with response to chemotherapy in all molecular subtypes, TILs were only correlated with longer OS in TNBCno association with survival was shown in HER2+ breast cancer, and increased TILs was linked to shorter OS in luminal-HER2- disease. ${ }^{152}$ Additionally, a case-cohort series of 987 patients with early ER+/HER2- breast cancer found that high TILs was associated with better distant DFS, but only in the group of patients treated with adjuvant therapy. ${ }^{151} 153$ In contrast to the data in early breast cancer, exploratory analyses of IMpassion 130 showed that sTILs were associated with PD-L1+ status but did not independently predict PFS nor OS in advanced TNBC. ${ }^{23}$

The functional characteristics and spatial distribution of TILs within the tumor microenvironment may be important in the generation of effective antitumor immune responses. While multiplex, high-resolution TIL profiling technologies are, at the time of writing, purely investigational, an increased understanding of the prognostic value of individual infiltrating T-cell subsets may inform the development of future biomarkers or rationally designed immunotherapeutic approaches. Substantial heterogeneity may exist in the spatial distribution of TILs between regions of samples taken from the same tumor, ${ }^{154}$ although the average lymphocyte score from a single biopsy has been shown to reasonably represent the 
tumor as a whole. ${ }^{155}$ In TNBC, enrichment of CD8 ${ }^{+}$TILs with a characteristic tissue-resident memory gene expression signature was significantly associated with improved patient survival in early-stage disease, having greater prognostic power than CD8 expression alone. ${ }^{156}$ In ER+ breast cancer, TIL spatial heterogeneity was more highly prognostic for late recurrence 5 years after endocrine therapy than any other tumor immune score measures. ${ }^{157}$

Other biomarkers may also predict response to immune checkpoint blockade. The genomic amplification of $C D 274$, the gene that encodes PD-L1, is frequently observed in Hodgkin lymphoma and sometimes detected in solid tumors including breast cancers. ${ }^{158}{ }^{159}$ Amplifications of $C D 274$ may have important prognostic value for response to ICI therapy. One analysis of 118,187 tumor samples (including a subset of 2,039 samples with clinical annotation) found an overall prevalence of CD274 amplification of $0.7 \%$ overall, and just $0.02 \%$ in breast cancers. Importantly, the ORR for ICI-treated patients with solid tumors with CD274 amplification was $66.7 \%$, with median PFS of 15.2 months. ${ }^{159}$ In TNBC specifically, increased copy number for the chromosomal region that encodes PD-L1, PD-L2, and JAK2-sometimes called the PDJ locus, 9p24-is more frequently detected in TNBC compared with in ER+ and HER2+ subtypes and in other solid tumors. ${ }^{160}$ Exposure to neoadjuvant chemotherapy may select for 9p24 amplifications in TNBC, ${ }^{161}$ and amplifications at the locus correlate with increased protein expression of PD-L1 by IHC and mRNA in situ hybridization. ${ }^{158}$ Amplification of 9p24 was associated with worse OS in studies of patients not receiving immunotherapy, ${ }^{160161}$ and studies are ongoing to determine prognostic value of this biomarker in the context of immune checkpoint inhibition. Exploratory analysis of 126 patients with metastatic breast cancer who were enrolled in the SAFIR-IMMUNO study (a randomized trial comparing durvalumab to maintenance chemotherapy) found that $20 \%$ of all tumors and $35 \%$ of TNBCs had copy gain (3 or 4 copies) or amplification ( $>4$ copies) of CD274. Notably, only patients with amplifications in $C D 247$ had improved OS with durvalumab (HR $0.18 ; 95 \%$ CI 0.05 to 0.71 ). ${ }^{162}$

The identification of additional biomarkers for patient selection and response prediction for immunotherapy is an ongoing area of research. Promising areas include mRNA-based signatures, such as an interferon gene expression profile that has been shown to predict response to PD-1 blockade in a variety of tumor types, including breast cancer, ${ }^{163}$ and liquid biopsy-based biomarkers that quantify circulating TCs, nucleic acids, and proteins. ${ }^{164}$ Future prospective trials will be needed to validate any novel immunotherapy biomarkers and ensure generalizability across breast cancer subtypes and patient populations.

\section{Panel recommendations}

- For patients with TNBC being considered for treatment with pembrolizumab in combination with chemotherapy, tumor tissue should be tested for
PD-L1 by the PD-L1 22C3 pharmDx assay and scored by the CPS system, until PD-L1 assays are harmonized (LE: 2). A TNBC is PD-L1+ by 22C3, and the patient eligible for pembrolizumab, with a CPS $\geq 10$.

- PD-L1 testing is not recommended for patients with early-stage breast cancer at this time (LE: 2).

- Although PD-L1 testing of primary lesions may not correlate with expression in metastatic disease, benefit was observed in IMpassion130 with any PD-L1+ result regardless of whether primary or metastatic tumor. PD-L1 testing should be performed on the metastatic tumor, if available, but testing on primary tumor is acceptable (LE: 2).

- When considering metastatic sites to test for PD-L1, it is preferable to prioritize extrahepatic sites or the primary tumor, if available.

- PD-L1 testing should not be performed on fine needle aspirated cell-block specimens or decalcified bone.

- Stromal TIL assessment in primary lesions is prognostic in early TNBC and HER2+ breast cancer (LE: 1), but has not been validated to direct clinical decisionmaking for chemotherapy or immunotherapy.

- Biomarker assessment, including repeat receptor profiles (ER/PR/HER2) and PD-L1 status as well as NGS should be considered at first relapse (LE: 3).

\section{EVALUATION AND MANAGEMENT OF TREATMENT RESPONSE Imaging}

As with cytotoxic therapy, recommended evaluation of tumor response to immunotherapy should be performed with a CT scan or MRI. The WHO criteria ${ }^{165}$ and the Response Evaluation Criteria In Solid Tumors (RECIST) guidelines ${ }^{166}$ define standard measurement methods for converting radiology image observations into quantitative and statistically tractable frameworks for measuring changes in tumor size associated with therapy. However, the unique patterns of responses observed with immunotherapy have led to the development of several modified criteria for reporting responses in solid tumors (described below). According to some analyses, conventional response criteria may underestimate ORR for immunotherapytreated patients by as much as $15 \% .{ }^{167}$ Additionally, due to the inability to differentiate metabolically active cancer cells from activated ICs and inflammation, standard positron emission tomography (PET) imaging may present challenges in assessing tumor response for patients with breast cancer on immunotherapy. ${ }^{168}$

The mechanism of action of ICIs can cause unusual response patterns on imaging when compared with traditional responses to cytotoxic chemotherapy. While many patients still may have an initial reduction in tumor burden, other patients may experience non-traditional responses like initial tumor growth followed by tumor reduction (termed pseudoprogression), a period of rapid tumor growth (termed hyperprogression), or a period of prolonged stability followed by an eventual decrease in tumor growth. ${ }^{169}$ Although pseudoprogression, an apparent increase in tumor burden or appearance of new 
Table 6 Comparison of immune-related response criteria (Adapted from Kataoka \& Hirano, Ann Trans/ Med, 2018) ${ }^{288}$ )

\begin{tabular}{|c|c|c|c|c|c|}
\hline & RECIST v1.1 ${ }^{289 *}$ & $\operatorname{irRC}^{171}$ & irRECIST $^{172}$ & iRECIST $^{174}$ & imRECIST $^{173}$ \\
\hline Based on & RECIST & WHO criteria ${ }^{165}$ & $\begin{array}{l}\text { irRC and RECIST } \\
\text { v1.1 }\end{array}$ & RECIST v1.1 & irRC and RECIST v1.1 \\
\hline $\begin{array}{l}\text { Definition of } \\
\text { PD }\end{array}$ & $\begin{array}{l}20 \% \text { increase from } \\
\text { nadir and } 5 \mathrm{~mm} \\
\text { absolute increase } \\
\text { in the sum of target } \\
\text { lesions }\end{array}$ & $\begin{array}{l}25 \% \text { increase from } \\
\text { nadir }\end{array}$ & $\begin{array}{l}20 \% \text { increase from } \\
\text { nadir }\end{array}$ & $\begin{array}{l}20 \% \text { increase from } \\
\text { nadir (confirmation } \\
\text { necessary) }\end{array}$ & $\begin{array}{l}20 \% \text { increase from } \\
\text { nadir }\end{array}$ \\
\hline New lesions & Do define PD & Do not define PD & Do not define PD & $\begin{array}{l}\text { Do not define PD } \\
\text { Not included in sum } \\
\text { of measurements }\end{array}$ & $\begin{array}{l}\text { Do not define PD } \\
\text { Included in sum of } \\
\text { measurements }\end{array}$ \\
\hline
\end{tabular}

mcluded in sum of Included in sum of Included in sum of

\begin{tabular}{|c|c|c|c|c|c|}
\hline Confirmation & $\begin{array}{l}\text { Protocol-specific } \\
\text { based on the } \\
\text { therapy, the } \\
\text { disease, the } \\
\text { anticipated time } \\
\text { to response and } \\
\text { progression as well } \\
\text { as cost and patient } \\
\text { convenience }\end{array}$ & 4 weeks & 4 weeks & $\begin{array}{l}4 \text { weeks }- \text { not longer } \\
\text { than } 8 \text { weeks }\end{array}$ & 4 weeks \\
\hline $\begin{array}{l}\text { Outcomes in } \\
\text { development } \\
\text { cohort }\end{array}$ & $\begin{array}{l}\text { Prospective cohort } \\
\text { with data from } \\
>6,500 \text { patients, } \\
\text { simulation studies, } \\
\text { and literature } \\
\text { reviews }\end{array}$ & $\begin{array}{l}\text { OS in ipilimumab- } \\
\text { treated melanoma }\end{array}$ & $\begin{array}{l}\text { irRC response } \\
\text { in advanced } \\
\text { ipilimumab-treated } \\
\text { melanoma }\end{array}$ & Consensus-based & $\begin{array}{l}\text { OS in atezolizumab- } \\
\text { treated advanced } \\
\text { NSCLC and mUC }\end{array}$ \\
\hline
\end{tabular}

${ }^{*}$ RECIST v1.1 was used in KEYNOTE-119, KEYNOTE-355, and IMpassion130.

imRECIST, immune-modified RECIST; iRECIST, immunotherapy RECIST; irRC, immune-related response criteria; irRECIST, immune-related RECIST; mUC, metastatic urothelial cancer; NSCLC, non-small cell lung cancer; OS, overall survival; PD, progressive disease; ; RECIST, Response Evaluation Criteria In Solid Tumors; WHO, World Health Organization.

lesions on therapy, has been described in up to roughly $10 \%$ of patients with melanoma treated with checkpoint inhibitors, ${ }^{170}$ the phenomenon seems to be tumordependent with much lower rates of pseudoprogression $(<5 \%)$ noted in breast cancer studies. ${ }^{63} 144$ Potential mechanisms behind the appearance of enlarged lesions in solid tumors on imaging include infiltration of activated $\mathrm{T}$ cells, an inflammatory response due to cytokine release, or may simply reflect the time needed for the immune system to mount an appropriate response for tumor control. ${ }^{169}$

Because the traditional RECIST system does not consistently capture clinical benefit with immunotherapy, several systems, including immune-related response criteria (irRC), ${ }^{171}$ the immune-related RECIST (irRECIST), ${ }^{172}$ immune-modified RECIST, ${ }^{173}$ and immunotherapy RECIST (iRECIST) ${ }^{174}$ were developed (table 6). Evaluation of tumor burden in two dimensions is mandated by irRC, which requires more effort than the one-dimensional evaluation of RECIST. ${ }^{175}$ The irRECIST criteria combine the features of irRC and RECIST and requires only one-dimensional measurement ${ }^{172}$; however,
irRECIST has not been consistently applied across studies and therefore may not permit cross-study comparisons of efficacy. In contrast to irRECIST, measurements of the new lesion(s) are not incorporated into the tumor burden with iRECIST. Additionally, iRECIST was developed by consensus.

A retrospective analysis of 14 published trials found that responses assessed by iRECIST compared with RECIST v1.1 led to a roughly 1 month longer median DOR in the analysis population, ${ }^{176}$ a modest but potentially meaningful difference. Ongoing efforts are attempting to develop a standardized, universal scoring system for pathologic response to PD-(L) 1 blockade (immunerelated pathologic response criteria) that encompasses features characteristic of immunotherapy for diverse tumor types, ${ }^{177} 178$ but this pan-tumor criteria has not yet been validated in prospective studies. Additionally, an immunotherapy response assessment for neuro-oncology, iRANO,${ }^{179}$ has been developed, which may have future implications for the management of brain metastases, though these criteria have not been extensively validated in patients with breast cancer. Notably the randomized 
phase III studies completed to date in locally advanced/ metastatic TNBC (KEYNOTE-119, KEYNOTE-355, and IMpassion130) adopted RECIST v1.1 for determination of disease status. Currently it is recommended that if one of the immune response criteria is used, the standard RECIST measurements should also be used to help with validity and cross trial comparisons.

\section{Treatment beyond progression}

For several decades, the overarching dogma for managing recurrent or refractory disease was that therapy should be changed at progression, based on the assumption that resistance is stable. With a growing body of evidence for the existence of unstable mechanisms of drug resistance,${ }^{180}$ however, chemotherapy rechallenge has become an established paradigm across several disease settings. In breast cancer, retreatment with the same chemotherapy regimen that was used as adjuvant therapy has demonstrated clinical benefit, ${ }^{181} 182$ provided there is a sufficient disease-free interval. In the HER2+ setting, treatment with trastuzumab beyond progression has been shown to increase clinical benefit rate for patients receiving capecitabine $^{183}$ and increases OS in patients with brain metastases. ${ }^{184}$ Whether treatment beyond progression offers similar benefits for patients receiving immunotherapy remains an ongoing area of investigation.

Based on current iRECIST guidelines, as well as the Trial Reporting in Immuno-Oncology guidelines published in 2018 by the American Society of Clinical Oncology (ASCO) and SITC, ${ }^{185}$ clinical assessment and patient functional status are important when determining if a patient should continue on a given immunotherapy in the setting of progressive disease. Both guidelines specify that for patients to receive treatment beyond progression, the patient should have stable or improved clinical condition, have no severe laboratory abnormalities, and be tolerating the treatment well with limited/mild side effects. Most importantly, there should be no clinical progression and no additional progression noted on subsequent confirmation imaging scans.

\section{Management of isolated sites of progression on immunotherapy}

The appearance of new lesions while on treatment in the metastatic setting, including immunotherapy, is not necessarily a reason to discontinue therapy. As mentioned above, pseudoprogression may result in the appearance of new lesions which then later decrease in size on subsequent reimaging. There may also be true progression with the appearance of a new solitary lesion (ie, oligoprogression). This can occur due to tumor heterogeneity and/or the development of new resistance mechanisms to therapy. In these cases, there may be an isolated site of disease progression in patients who otherwise have a good response to treatment. There is no standard management for these isolated lesions in the setting of otherwise responsive disease. ${ }^{174}$
Once a new lesion(s) is confirmed, is it reasonable to consider local treatment(s) to the isolated site(s) of progression, as long as the patient has good performance status and is otherwise responding to or stable on the current treatment. Localized treatment may involve local ablative therapies like brachytherapy or stereotactic body radiation therapy (SBRT) and/or surgical therapies like a metastasectomy, as is currently done in many other metastatic tumor types. Although studies are limited in the breast cancer disease setting, improved outcomes after surgical resection of isolated sites of progression have been reported for gastrointestinal, adrenal, and large single brain metastases for patients with melanoma being treated with immunotherapy. ${ }^{186-188}$ In breast cancer, a systematic review of outcomes after local ablative therapies for the management of hepatic metastases reported CR rates of roughly $90 \%$ after hepatic resection, stereotactic radiofrequency ablation, SBRT, and brachytherapy. ${ }^{189}$

In cases where surgery is not an option, radiation therapy may synergize with immunotherapy through the induction of immunogenic cell death. ${ }^{190}$ Until recently, the evidence for efficacy was limited to case reports and preclinical models. A small, signal-seeking phase II trial establishing the safety and early activity of pembrolizumab with radiation in heavily pre-treated, metastatic TNBC regardless of PD-L1 status demonstrated tolerabilty and a signal of activity with the combination. ${ }^{191}$ At this time, the benefit of local treatment for an isolated progressive tumor is still being determined. However, given that radiation is a commonly accessible local treatment that is already well-integrated into cancer treatment, many physicians will opt for local therapy in order to continue on a treatment regimen that is controlling other sites of disease.

\section{Duration of immunotherapy}

The decision to discontinue therapy is a challenging and ongoing topic of debate in the immuno-oncology field. Although the ultimate endpoint for any cancer treatment is overall survival, ongoing and durable responses have been seen with immunotherapy, raising the difficult question of when it is appropriate to stop therapy. Of those patients who have durable responses to immunotherapy, it is not known how long treatment should continue. Experience from melanoma suggests that durable remissions may be maintained in as many as $85 \%$ of patients who stop receiving anti-PD-1 therapy after achieving CR. ${ }^{186} 192$ At this time, there are also accounts of patients with breast cancer who have come off immunotherapy due to toxicity but continue to have durable responses years after treatment was stopped. ${ }^{193}$ Most initial trials of ICIs for breast cancer had a set period of treatment, but at this time as these agents matriculate into routine oncology practice, the ideal duration of therapy is unknown.

\section{Post-immunotherapy treatment choice}

Limited data are available on which to base a decision on optimal treatment after immunotherapy. In the 
IMpassion130 trial, subsequent anticancer therapy was administered to 242 patients (53.7\%) in the atezolizumab plus nab-paclitaxel group and to 272 patients $(60.3 \%)$ in the placebo plus nab-paclitaxel group, with most patients receiving chemotherapy during follow-up and less than $4 \%$ receiving further immunotherapy. ${ }^{18}$ Standard chemotherapy agents have immunomodulatory effects, ${ }^{194}$ however, the effects of cytotoxic agents are pleiotropic and potential interactions with immunotherapies are difficult to predict from pharmacokinetics and preclinical models alone. While optimal sequencing is unknown at this time, understanding effects of various treatments on the tumor microenvironment may help to guide future studies on optimal sequencing of therapies.

\section{Panel recommendations}

- The application of formal response criteria (ie, RECIST) are not currently recommended off-study. If one of the immune response criteria is used, the standard RECIST measurements should also be used to help with validity and cross-trial comparisons.

- When pseudoprogression is suspected and treatment beyond progression is being considered, the patient should have stable or improved clinical condition, no severe laboratory abnormalities, and be tolerating the treatment well with limited/mild side effects. Treatment beyond progression should be discontinued in cases where clinical progression occurs or if additional progression is confirmed on subsequent imaging scans.

- For management of isolated site(s) of progression for a patient receiving immunotherapy, it is reasonable to consider local therapy for the isolated site(s) of progression as long as the patient has good performance status and is otherwise responding to the current treatment. However, there are no data that local treatment will improve clinical outcomes.

\section{TOXICITY CONSIDERATIONS: PATIENT SELECTION AND MANAGEMENT}

\section{Baseline factors for consideration}

The decision to proceed with immunotherapy depends on the likelihood that the tumor will respond to treatment and the patient's projected ability to tolerate therapy. For patients with early-stage disease, the potential for benefit with immunotherapy must also be weighed against the risk of irAEs. Patients with autoimmune disorders, chronic viral infections, AIDS, ongoing clinically significant immunosuppressant use, organ dysfunction, pregnancy, older age, and impaired functional status are generally considered to be challenging populations to treat with checkpoint inhibitors. As more patients receive checkpoint inhibitors in real-world settings, however, emerging data are painting a clearer picture of the risk/ benefit tradeoffs in some groups of patients, and it is becoming clear that some of these "challenging populations' may safely receive treatment.
Patients living with HIV infection have historically been excluded from clinical trials of checkpoint inhibitors due to concerns about potential risk for viral reactivation or increased toxicity on therapy. Before the advent of highly active antiretroviral therapy (HAART), it was believed that the incidence of breast cancer may be lower in patients with AIDS than in the general population. ${ }^{195}$ However, subsequent analyses have revealed that patients living with HIV are at increased risk of mortality for all non-AIDS-related cancers, including breast cancer. ${ }^{196-198}$ Although experience with immunotherapy, specifically in patients with breast cancer and HIV, is limited, a systematic review of ICI treatment in 72 patients with advancedstage cancers who were also being treated with HAART for HIV infection found no new safety signals, comparable response rates to non-HIV-infected individuals, and maintenance of viral suppression as well as $\mathrm{CD} 4^{+} \mathrm{T}$ cell counts. ${ }^{199}$ Prospective trials have also demonstrated safety and efficacy for ICI therapy for patients living with HIV and a variety of solid tumors. ${ }^{200}{ }^{201}$ Although further studies are needed in the breast cancer setting, none of the data to date indicate that HIV infection is an absolute contraindication to ICI therapy, provided the patient is compliant with appropriate antiretroviral therapy.

Some studies have suggested that patients with preexisting autoimmune disorders may be safely treated with anti-PD-(L) 1 therapies. In one study, 52 patients with melanoma and pre-existing autoimmune disorders were treated with either pembrolizumab or nivolumab. Twenty patients (38\%) had flares of their autoimmune disease requiring immunosuppression, but toxicities were generally mild, manageable, and did not necessitate discontinuation of therapy. ${ }^{202}$ The decision to proceed with immunotherapy in a patient with pre-existing autoimmune conditions should include assessing the risk of severe morbidity and/or mortality from the underlying disorder versus risk of relapse or death from the cancer diagnosis. Similarly, if considering using immunotherapy to treat patients with breast cancer receiving immunosuppressive therapy for an underlying autoimmune disorder, it is critical to consider the underlying reason for the immunosuppression as well as the clinical benefit expected from the immunosuppressive agent.

Analyses of outcomes among older patients receiving checkpoint inhibitors in clinical trials ${ }^{203}$ and real-world settings $^{204}$ suggest that the toxicity profiles and response rates for immunotherapy in the elderly do not differ markedly from those in the general population. Those results must be interpreted with a note of caution, however, as the older patients that were included were all relatively fit (ie, good baseline performance status). Geriatric assessments for elderly individuals may be useful to evaluate the potential safety of more intense therapeutic regimens including immunotherapy. ${ }^{205}$

Baseline corticosteroid use has been associated with worse survival outcomes in patients with lung cancer treated with anti-PD-(L) 1 agents. ${ }^{206} 207$ The interpretation of these findings is complicated however, because patients 
receiving corticosteroids for palliative indications may also have significant comorbidities at baseline. One study that analyzed outcomes stratified by reason for corticosteroid administration found that median PFS and OS were significantly shorter among patients who received $\geq 10 \mathrm{mg}$ prednisone for palliative indications associated with their underlying malignancy compared with those who received $\geq 10 \mathrm{mg}$ prednisone for non-cancer related reasons and compared with patients receiving 0 to $<10 \mathrm{mg}$ of prednisone. ${ }^{208}$ The role of glucocorticoids in breast cancer is complex and further complicated due to natural variability in endogenous hormone levels throughout a woman's menstrual cycle. ${ }^{209}$ Glucocorticoid receptor signaling has been linked to the emergence of chemoresistance in breast cancer. ${ }^{210}$ Although scant data are available on the impact of steroid administration on immunotherapy efficacy in the treatment of breast cancer, a meta-analysis including 16 studies of patients with lung cancer demonstrated no adverse effects on survival outcomes when corticosteroids were used for the management of irAEs. ${ }^{211}$

The incidence of breast cancer in solid organ transplant recipients is similar to that in the general population, and given the overall frequency of transplant patients with excellent outcomes, it is likely that providers will encounter patients with breast cancer and a history of solid organ transplantation. ${ }^{212}$ There is limited data on the outcomes of transplant patients with checkpoint inhibitor therapy, but existing data suggests a significant risk of graft rejection. ${ }^{213}$ For this reason, routine ICI treatment of patients with breast cancer and a prior organ transplant outside of a study or at a specialized center is discouraged.

\section{Monitoring patients on treatment (toxicities, time frame)}

The goal of appropriate monitoring during immunotherapy treatment is to promptly detect immune-related toxicities and intervene before these toxicities cause significant morbidity or mortality. An important principle is to properly educate patients and staff about the symptoms that require prompt reporting to avoid life-threatening complications (described in more detail in the Patient education and QOL section). The most frequently reported irAEs in breast cancer ICI trials are rash and pruritus (up to $18 \%$ ), thyroid disorders (up to $12 \%$ ), and liver function abnormalities (up to $10 \%$ ). ${ }^{214}$ Incidences of irAEs reported in trials of ICI monotherapy or combination regimens for TNBC are summarized in table 7 . Particular attention should be paid to new or worsening fatigue, headaches, rash, respiratory symptoms, changes in bowel function, visual changes/eye pain, or musculoskeletal symptoms. Careful monitoring of laboratory studies is also required, including electrolytes, creatinine, glucose, liver function, and thyroid hormone levels.

Baseline history/physical exam should include assessment of autoimmune, infectious, neurologic, bowel, musculoskeletal, or endocrine pre-existing conditions. Pulse oximetry assessment and monitoring is recommended. An electrocardiogram (EKG) should be considered to provide a pre-treatment baseline for comparison with future EKGs obtained due to cardiac-related symptoms. Baseline troponin levels should also be obtained to provide useful information for evaluating potential future cardiotoxicity, and subsequently measured as clinically indicated.

Most immunotherapy agents include monitoring recommendations as part of their prescribing information; for example, the package insert for pembrolizumab recommends monitoring for changes in hepatic and thyroid function. ${ }^{19}$ However, accumulated experience with ICIs in real-world settings has led to the emergence of some general principles on the type and frequency of monitoring needed. Routine monitoring of patients is generally more frequent during the initial 4 cycles of treatment, with clinical assessments and laboratory testing complete blood count (CBC), comprehensive metabolic panel (CMP), hemoglobin A1c (HgbA1c), thyroid stimulating

Table 7 Reported incidence of irAEs in published ICl clinical trials for metastatic TNBC (adapted from D'Abreo and Adams, Nat Rev Clin Oncol, $2019^{214}$ )

\begin{tabular}{|c|c|c|c|c|}
\hline irAE & & All grades (\%) & Grade 3-4 (\%) & Grade 5 (\%) \\
\hline Dermatologic & Pruritus, rash & 18 & 0.5 & 0 \\
\hline \multirow[t]{2}{*}{ Endocrine } & Hypothyroidism & 12 & 0 & 0 \\
\hline & Hyperthyroidism & 5 & 0.1 & 0 \\
\hline \multirow[t]{2}{*}{ Gastro-intestinal } & Hepatitis, elevated transaminases & 10 & 3 & 0.2 \\
\hline & Colitis, diarrhea & 2.5 & 0.45 & 0 \\
\hline Hematologic & $\begin{array}{l}\text { Prespecified autoimmune anemia, lymphopenia, } \\
\text { thrombocytopenia and clotting abnormalities }\end{array}$ & 4 & 1 & 0.2 \\
\hline Respiratory & Pneumonitis & 3 & 0.5 & 0.1 \\
\hline Other $(<1 \%)$ & $\begin{array}{l}\text { Adrenal insufficiency, type } 1 \text { diabetes, ocular, } \\
\text { myocarditis, neurological/meningitis, nephritis/ } \\
\text { elevated creatinine }\end{array}$ & $<1$ & $<0.5$ & 0 \\
\hline
\end{tabular}

$\mathrm{ICI}$, immune checkpoint inhibitor; irAEs, immune-related adverse events; TNBC, triple-negative breast cancer. 
hormone (TSH), free T4 (FT4), and morning serum cortisol recommended at baseline and every 4 weeks. ${ }^{215}$ After the first four cycles then testing intervals can be increased to every 6-12 weeks, or as indicated. Additionally, morning serum cortisol should be measured prior to surgery in patients receiving pembrolizumab in the neoadjuvant setting. Combination immunotherapy regimens may require closer monitoring as immune-related toxicity rates tend to be higher compared with monotherapy. ${ }^{215216}$ Other tests such as amylase, lipase, C-reactive protein, creatine phosphokinase (CPK), erythrocyte sedimentation rate (ESR), brain MRI, CT scans of the thorax/abdomen, pulmonary function tests, troponin and EKGs should be performed as indicated. Most patients with metastatic breast cancer receive some of these imaging tests routinely for assessment of treatment response, so additional scans in asymptomatic patients are generally not required.

Any organ system in the body can be affected by irAEs, and the most commonly reported toxicities across all cancer types are dermatologic, gastrointestinal, endocrine, respiratory, and hepatic ${ }^{215-218}$ For breast cancer, the top three most commonly reported irAEs in published trials are hypothyroidism, rash or pruritus, and hepatitis (see table 7). Although the timing of onset and organ systems affected by irAEs may vary, dermatologic toxicities are among the most frequently reported. In one pooled analysis of patients receiving immunotherapy for metastatic melanoma, skin toxicities tended to occur earlier (median 5 week onset), whereas endocrinopathies and renal toxicities tended to occur later (median 10-15 weeks). The median time to onset for most other immune-related events was about 8 weeks. ${ }^{219}$ For TNBC, the timing to onset for irAEs was similar to that seen in other tumor types. ${ }^{214}$

\section{Management of irAEs}

ICIs are associated with distinct toxicity profiles compared with conventional breast cancer treatments. Oftentimes, the same mechanisms that give rise to antitumor effects also underlie the AEs seen with immunotherapy-namely, uninhibited immune activity. For ICI therapy, the overall incidence of irAEs across published trials has been estimated to be up to $75 \%$ for anti-cytotoxic T lymphocyte antigen-4 (CTLA-4) monotherapy (ipilimumab) and up to $30 \%$ for anti-PD-(L) 1 agents. ${ }^{215}$ Detailed guidelines on the recognition and management of irAEs have been published elsewhere, including by SITC ${ }^{215217} 220$ and care typically includes withholding immunotherapy, administering corticosteroids, and, in some instances, administering second-line immune-modulatory agents such as tumor necrosis factor (TNF) inhibitors. In general, immunotherapy may be continued in the setting of grade 1-2 immune toxicity that can be managed with topical or systemic low-dose steroids, whereas grade $\geq 3$ toxicity, or symptomatic grade 2 toxicity necessitates at least temporary discontinuation of therapy and referral to or consultation with appropriate specialists.

With more data becoming available on the use of immunotherapy in clinical trial and real-world settings, patterns of irAEs that were not evident during individual studies are emerging, such as high rates of thyroiditis. A meta-analysis including 38 randomized clinical trials evaluating the usage of ICIs for treatment of advanced solid tumors including a total of 7,551 patients found an overall incidence of hypothyroidism of $6.6 \% \quad(95 \%$ CI $5.5 \%$ to $7.8 \%){ }^{221}$ The incidence of hypothyroidism in real-world settings may be higher than in clinical trials, as one retrospective analysis of electronic health records for 1,146 individuals treated with ICIs at a single center between 2012 and 2018 identified thyroid irAEs in 19\% of patients. ${ }^{222}$ Another analysis of 29,294,336 records spanning 5 years from the FDA AEs reporting system found that the four most common endocrine-related AEs with ICIs were hypothyroidism, primary adrenal insufficiency, hypophysitis (secondary adrenal insufficiency), and hyperthyroidism. ${ }^{223}$

\section{Patterns of irAEs in patients with breast cancer}

In the IMpassion130 trial, a total of 259 patients (57.3\%) in the atezolizumab plus nab-paclitaxel group and 183 $(41.8 \%)$ in the placebo plus nab-paclitaxel group had AEs of special interest that were suggestive of potential immune-related causes. ${ }^{18}$ In IMpassion130, the leading cause for atezolizumab discontinuation was peripheral neuropathy. Serious AEs were reported in 105 of 453 patients in the atezolizumab group (23\%) compared with 81 of 437 patients in the placebo group (19\%), and one death due to an $\mathrm{AE}$ of special interest was reported in the primary analysis in each arm of the study-in both cases, hepatitis. On secondary analysis, the AEs of special interest that differed substantially between the atezolizumab group and the placebo group were any-grade rash, hypothyroidism, hyperthyroidism, pneumonitis, and adrenal insufficiency. ${ }^{24}$ In KEYNOTE-355, irAEs occurred in $26 \%$ of patients in the pembrolizumab plus chemotherapy arm, with $5 \%$ of patients experiencing irAEs of grade $\geq 3$. The only irAE of grade $\geq 3$ that occurred in 10 or more patients was skin toxicity $(n=10 ; 2 \%)$. In the immunotherapy group, hypothyroidism and hyperthyroidism occurred in $87(15 \%)$ and $15(5 \%)$ patients, respectively. Hypothyroidism and hyperthyroidism occurred in $9(3 \%)$ and $3(1 \%)$ patients in the control group. ${ }^{26}$ Management of immune toxicities in other organ systems follows similar recommendations to existing expert panel guidelines. ${ }^{215217220}$ Referral to appropriate specialists for persistent autoimmunity is recommended.

\section{Late irAEs in immunotherapy-experienced patients}

Unlike the AEs associated with chemotherapies or targeted drugs, irAEs may arise months or even years after cessation of immunotherapy. Definitive data on the incidence rates and severity of delayed onset AEs are challenging to obtain, in part because the reporting mandates and follow-up periods for clinical trials have been limited. ${ }^{224}$ One retrospective analysis of data safety reporting from 194 published immunotherapy clinical trials across disease settings spanning 10 years identified 23 qualifying cases 
of irAEs arising more than 90 days after the reporting window. ${ }^{225}$ Data are even sparser on delayed onset-irAEs in patients with breast cancer receiving immunotherapies due to the relatively recent introduction of these treatments into clinical use. It is important to emphasize that immune effects can occur within a week to more than 1 year after initiation of therapy (including after cessation of therapy, and even after exposure to a single dose), so monitoring over a period of 12-24 months for symptoms of immune toxicities following therapy initiation is recommended. Further study on this topic is needed.

\section{Rechallenging with ICI after irAE: when is retreatment appropriate?}

The decision to rechallenge patients with immunotherapy following an irAE depends on the perceived benefit versus risk for the patient. The risk-benefit calculation for a patient with a symptomatic irAE should be based on the severity of the event, time to recovery to grade 1 or lower toxicity, the ability to taper off steroids without recurrence of toxicity, and if immunotherapy is clearly providing clinical benefit. Most expert guidelines, including from SITC, ${ }^{220}$ recommend permanent cessation of immunotherapy agents for most grade 3-4 toxicities and potential rechallenge for grade 2 AEs that resolve to grade $0-1$ promptly with supportive therapy. ${ }^{215} 217226$ Some exceptions to this rule are continuation of therapy for stable endocrinopathies and rechallenge in cases of grade 3 colitis, as only roughly $30 \%$ of patients develop recurrent colitis after retreatment with anti-PD-1 ICI. ${ }^{227}$ Conversely, treatment is permanently stopped for grade 2 cardiac and neurologic complications. Currently, there are no data to suggest that patients with prior PD-(L) 1 treatment are less likely to respond to rechallenge. Although the onset of irAEs on therapy has been linked with improved OS and PFS for some tumor types, ${ }^{228}$ special consideration is warranted about the value of continuing therapy for patients with breast cancer as the overall clinical benefits with ICI (particularly for PFS) are more modest when compared with other immune-responsive tumor types.

\section{Other general concerns for patients with breast cancer treated with immunotherapy}

Curative locoregional therapy including definitive surgery and adjuvant radiation therapy have been used sequentially or concurrently with anti-PD-(L) 1 immunotherapy in the I-SPY2, ${ }^{65}$ KEYNOTE-522, ${ }^{70}$ and IMpassion $031^{77}$ studies. Of note, for immunotherapy in the adjuvant setting, monitoring for irAEs may be challenging if patients transfer to another healthcare provider. For example, if a patient receives adjuvant therapy at a tertiary care center, but then transfers care to a local oncologist, it is essential there is exchange of information among providers regarding the risk of toxicity and need for ongoing vigilance for irAEs. In published trials, there has not been an increased incidence of perioperative complications with immunotherapy. ${ }^{6568229}$ While rare with PD-(L) 1 agents, adrenal insufficiency is associated with ICIs, and therefore obtaining a preoperative cortisol level is recommended for patients who have received neoadjuvant checkpoint inhibitors.

Pneumonitis is associated with receipt of prior thoracic or chest wall/breast radiation, and checkpoint inhibitor therapy may increase risk for pulmonary toxicities. ${ }^{230} 231$ Patients with breast cancer undergoing post-mastectomy or regional nodal irradiation are exposed to some level of radiation dose to the lungs, but the rates of pneumonitis did not appear significantly higher in the patients receiving adjuvant pembrolizumab concurrent with adjuvant radiation in KEYNOTE-522. ${ }^{70}$ Additional data are required to better understand how different radiation techniques (eg, protons, intensity modulated radiation therapy, volumetric arc therapy, and dose and fractionation regimen) may affect the risk of pneumonitis over time when radiation is delivered concurrently or in close proximity with ICIs. No data exist regarding the specific impact of adjuvant immunotherapy on breast reconstruction outcomes or cosmesis, including in patients who received radiation. Radiation techniques may be relevant in terms of potential impact on the risk of developing lymphopenia, particularly when radiation therapy is given with immunotherapy to large target volumes.

Finally, patients with cancer are at increased risk for severe complications with influenza infection. ${ }^{232} 233$ Several retrospective analyses have demonstrated that inactivated influenza vaccines are safe and effective in patients being treated with ICIs. ${ }^{234}{ }^{235}$ Vaccination with clinically indicated vaccines (eg, seasonal influenza, COVID-19) should be encouraged. Currently, data are lacking on the safety of live-attenuated vaccines in the context of checkpoint blockade specifically, but current recommendations for patients with cancer undergoing immunosuppressive therapy state that live-attenuated vaccines should be administered $\geq 4$ weeks prior to onset or $\geq 3$ months after immune restoration. ${ }^{236}$

\section{Panel recommendations}

- In patients with pre-existing comorbidities, active autoimmune disease requiring systemic immunosuppression (>10 mg prednisone equivalent or biologics), or those who have experienced toxicities with prior therapies, the benefits of immunotherapy must be weighed against the potential for severe AEs.

- Patients should be monitored for symptoms of immune toxicities during immunotherapy and for at least 12 months after discontinuation of treatment. Importantly, irAEs may occur after immunotherapy has been discontinued and other therapy initiated (LE: 1).

- For patients with early stage TNBC who receive pembrolizumab, serum cortisol should be tested at baseline, prior to surgery, and as clinically indicated.

- For patients with breast cancer who experience irAEs during immunotherapy treatment, management should generally follow the most updated guidelines 
(eg, SITC, ASCO, National Comprehensive Cancer Network (NCCN)) as this field is rapidly evolving.

- For patients with breast cancer who develop thyroid disorders or adrenal insufficiency while on treatment, immunotherapy can generally be continued (LE: 2).

\section{PATIENT EDUCATION AND QOL Patient and caregiver education}

ICIs represent a new treatment option for metastatic TNBC. As more patients with breast cancer receive ICI therapy, it is crucial for all members of a care team to be knowledgeable about the unique toxicity profile associated with immunotherapy compared with conventional oncology agents and to take into account a holistic view of QOL during and after treatment.

The potential for toxicity underscores the importance of open communication among the patient, family, and treating oncology team. ${ }^{237}$ Patient education is critical, including how immunotherapy eliminates cancer, how it is administered, and the potential for irAEs. ${ }^{215} 218$ Patients need to understand that, due to the different way immunotherapy eliminates cancer, many toxicities that the patient may experience are different than what is experienced with chemotherapy or hormonal therapy (for detailed descriptions of irAEs, see the Patient selection and toxicity management section). This is particularly important when talking to patients with metastatic breast cancer who have experience with chemotherapy or endocrine therapy and may expect dose reduction in the event of toxicity, as opposed to withholding ICI. It may be useful to use metaphors to communicate with patients, such as describing how ICIs essentially take off the 'brakes' of the immune system and thereby enable an antitumor immune response. Patients may have to make difficult decisions about their treatment. Caregivers also provide support to patients during cancer treatment, so it is important to include caregivers and other family members when providing education to patients about immunotherapy.

Currently, PD-(L) 1 inhibition is only FDA-approved for metastatic TNBC; however, there are many clinical trials testing immunotherapies in early-stage breast cancer. ${ }^{65} 74$ Patients with metastatic breast cancer report interest in ongoing side effects and how therapy will interfere with daily living long-term. ${ }^{238}{ }^{239}$ In this setting, it is reassuring that patient-reported outcomes (PRO) demonstrated no impact on health-related quality of life (HRQOL) and day-to-day functioning when atezolizumab was added to nab-paclitaxel compared with the group receiving chemotherapy only in IMpassion $130 .{ }^{240}$ In patients with earlystage breast cancer, the decision to pursue adjuvant and/ or neoadjuvant therapy may be difficult and must include a discussion about the possibility of acute irAEs and the potential for long-term (and perhaps unknown) immunerelated toxicities after treatment has ended. In particular, referral of patients with endocrinopathies (particularly adrenal insufficiency) to endocrinology for follow-up is recommended. Patients with adrenal insufficiency must be educated on the importance of regular-dosing and stress-dosing of steroids to avoid life-threatening adrenal crises, ${ }^{241}$ and may want to consider obtaining a MedicAlert bracelet. Medical records/summary of care documents should be updated to reflect the diagnosis.

For patients with early-stage breast cancer in particular, it is important to discuss impact on fertility. Although robust data on whether immunotherapy directly has adverse effects on conception and gestation are currently lacking $^{242}$ (with the exception of two isolated case reports of pregnancies successfully carried to term during treatment with ICIs for melanoma ${ }^{243} 244$ ), immune-related endocrinopathies may have long-term consequences on fertility. If available, an oncofertility consultation prior to initiation of immunotherapy may assist patients in making decisions regarding their future ability to have children. ${ }^{24}$ Also, immunotherapy may affect pituitary function, which can result in an inability to lactate or galactorrhea. Autoimmune hypophysitis in healthy women is rare, but occurs more frequently in women who are or were pregnant. ${ }^{246}$ It is not known if prior immunotherapy affects this risk in women of childbearing age who go on to conceive after therapy is completed.

NCCN guidelines recommend that patients use effective birth control during and for at least 5 months following completion of immunotherapy treatments, and many clinical trials mandate the use of contraception for up to 6 months after the final dose on study. However, the data supporting these recommendations are limited. ${ }^{242}$ Checkpoint inhibitor therapy is considered category $\mathrm{D}$ in pregnancy. ${ }^{247}$ Additionally, if a woman requires treatment with steroid-sparing immunosuppressive agents such as mycophenolate while on ICI therapy, risk of fetal malformation is increased. ${ }^{248} 249$ Despite the two isolated case reports of successful conception and viable pregnancies during ICI therapy mentioned above, ${ }^{243} 244$ due to limited safety data, initiation of checkpoint therapy during pregnancy is discouraged.

\section{QOL and symptom monitoring}

Currently, the majority of experience with immunotherapy for breast cancer has been in the advanced/ metastatic setting. Patients with metastatic breast cancer can experience an accumulation of physical symptoms and psychosocial stressors that adversely affect their QOL throughout their continued treatment. Over time, these effects usually become worse as treatment is ongoing. ${ }^{238}{ }^{239}$ A robust corpus of literature has described key QOL outcomes for patients receiving chemotherapy, radiotherapy, endocrine therapy, or HER2-directed therapies. In addition, throughout a patient's journey, multiple intrinsic and extrinsic factors may influence QOL, including AEs associated with therapy as well as other characteristics of the individual being treated (eg, menopausal status and socioeconomic status). ${ }^{250-253}$ However, data are currently lacking on the QOL implications for the addition of ICIs to chemotherapy or other conventional treatments. 
Ongoing trials are also evaluating ICIs in early-stage disease. Patients with early-stage breast cancer also experience both physical symptoms and psychosocial stressors that can adversely affect their QOL. ${ }^{254-256}$ Although survivors of early-stage breast cancer generally report high functioning after the conclusion of treatment, important rehabilitation problems may persist beyond 1 year after primary treatment, including difficulties with physical and recreational activities, body image, sexual interest, sexual function, and dating for those who were single. ${ }^{238}$

Although QOL data are limited in patients with breast cancer who received immunotherapy, the use of adjuvant immunotherapy in other cancers can help inform what to expect. Data from other malignancies suggests that, due to the primed immune system, toxicities may be greater in the adjuvant setting than in the advanced disease setting. ${ }^{257}$ However, PRO data from IMpassion031 showed no meaningful differences in HRQOL outcomes between the control and immunotherapy arms. ${ }^{258}$ Regardless, if the adjuvant treatment landscape for breast cancer expands to include ICIs (for more details on ongoing studies, see the Emerging data on immunotherapy with PD-(L) 1 inhibitors for early-stage/locally advanced breast cancer section), the decision to recommend must be carefully considered, and patients must be active participants in the decision-making process. Knowing that patients with early-stage disease are typically healthy prior to their diagnosis, early identification of irAEs is imperative to minimize the detrimental effects of QOL on treatment. Additionally, the potential for long-term toxicity affecting physical activity and daily living means that their overall QOL may be impacted by immunotherapy treatment. Therefore, assessing for the early or subtle signs and symptoms of irAEs is critical for prompt diagnosis and management.

No significant effects on HRQOL were observed with the addition of atezolizumab to nab-paclitaxel for the treatment of metastatic TNBC in IMpassion $130 .{ }^{259} \mathrm{~A}$ separate analysis of HRQOL in patients with metastatic TNBC randomly assigned to receive either pembrolizumab or chemotherapy in the KEYNOTE-119 trial found benefits for immunotherapy over standard of care in all PRO endpoints. Among the patients with PD-L1 expression in tumors and immune-infiltrating cells, the median time to QOL deterioration as measured by PRO was 4.3 months for pembrolizumab versus 1.7 months with chemotherapy (HR $0.70 ; 95 \%$ CI 0.46 to 1.05 ). Additionally, deterioration in fatigue, nausea and vomiting, pain, dyspnea, and loss of appetite were all observed with chemotherapy but remained stable or improved slightly with immunotherapy. ${ }^{260}$

It will be important to gather data on HRQOL in future trials, and to include assessments with validated tools to enable meaningful comparisons across studies. Currently, the European Organization for Research and Treatment of Cancer (EORTC) Quality of Life Questionnaire (EORTC QLQ-C30) and the European Quality of Life 5 Dimension (EQ-5D) are the most commonly used
HRQOL assessment instruments in immunotherapy trials. $^{261}$

\section{Panel recommendations}

- For patients receiving immunotherapy, education should be provided, including the differences between chemotherapy and immunotherapy. Whenever possible, caregivers and family members should be included in these conversations.

- Patients should be encouraged to use contraception while receiving immunotherapy, and a discussion about fertility should be initiated prior to treatment.

- Patients and providers should be educated about potential irAEs, including the expected timing of symptom onset and management of toxicity with immunotherapies, rationale for holding doses as opposed to dose reductions, and detailed parameters for when to contact their care team.

- For patients being treated with immunotherapy, education should include the importance of early recognition and management of irAEs, emphasizing that some of the more common toxicities have vague symptoms and therefore any change from baseline health should be reported. Additionally, patients should be encouraged to inform all their current and future healthcare providers that they have been treated with immunotherapy.

\section{NOVEL COMBINATION STRATEGIES AND INTRIGUING FUTURE DIRECTIONS}

A number of ongoing trials are evaluating novel immunotherapeutic strategies, including new targets, emerging agents like bispecific antibodies, and combinations of checkpoint inhibitors with other treatment modalities such as radiotherapy, cryotherapy, or targeted drugs. Although none of these emerging strategies have gained FDA approval at this time, if results from early studies are encouraging, they may develop into feasible options for patients with breast cancer in the near future.

\section{Radiotherapy}

Combining ICIs with local, ablative therapies is a strategy that has garnered substantial enthusiasm. This is in part due to the potential for radiation to elicit systemic immune responses, as well as advances in sophisticated radiation oncology technologies such as stereotactic radiation, which permits high, ablative doses of radiation to be tailored to the tumor volume while minimizing damage to surrounding normal tissue. ${ }^{190}$ Although the bulk of evidence so far is preclinical, ${ }^{262} \mathrm{a}$ few trials have evaluated the integration of radiotherapy with checkpoint inhibition in patients with breast cancer. In a phase II study evaluating the combination of hypofractionated radiotherapy and pembrolizumab in 17 patients with metastatic TNBC unselected for PD-L1 status and heavily pre-treated with prior lines of chemotherapy, the ORR for the entire cohort was $17.6 \%$ with $100 \%$ reduction in tumor volume outside of the irradiated portal among responders. ${ }^{263}$ 
Treating earlier in the disease course and PD-L1 status appeared to be predictors of response. Another phase II trial that enrolled eight patients with heavily pre-treated (median two prior lines of chemotherapy) metastatic HR+ breast cancer for treatment with radiotherapy plus pembrolizumab demonstrated no objective responses and halted accrual after the first cohort. ${ }^{191}$

The strategy of combining radiation with ICIs and/or other novel immune targets in the preoperative setting for TNBC and HR+/HER2- breast cancer is currently being evaluated in four clinical trials. ${ }^{264}$ The largest study, P-RAD: A Randomized Study of Preoperative Chemotherapy, Pembrolizumab and No, Low or High Dose RADiation in Node-Positive, HER2-Negative Breast Cancer (NCT04443348), is evaluating two co-primary endpoints of change in TILs and pathologic nodal response as a surrogate for the abscopal effect in patients with nodepositive, HER2- breast cancer who receive neoadjuvant pembrolizumab, chemotherapy and radiation to the intact breast tumor. Another endpoint of this trial is to compare different radiation modalities, protons and photons, in combination with pembrolizumab. Relative to standard photon therapy, proton therapy is a highly precise form of radiation that is known for sharp dose fall-off beyond the tumor target, which allows sparing of T-lymphocytes, hypothetically leaving them available to generate a robust immune response. Other questions regarding the optimal sequencing, timing, and modality of radiation to integrate with immunotherapy remain active topics of investigation.

\section{Cryotherapy}

Feasibility and safety of cryotherapy in combination with ICIs was shown in a pilot study of 19 women with breast cancer for whom mastectomy was planned where patients were treated with preoperative tumor cryoablation $(\mathrm{n}=7)$, single-dose ipilimumab at $10 \mathrm{mg} / \mathrm{kg}(\mathrm{n}=6)$, or both $(n=6)$. The regimens were all safe and tolerable, and combination therapy was associated with sustained peripheral elevations in Th1-type cytokines, activated and proliferating $\mathrm{CD}^{+}$and $\mathrm{CD} 8^{+} \mathrm{T}$ cells, and a favorable ratio of proliferative effector $\mathrm{T}$ cells relative to regulatory $\mathrm{T}$ cells within the tumors. ${ }^{265}$ A phase II study of ipilimumab, nivolumab, and cryoablation for patients with $\geq 1 \mathrm{~cm}$ of residual TNBC after standard of care chemotherapy is underway (NCT03546686).

\section{DNA repair-based therapies}

Combining immunotherapy with PARP inhibitors is also an appealing strategy that is currently being explored in multiple trials. In breast cancers, loss of function mutations in BRCA1 or BRCA2 lead to dependence on PARP for the repair of double-stranded DNA breaks, which has led to the successful clinical use of agents such as olaparib and talazoparib, selective, orally available PARP-inhibitors that are FDA-approved for germline BRCA1/2-mutated metastatic breast cancer. ${ }^{90} 110111$ The sustained DNA damage that accumulates as a result of PARP inhibition can also drive the emergence of neoantigens as well as the upregulation of interferons in the tumor microenvironment due to cGAS/STING sensing in repaircompetent tumors, possibly potentiating the effects of immunotherapy ${ }^{266}$ Based on that rationale, the phase II TOPACIO/KEYNOTE-162 trial evaluated the combination of the PARP inhibitor niraparib and pembrolizumab in 54 patients with metastatic TNBC, only 12 of whom (22\%) had a deleterious BRCA1/2 mutation. At the time of data analysis, 45 patients were evaluable and the ORR was $29 \%$ with a disease control rate (DCR) of $49 \%$, including 3 CR (7\%), 10 PR (22\%), 9 SD (20\%), and 23 cases of progressive disease $(51 \%) .{ }^{267}$ Best responses were observed in patients with a tumor BRCA1/2 mutation. In the phase II single arm MEDIOLA trial, patients with germline BRCA1/2 mutations achieved a DCR of $80 \%$ at 12 weeks using the combination of olaparib and durvalumab. ${ }^{268}$ In a recently reported arm in the I-SPY2 trial, the addition of durvalumab and olaparib to weekly paclitaxel treatments increased pCR rates across all biomarker subsets of breast cancer (HER2-, 22\% vs 37\%; ER+/HER2- Mammaprint High Risk, 14\% vs 28\%; TNBC, $27 \%$ vs $47 \%) .{ }^{73}$ Future trials will be important to define not only the depth of response, but the durability of these responses as well.

\section{Anti-VEGF and tyrosine kinase inhibitors}

Combination trials evaluating checkpoint inhibitors with anti-VEGF agents and tyrosine kinase inhibitors are also ongoing. The non-randomized, phase II NEWBEAT study reported results for the addition of nivolumab to the combination of paclitaxel and the anti-angiogenic monoclonal antibody, bevacizumab, for the first-line treatment in patients with metastatic HER2- breast cancer. The OS rate at 12 months was $87.1 \%$ and the ORR was $75.4 \%$ in patients with ER+ tumors and $83.3 \%$ in patients with TNBC. Median PFS was not yet reached at the time of reporting, but PFS rate at 12 months was $75.8 \%{ }^{269}$

\section{CDK 4/6 inhibitors}

Preclinical studies demonstrate that CDK 4/6 inhibition promotes antitumor immunity by increasing antigen processing and presentation. ${ }^{270}$ Initial results of an ongoing phase Ib study of pembrolizumab plus the CDK 4/ 6 inhibitor, abemaciclib, showed a tolerable safety profile and potential clinical benefit, with a $14.3 \%$ ORR and a $60 \%$ rate of SD at 16 weeks. ${ }^{271}$ In a follow-up analysis that included 26 patients, the DCR was $77 \%$ and clinical benefit rate $(\mathrm{CR}+\mathrm{PR}+\mathrm{SD}$ persisting for $\geq 6$ months) was $27 \%$. Although grade 3 and grade $4 \mathrm{AEs}$ were generally reversible following drug holds and corticosteroids, preliminary results in a phase Ib study reported two fatal events as a result of pneumonitis and 15 patients $(58 \%)$ who discontinued treatment. ${ }^{272}$ Another study, the phase II NEWFLAME trial, which evaluated nivolumab in combination with abemaciclib plus endocrine therapy in patients with HR+/HER2- metastatic breast cancer ${ }^{273}$ was discontinued early due to safety concerns. ${ }^{274}$ The phase II CheckMate 7A8 study that is investigating nivolumab plus 
the CDK 4/6 inhibitor palbociclib plus anastrozole in postmenopausal women and men with ER+/HER2- primary breast cancer ${ }^{275}$ is ongoing. As these studies are ongoing, caution should be made when considering the tolerability of these combinations, which may differ based on the specific CDK 4/6 inhibitors and/or ICIs being considered.

\section{Bispecific T cell engagers}

One factor potentially underlying breast cancer immune evasion is the downregulation or total loss of human leukocyte antigen (HLA) class I on TCs. ${ }^{276-278}$ One strategy to overcome loss of antigenicity by TCs involves bispecific antibodies that bridge T cell CD3 and cancer cell surface markers. Several bispecifics directed against breast cancerspecific antigens are currently in development, with some reporting tolerable safety and preliminary efficacy in human trials. For instance, in a phase I study of PRS-343, the first HER2/4-1BB bispecific molecule to enter human trials, a DCR of $58 \%$ (with $11 \%$ confirmed PR per RECIST v1.1) was reported among the 19 of 51 patients with a variety of solid tumors (including 12 with breast cancer) who were evaluable at the time of analysis. No serious AEs were reported. ${ }^{279}$

\section{Adenosine receptor inhibitors}

Adenosine is an immunosuppressive metabolite produced at high levels within the tumor microenvironment. Hypoxia, high cell turnover, and expression of CD39 and CD73 are important factors in adenosine production. ${ }^{280}$ Metabolic reprogramming has been linked to the emergence of treatment resistance in breast cancer. ${ }^{281} 282$ As one example, activation of $\mathrm{A} 2 \mathrm{aR}$ or another adenosine receptor, A2bR, suppresses $\mathrm{T}$ cell proliferation, cytokine production, and cytotoxicity, and new agents such as the adenosine receptor inhibitor, CPI-444, are being evaluated in combination with checkpoint inhibition. ${ }^{283}$

\section{Vaccines}

Therapeutic vaccines or intratumoral therapies for breast cancer have been evaluated in early-phase and randomized trials. One feasibility study of cyclophosphamide, trastuzumab, and an allogeneic granulocyte-macrophage colonystimulating factor (GM-CSF)-secreting breast tumor vaccine for HER2+ metastatic breast cancer enrolled 20 patients and reported median PFS and OS of 7 months (95\% CI 4 to 16) and 42 months (95\% CI 22 to 70), respectively. ${ }^{284}$ Another phase I/II study of concurrent HER2-specific vaccination in 22 patients with stage IV HER2+ tumors demonstrated epitope spreading to additional tumor-related proteins after immunization. ${ }^{285}$ Enthusiasm for the vaccine approach diminished somewhat after the phase III multicenter clinical trial of the sialyl-TN (STn)-keyhole limpet hemocyanin (KLH) vaccine for metastatic breast cancer demonstrated no significant benefit in time to progression in 1,028 women. ${ }^{286}$ Currently, several ongoing studies are evaluating intratumoral oncolytic viral therapy, including, but not limited to, pelareorep talimogene laherparepvec (T-VEC), and PVX410 with pembrolizumab in HLA-A2+ metastatic TNBC (NCT03362060).

\section{Other immune targets}

Targeting additional mechanisms of tumor immune evasion is critical to extending the benefits of immunotherapy to breast cancer. Although the majority of published studies on immunotherapy for breast cancer have evaluated agents targeting the PD-(L) 1 axis, some trials have reported initial efficacy with other immunotherapy targets. A phase I study that evaluated anti-CTLA-4 tremelimumab plus exemestane in 26 patients with advanced, hormone-responsive breast cancer found favorable safety, with most treatment-related AEs being mild-to-moderate, and a best overall response of $\mathrm{SD} \geq 12$ weeks in 11 patients $(42 \%) .{ }^{287}$ Other strategies that remain under investigation in early-phase trials include combining checkpoint blockade with other agents modulating various targets, including the lymphocyte-activation gene 3 (LAG-3), TIGIT, and the T cell agonist OX40.

\section{Panel recommendations}

- Given the limited activity with currently available single-agent immunotherapy, the efficacy of immunotherapeutic strategies will likely be enhanced with combination therapy adding chemotherapy, targeted therapies, radiotherapy, or other immunotherapy agents.

- Based on current evidence, the combinations mentioned above are investigational and should only be considered in the context of a clinical trial.

- The optimal dose of radiation (low or high) to combine with ICIs in the preoperative setting is the subject of an ongoing clinical trial (NCT04443348). Data from this trial will permit design of large, phase II trials examining radiation and immunotherapy combinations in the pre-operative setting.

- In ongoing and planned studies involving combination approaches with immunotherapy, both shortterm and long-term toxicities should be a careful consideration.

- Companion biomarkers that predict clinical benefit and/or toxicity are essential in the development of these strategies.

\section{CONCLUSION}

Immunotherapy is now offering extended survival to patients with TNBC, a subset of breast cancer patients who formerly had very few treatment options. Despite these advances, many patients with breast cancer are ineligible for immunotherapy in the standard of care setting. As additional trials continue to report results, the outlook may further improve for patients with earlier stages of TNBC or other disease subtypes. Future trials are needed to address the impact of immunotherapy in HR+ and HER2+ subtypes as well as the optimal chemotherapy partner(s) for ICIs, especially as oncologists and patients balance the potential for harm and benefit in early-stage cancer. Furthermore, more studies will be needed to determine the best options for patients who relapse after initial treatment with immunotherapy. Careful consideration should also be given to tissue choice and assay choice for biomarker assessment, and additional 
study is needed to determine the optimal biomarker(s) for ICIs in breast cancer. In the future, the indications for existing immunotherapies are likely to continue to expand, and novel combinations may be approved. It is an exciting and dynamic time for immunotherapy in breast cancer, and these guidelines will be updated as the field continues to evolve.

\section{Author affiliations}

${ }^{1}$ Department of Medicine, UPMC Hillman Cancer Center, University of Pittsburgh,

Pittsburgh, Pennsylvania, USA

${ }^{2}$ Perlmutter Cancer Center, New York University Langone, New York, New York, USA

${ }^{3}$ Department of Pathology and Oncology, Johns Hopkins University School of

Medicine, Baltimore, Maryland, USA

${ }^{4}$ Cancer Vaccine Institute, University of Washington, Seattle, Washington, USA

${ }^{5}$ Pelotonia Institute for Immuno-Oncology, Division of Medical Oncology, The Ohio

State University Comprehensive Cancer Center, Columbus, Ohio, USA

${ }^{6}$ Department of Radiation Oncology, Massachusetts General Hospital, Boston,

Massachusetts, USA

${ }^{7}$ Winship Cancer Institute, Emory University, Atlanta, Georgia, USA

${ }^{8}$ Department of Medicine, UT Southwestern, Dallas, Texas, USA

${ }^{9}$ Division of Breast Surgery, Department of Surgery, Brigham and Women's Hospital, Boston, Massachusetts, USA

${ }^{10}$ Breast Oncology Program, Dana-Farber Cancer Institute, Harvard Medical School, Boston, Massachusetts, USA

${ }^{11}$ Department of Medicine, Section of Hematology/Oncology, The University of Chicago Medicine Comprehensive Cancer Center, Chicago, Illinois, USA

${ }^{12}$ Earle A Chiles Research Institute, Portland, Oregon, USA

${ }^{13}$ Helen Diller Family Comprehensive Cancer Center, University of California San

Francisco, San Francisco, California, USA

${ }^{14}$ Center for Melanoma, Massachusetts General Hospital Cancer Center, Boston, Massachusetts, USA

${ }^{15}$ Department of Breast Oncology, H Lee Moffitt Cancer Center and Research Institute, Tampa, Florida, USA

${ }^{16}$ University of North Carolina Lineberger Comprehensive Cancer Center, Chapel Hill, North Carolina, USA

${ }^{17}$ Department of Medical Oncology, Dana-Farber Cancer Institute, Boston,

Massachusetts, USA

${ }^{18}$ Department of Breast Medical Oncology, Division of Cancer Medicine, The University of Texas MD Anderson Cancer Center, Houston, Texas, USA

Correction notice This article has been corrected since it was first published. On August 27, 2021, the indication for atezolizumab in combination with nab-paclitaxe as treatment for patients with triple-negative breast cancer whose tumors express PD-L1 was voluntarily withdrawn by the manufacturer. Amendments have been made to this article in light of the withdrawal.

Twitter Leisha A Emens @EmensLeisha, Margaret E Gatti-Mays @DrGattiMays, Heather L McArthur @hmcarthur, Rita Nanda @RitaNandaMD and Jennifer K Litton @jenniferlitton

Acknowledgements The authors acknowledge SITC staff for their contributions to the development and publication of this guideline including Sam Million-Weaver, $\mathrm{PhD}$, for medical writing support; Lionel Lim for project management assistance; and Angela Kilbert and Emily Gronseth, PhD, for editorial support. Additionally, the authors wish to thank the society for supporting the manuscript development.

Contributors All authors served on the SITC Breast Cancer Immunotherapy Guideline Expert Panel, drafted content, and provided critical review during the manuscript development. LAE and JKL provided leadership as Chairs of the Expert Panel and provided guidance on the manuscript structure and content and thus are first and last authors; all other authors are listed alphabetically by last name. PAS was the patient advocate representative.

Funding The authors have not declared a specific grant for this research from any funding agency in the public, commercial or not-for-profit sectors.

Competing interests SA-Contracted research: Funding to institution from Amgen, Bristol Myers Squibb, Merck, Celgene, Roche. AC-M-Consulting fees: Bristol Myers Squibb, Roche Diagnostics; Contracted research: Bristol Myers Squibb; Partner Salary: Vivante Health; Royalty: Springer/Demos Publishing-Textbooks. MLD-
Contracted research: Pfizer, EMD Serono, Bavarian Nordisk, Precigen, Epithany, Silverback Therapeutics, Celgene; IP Rights: University of Washington; Non-CME Services: SITC; Ownership interest: Epithany; Partner ownership interest: Epithany; Partner salary: Cox Cable; Royalty: University of Washington; Salary: University of Washington. LAE-Contracted research: Aduro Biotech, AstraZeneca, Bristol Myers Squibb, Corvus, EMD Serono, Genentech, F Hoffman La Roche, Maxcyte, Merck, Tempest, Silverback, Bolt, Compugen, Takeda, CytomX; Consulting fees: Genentech, F Hoffman La Roche, Syndax, Lilly, AbbVie, Amgen, AstraZeneca, Bayer, Bristol Myers Squibb, Celgene, Chugai, F Hoffman La Roche, GCPR, Genentech, Gilead, Gritstone, Medimmune, Macrogenics, Novartis, Peregrine, Replimune, Shionogi, Silverback, Vaccinex; IP Rights: Aduro Biotech; Royalty: Elsevier; Salary: University of Pittsburgh, UPMC UPP; Grants from non-industry entities: HeritX Incorporated, NSABP Foundation, Translational Breast Cancer Research Consortium, Breast Cancer Research Foundation, National Cancer Institute, Department of Defense, Johns Hopkins University, University of California San Francisco, Cornell University; Ownership interest: Molecuvax-potential for royalties in the future. MG-M-Trial funding to institution: EMD Serono (OSU Site PI). AYH-Consulting fees: Seattle Genetics; La Roche-Posay; Contracted research: Merck and GSK. KK-Consulting fees: Eli Lilly, Pfizer, Novartis, Eisai, AstraZeneca, Merck, Seattle Genetics; Contracted research: Incyte, Genentech, Eli Lilly, Pfizer, Calithera Biosciences, Acetylon, Seattle Genetics, Amgen, ZenoPharmaceuticals, CytomX Therapeutics; Partner Salary: Pfizer, Array Biopharma - no longer employee at either; Speaker Bureau: Eli Lilly. JKL-Contracted research: Novartis, Medivation/Pfizer, Genentech, GSK, EMD Serono, AstraZeneca, Medimmune, Zenith, Jounce (All were payments to my Institution of for writing support for manuscripts of multicenter trials. No payments directly to Dr Litton); Consulting fees: Pfizer/Medivation, AstraZeneca, Ayala (All honorariums were refused); Salary: The University of Texas MD Anderson Cancer Center. HLM-Consulting fees: Bristol Myers Squibb, Eli Lilly, Genentech/ Roche, Merck, Pfizer, Puma, Daiichi Sankyo, Seattle Genetics, AstraZeneca; Contracted Research: Bristol Myers Squibb, Medlmmune, LLC/AstraZeneca, BTG, Merck. EAM-Consulting fees: Merck, Genomic Health, Roche/Genentech; Contracted research: GlaxoSmithKline; NPI: 1831388596. RN-Consulting fees: Clovis, Immunomedics, Macrogenics, Merck, Pfizer, Seattle Genetics; Contracted research: AstraZeneca, Celgene, Corcept Therapeutics, Genentech/Roche, Immunomedics, Merck, OBI Pharma, Odonate Therapeutics, Pfizer, Seattle Genetics; DSMB: G1 Therapeutics. DBP-Consulting fees: Genentech, Merck, Brooklyn Immunotherapeutics; Contracted research: Merck, Brooklyn Immunotherapeutics, Bristol Myers Squibb; Speaker bureau: Genentech, Novartis. HSR-Consulting fees: Puma, Samsung - Limited consulting; Contracted research: Pfizer, Merck, Novartis, Lilly, Genentech, OBI, Odonate, Daiichi, Seattle Genetics, Eisai, Macrogenics, Immunomedics; Travel support for educational programs: Daiichi, Mylan, Pfizer, Merck, AstraZeneca, Novartis, Macrogenics. KMR-Consulting fees: Merck, Bristol Myers Squibb, Eisai. HS-Consulting fees: AstraZeneca, Eisai, Novartis, Celgene, PUMA, Seattle Genetics. PAS-Consulting fees: Pfizer. SMT-Consulting fees: AstraZeneca, Eli Lilly, Merck, Nektar, Novartis, Pfizer, Genentech, Immunomedics, Bristol Myers Squibb, Eisai, Nanostring, Puma, Sanofi, Celldex, Paxman, Odonate, Seattle Genetics, Silverback Therapeutics, G1 Therapeutics, AbbVie, Anthenex, Oncopep; Contracted research: AstraZeneca, Eli Lilly, Merck, Nektar, Novartis, Pfizer, Genentech, Immunomedics, Bristol Myers Squibb, Eisai, Nanostring, Sanofi, Exelisis, Seattle Genetics, Cyclacel, Odonate. SITC Staff: SMW—Shares owned: Pacific Biosciences of California, Editas Medicine. EG, AK, LL—Nothing to disclose.

Patient consent for publication Not required.

Provenance and peer review Not commissioned; externally peer reviewed.

Open access This is an open access article distributed in accordance with the Creative Commons Attribution Non Commercial (CC BY-NC 4.0) license, which permits others to distribute, remix, adapt, build upon this work non-commercially, and license their derivative works on different terms, provided the original work is properly cited, appropriate credit is given, any changes made indicated, and the use is non-commercial. See http://creativecommons.org/licenses/by-nc/4.0/.

\section{ORCID iDs}

Leisha A Emens http://orcid.org/0000-0002-7694-0731

Ashley Cimino-Mathews http://orcid.org/0000-0002-0638-7969

Margaret E Gatti-Mays http://orcid.org/0000-0001-8914-2897

David B Page http://orcid.org/0000-0001-9264-4628

Hope S Rugo http://orcid.org/0000-0001-6710-4814

Sara M Tolaney http://orcid.org/0000-0002-5940-8671 


\section{REFERENCES}

1 Berry DA, Cronin KA, Plevritis SK, et al. Effect of screening and adjuvant therapy on mortality from breast cancer. N Engl J Med Overseas Ed 2005;353:1784-92.

2 Bleyer A, Welch HG. Effect of three decades of screening mammography on breast-cancer incidence. $N$ Engl J Med Overseas Ed 2012;367:1998-2005.

3 Helvie MA, Bevers TB. Screening mammography for average-risk women: the controversy and NCCN's position. J Natl Compr Canc Netw 2018;16:1398-404.

4 Munoz D, Near AM, van Ravesteyn NT, et al. Effects of screening and systemic adjuvant therapy on ER-specific us breast cancer mortality. J Natl Cancer Inst 2014;106. doi:10.1093/jnci/dju289. [Epub ahead of print: 2409 2014].

5 Zielonke N, Gini A, Jansen EEL, et al. Evidence for reducing cancerspecific mortality due to screening for breast cancer in Europe: a systematic review. Eur J Cancer 2020;127:191-206.

6 Vagia E, Mahalingam D, Cristofanilli M. The landscape of targeted therapies in TNBC. Cancers 2020;12. doi:10.3390/ cancers12040916. [Epub ahead of print: 0804 2020]

7 Emens LA. Breast cancer immunotherapy: facts and hopes. Clin Cancer Res 2018;24:511-20.

8 Oner G, Altintas S, Canturk Z, et al. Triple-Negative breast cancerRole of immunology: a systemic review. Breast J 2020;26:995-9.

9 Gatti-Mays ME, Balko JM, Gameiro SR, et al. If we build it they will come: targeting the immune response to breast cancer. NPJ Breast Cancer 2019;5:37.

10 Burugu S, Asleh-Aburaya K, Nielsen TO. Immune infiltrates in the breast cancer microenvironment: detection, characterization and clinical implication. Breast Cancer 2017;24:3-15.

11 Cimino-Mathews A, Foote JB, Emens LA. Immune targeting in breast cancer. Oncology 2015;29:375-85.

12 da Silva JL, Cardoso Nunes NC, Izetti P, et al. Triple negative breast cancer: a thorough review of biomarkers. Crit Rev Oncol Hematol 2020;145:102855.

13 Cimino-Mathews A, Thompson E, Taube JM, et al. Pd-L1 (B7-H1) expression and the immune tumor microenvironment in primary and metastatic breast carcinomas. Hum Pathol 2016;47:52-63.

14 Huang W, Ran R, Shao B, et al. Prognostic and clinicopathological value of PD-L1 expression in primary breast cancer: a metaanalysis. Breast Cancer Res Treat 2019;178:17-33.

15 Mittal D, Gubin MM, Schreiber RD, et al. New insights into cancer immunoediting and its three component phases--elimination, equilibrium and escape. Curr Opin Immunol 2014;27:16-25.

16 Chen DS, Mellman I. Oncology meets immunology: the cancerimmunity cycle. Immunity 2013;39:1-10.

17 Narayan P, Wahby S, Gao JJ, et al. FDA approval summary: Atezolizumab plus paclitaxel protein-bound for the treatment of patients with advanced or metastatic TNBC whose tumors express PD-L1. Clin Cancer Res 2020;26:2284-9.

18 Schmid P, Adams S, Rugo HS, et al. Atezolizumab and nabpaclitaxel in advanced triple-negative breast cancer. $N$ Engl J Med 2018;379:2108-21.

19 Merck. Pembrolizumab highlights of prescribng information. US FDA: Drugs@FDA, 2018.

20 Institute of Medicine Committee on Standards for Developing Trustworthy Clinical Practice Guidelines. Clinical practice guidelines we can trust. Washington, DC: National Academies Press (US), 2011.

21 Roche. Atezolizumab package insert. Silver Spring, Maryland: DRUGS@FDA, 2016.

22 Adams S, Diamond JR, Hamilton EP, et al. Phase Ib trial of atezolizumab in combination with nab-paclitaxel in patients with metastatic triple-negative breast cancer (mTNBC). Journal of Clinical Oncology 2016;34:1009.

23 Emens L, Loi S, Rugo HS, et al. Abstract GS1-04: IMpassion130: Efficacy in immune biomarker subgroups from the global, randomized, double-blind, placebo-controlled, phase III study of atezolizumab + nab-paclitaxel in patients with treatment-naïve, locally advanced or metastatic triple-negative breast cancer. Cancer Research 2019;79:GS1-04-GS1-04.

24 Schmid P, Rugo HS, Adams S, et al. Atezolizumab plus nabpaclitaxel as first-line treatment for unresectable, locally advanced or metastatic triple-negative breast cancer (IMpassion130): updated efficacy results from a randomised, double-blind, placebocontrolled, phase 3 trial. Lancet Oncol 2020;21:44-59.

25 Emens LA, Adams S, Barrios CH, et al. LBA16 IMpassion130: final OS analysis from the pivotal phase III study of atezolizumab + nabpaclitaxel vs placebo + nab-paclitaxel in previously untreated locally advanced or metastatic triple-negative breast cancer. Annals of Oncology 2020;31:S1148.
26 Cortes J, Cescon DW, Rugo HS, et al. KEYNOTE-355: randomized, double-blind, phase III study of pembrolizumab + chemotherapy versus placebo + chemotherapy for previously untreated locally recurrent inoperable or metastatic triple-negative breast cancer. Journal of Clinical Oncology 2020;38:1000.

27 Rugo HS, Cescon DW, Nowecki Z. Additional efficacy endpoints

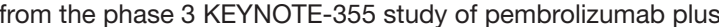
chemotherapy vs placebo plus chemotherapy as first-linetherapy for locally recurrent inoperable or metastatic triple-negative breast cancer. in 2020 San Antonio Breast Cancer Symposium 2020.

28 McGranahan N, Furness AJS, Rosenthal R, et al. Clonal neoantigens elicit $T$ cell immunoreactivity and sensitivity to immune checkpoint blockade. Science 2016;351:1463-9.

29 Yi M, Qin S, Zhao W, et al. The role of neoantigen in immune checkpoint blockade therapy. Exp Hematol Oncol 2018;7:28.

30 Heemskerk B, Kvistborg P, Schumacher TNM. The cancer antigenome. Embo J 2013;32:194-203.

31 Rooney MS, Shukla SA, Wu CJ, et al. Molecular and genetic properties of tumors associated with local immune cytolytic activity. Cell 2015;160:48-61.

32 Turajlic S, Litchfield K, Xu H, et al. Insertion-and-deletion-derived tumour-specific neoantigens and the immunogenic phenotype: a pan-cancer analysis. Lancet Oncol 2017;18:1009-21.

33 Hause RJ, Pritchard CC, Shendure J, et al. Classification and characterization of microsatellite instability across 18 cancer types. Nat Med 2016;22:1342-50.

34 Marcus L, Lemery SJ, Keegan P, et al. Fda approval summary: pembrolizumab for the treatment of microsatellite instability-high solid tumors. Clin Cancer Res 2019;25:3753-8.

35 Le DT, Uram JN, Wang H, et al. Pd-1 blockade in tumors with mismatch-repair deficiency. N Engl J Med 2015;372:2509-20.

36 Le DT, Kim TW, Van Cutsem E, et al. Phase II open-label study of pembrolizumab in treatment-refractory, microsatellite InstabilityHigh/Mismatch repair-deficient metastatic colorectal cancer: KEYNOTE-164. J Clin Oncol 2020;38:11-19.

37 Nanda R, Chow LQM, Dees EC, et al. Pembrolizumab in patients with advanced triple-negative breast cancer: phase $\mathrm{lb}$ KEYNOTE-012 study. J Clin Oncol 2016;34:2460-7.

38 Rugo HS, Delord J-P, Im S-A, et al. Safety and antitumor activity of pembrolizumab in patients with estrogen Receptor-Positive/Human epidermal growth factor receptor 2-Negative advanced breast cancer. Clin Cancer Res 2018;24:2804-11.

39 Marabelle A, Le DT, Ascierto PA, et al. Efficacy of pembrolizumab in patients with Noncolorectal high microsatellite Instability/Mismatch repair-deficient cancer: results from the phase II KEYNOTE-158 study. J Clin Oncol 2020;38:1-10.

40 FDA. FDA approves pembrolizumab for adults and children with TMB-H solid tumors. US FDA: Drug Approvals and Databases, 2020.

41 Kurata K, Kubo M, Mori H, et al. Abstract P1-06-11: microsatellite instability in triple negative breast cancers. Cancer Research 2019;79:P1-06-11-P1-06-11.

42 Cortes-Ciriano I, Lee S, Park W-Y, et al. A molecular portrait of microsatellite instability across multiple cancers. Nat Commun 2017;8:15180.

43 Ozer E, Yuksel E, Kizildag S, et al. Microsatellite instability in earlyonset breast cancer. Pathol Res Pract 2002;198:525-30.

44 Siah SP, Quinn DM, Bennett GD, et al. Microsatellite instability markers in breast cancer: a review and study showing MSI was not detected at 'BAT 25' and 'BAT 26' microsatellite markers in earlyonset breast cancer. Breast Cancer Res Treat 2000;60:135-42.

45 Anbazhagan R, Fujii H, Gabrielson E. Microsatellite instability is uncommon in breast cancer. Clin Cancer Res 1999;5:839-44.

46 Barroso-Sousa R, Jain E, Cohen O, et al. Prevalence and mutational determinants of high tumor mutation burden in breast cancer. Ann Oncol 2020;31:387-94.

47 Emens LA, Molinero L, Adams S, et al. 296P tumour mutational burden and clinical outcomes with first-line atezolizumab and nab-paclitaxel in triple-negative breast cancer: exploratory analysis of the phase III IMpassion130 trial. Annals of Oncology 2020;31:S360-1.

48 Alva AS, Mangat PK, Garrett-Mayer E, et al. Pembrolizumab (P) in patients (PTS) with metastatic breast cancer (MBC) with high tumor mutational burden (HTMB): results from the targeted agent and profiling utilization registry (TAPUR) study. Journal of Clinical Oncology 2019;37:1014

49 Winer EP, Lipatov O, Im S-A, et al. Association of tumor mutational burden (TMB) and clinical outcomes with pembrolizumab (pembro) versus chemotherapy (chemo) in patients with metastatic triplenegative breast cancer (mTNBC) from KEYNOTE-119. Journal of Clinical Oncology 2020;38:1013. 
50 Miles D, André F, Gligorov J, et al. Abstract OT1-01-01: IMpassion131: a phase III study comparing $1 \mathrm{~L}$ atezolizumab with paclitaxel vs placebo with paclitaxel in treatment-naive patients with inoperable locally advanced or metastatic triple negative breast cancer (TNBC). Cancer Research 2018;74:OT1-01-01-OT101-01.

51 Miles DW, Gligorov J, André F, et al. LBA15 primary results from IMpassion131, a double-blind placebo-controlled randomised phase III trial of first-line paclitaxel (PAC) \pm atezolizumab (atezo) for unresectable locally advanced/metastatic triple-negative breast cancer (mTNBC). Annals of Oncology 2020;31:S1147-8.

52 FDA. FDA issues alert about efficacy and potential safety concerns with atezolizumab in combination with paclitaxel for treatment of breast cancer, D. FDA, editor. Silver Spring, Maryland: US FDA, 2020.

53 Cortés J, André F, Gonçalves A, et al. IMpassion132 phase III trial: atezolizumab and chemotherapy in early relapsing metastatic triplenegative breast cancer. Future Oncol 2019;15:1951-61.

54 Adams S, Loi S, Toppmeyer D, et al. Pembrolizumab monotherapy for previously untreated, PD-L1-positive, metastatic triple-negative breast cancer: cohort B of the phase II KEYNOTE-086 study. Ann Oncol 2019;30:405-11.

55 Adams S, Schmid P, Rugo HS, et al. Pembrolizumab monotherapy for previously treated metastatic triple-negative breast cancer: cohort a of the phase II KEYNOTE-086 study. Ann Oncol 2019;30:397-404.

56 Adams S, Loi S, Toppmeyer D, et al. Phase 2 study of pembrolizumab as first-line therapy for PD-L1-positive metastatic triple-negative breast cancer (mTNBC): preliminary data from KEYNOTE-086 cohort B. Journal of Clinical Oncology 2017;35:1088.

57 Adams S, Schmid P, Rugo HS, et al. Phase 2 study of pembrolizumab (pembro) monotherapy for previously treated metastatic triple-negative breast cancer (mTNBC): KEYNOTE-086 cohort A. Journal of Clinical Oncology 2017;35:1008.

58 Cortés J, Lipatov O, Im S-A, et al. KEYNOTE-119: phase III study of pembrolizumab (pembro) versus single-agent chemotherapy (chemo) for metastatic triple negative breast cancer (mTNBC). Annals of Oncology 2019;30:v859-60.

59 Modi S, Saura C, Yamashita T, et al. Trastuzumab Deruxtecan in previously treated HER2-positive breast cancer. N Engl J Med Overseas Ed 2020;382:610-21.

60 Murthy RK, Loi S, Okines A, et al. Tucatinib, trastuzumab, and capecitabine for HER2-positive metastatic breast cancer. N Engl J Med Overseas Ed 2020;382:597-609.

61 Emens LA EF, Beresford M, Saura C. Results from KATE2, a randomized phase 2 study of atezolizumab (atezo)+ trastuzumab emtansine (T-DM1) vs placebo (pbo)+ T-DM1 in previously treated HER2+ advanced breast cancer (BC): SABCS. Cancer Res 2018;79.

62 Loi S, Giobbie-Hurder A, Gombos A, et al. Pembrolizumab plus trastuzumab in trastuzumab-resistant, advanced, HER2-positive breast cancer (PANACEA): a single-arm, multicentre, phase 1b-2 trial. Lancet Oncol 2019;20:371-82.

63 Dirix LY, Takacs I, Jerusalem G, et al. Avelumab, an anti-PD-L1 antibody, in patients with locally advanced or metastatic breast cancer: a phase 1B javelin solid tumor study. Breast Cancer Res Treat 2018;167:671-86.

64 Tolaney SM, Barroso-Sousa R, Keenan T, et al. Effect of eribulin with or without pembrolizumab on progression-free survival for patients with hormone receptor-positive, ERBB2-Negative metastatic breast cancer: a randomized clinical trial. JAMA Oncol 2020;6:1598-605

65 Nanda R, Liu MC, Yau C, et al. Effect of pembrolizumab plus neoadjuvant chemotherapy on pathologic complete response in women with early-stage breast cancer: an analysis of the ongoing phase 2 adaptively randomized I-SPY2 trial. JAMA Oncol 2020;6:676-84.

66 Liu MC, Robinson PA, Yau C, et al. Abstract P3-09-02: evaluation of a novel agent plus standard neoadjuvant therapy in early stage, high-risk HER2 negative breast cancer: results from the I-SPY 2 trial. Cancer Research 2020;80:P3-09-02-P3-09-02.

67 Schmid P, Park YH, Muñoz-Couselo E, et al. Abstract PD5-01: KEYNOTE-173: phase 1B multicohort study of pembrolizumab (Pembro) in combination with chemotherapy as neoadjuvant treatment for triple-negative breast cancer (TNBC). Cancer Research 2019;79:PD5-01-PD5-01.

68 Schmid P, Salgado R, Park YH, et al. Pembrolizumab plus chemotherapy as neoadjuvant treatment of high-risk, early-stage triple-negative breast cancer: results from the phase 1B open-label, multicohort KEYNOTE-173 study. Ann Oncol 2020;31:569-81.
69 Schmid P, Cortes J, Pusztai L, et al. Pembrolizumab for early triple-negative breast cancer. N Engl J Med Overseas Ed 2020;382:810-21

70 Schmid P, Cortes J, Dent R. KEYNOTE-522: phase 3 study of pembrolizumab (pembro) + chemotherapy (chemo) vs placebo (pbo) + chemo as neoadjuvant treatment, followed by pembro vs pbo as adjuvant treatment for early triple-negative breast cancer (TNBC), in ESMO 2019 Congress. Annals of Oncology 2019:v851-934.

71 Merck. KEYTRUDA® (pembrolizumab) KEYNOTE-522 -

Presentation to ODAC February 9, $20212021 \mathrm{https} / / / w w w . m e r c k$. $\mathrm{com} /$ news/fdas-oncologic-drugs-advisory-committee-to-discussmercks-application-for-keytruda-pembrolizumab-for-the-treatmentof-patients-with-high-risk-early-stage-triple-negative-brea-2/

72 Schmid P, Schmid P. KEYNOTE-522: phase III study of neoadjuvant pembrolizumab + chemotherapy vs. placebo + chemotherapy, followed by adjuvant pembrolizumab vs. placebo for early-stage TNBC. ESMO Virtual Plenary; 2021, Virtual, 2021.

73 Lajos Pusztai HSH, Yau C, Wolf D. Esserman, Trial Consortium I-SPY 2. CT011 - Evaluation of durvalumab in combination with olaparib and paclitaxel in high-risk HER2 negative stage II/III breast cancer: results from the I-SPY 2 TRIAL. In 111th Annual Meeting of the American Association for Cancer Research. 2020. Philadelphia, PA: AACR.

74 Loibl S, Untch M, Burchardi N, et al. A randomised phase II study investigating durvalumab in addition to an anthracycline taxanebased neoadjuvant therapy in early triple-negative breast cancer: clinical results and biomarker analysis of GeparNuevo study. Ann Oncol 2019;30:1279-88.

75 Gianni L, Han HS, Yau C, et al. Abstract GS3-04: pathologic complete response $(\mathrm{pCR})$ to neoadjuvant treatment with or without atezolizumab in triple negative, early high-risk and locally advanced breast cancer. NeoTRIPaPDL1 Michelangelo randomized study. Cancer Research 2020;80:GS3-04-GS3-04.

76 McArthur HL, Ignatiadis M, Guillaume S, et al. ALEXANDRA IMpassion030: a phase III study of standard adjuvant chemotherapy with or without atezolizumab in early-stage triple-negative breast cancer. Journal of Clinical Oncology 2019;37:TPS598.

77 Mittendorf EA, Zhang $\mathrm{H}$, Barrios $\mathrm{CH}$, et al. Neoadjuvant atezolizumab in combination with sequential nab-paclitaxel and anthracycline-based chemotherapy versus placebo and chemotherapy in patients with early-stage triple-negative breast cancer (IMpassion031): a randomised, double-blind, phase 3 trial. Lancet 2020;396:1090-100.

78 Geyer CE, Loibl S, Rastogi P, et al. Abstract OT2-04-08: a randomized double-blind phase III clinical trial of neoadjuvant chemotherapy (NAc) with atezolizumab or placebo in patients (PTS) with triple negative breast cancer (TNBC) followed by adjuvant atezolizumab or placebo: NSABP B-59/GBG 96-GeparDouze. Cancer Research 2020;80:OT2-04-08.

79 Cardoso F, Bardia A, Andre F, et al. KEYNOTE-756: randomized, double-blind, phase 3 study of pembrolizumab vs placebo combined with neoadjuvant chemotherapy and adjuvant endocrine therapy for high-risk, early-stage estrogen receptorpositive, human epidermal growth factor receptor 2-negative (ER+/HER2-) breast cancer. Journal of Clinical Oncology 2019;37:TPS601.

80 Loi S, McArthur H, Harbeck N, et al. Abstract OT2-04-03: nivolumab with neoadjuvant chemotherapy and adjuvant endocrine therapy in ER+/HER2- primary breast cancer: CheckMate 7FL. Cancer Research 2020;80:OT2-04-03-OT2-04-03.

81 Pusztai L, Barlow WE, Ganz PA, et al. Abstract OT1-02-04: SWOG S1418/NRG -BR006: A randomized, phase III trial to evaluate the efficacy and safety of MK-3475 as adjuvant therapy for triple receptor-negative breast cancer with $>1 \mathrm{~cm}$ residual invasive cancer or positive lymph nodes (>pN1 mic) after neoadjuvant chemotherapy. Cancer Research 2018;78:OT1-02.

82 Sørlie T, Perou CM, Tibshirani R, et al. Gene expression patterns of breast carcinomas distinguish tumor subclasses with clinical implications. Proc Natl Acad Sci U S A 2001;98:10869-74.

83 Sorlie T, Tibshirani R, Parker J, et al. Repeated observation of breast tumor subtypes in independent gene expression data sets. Proc Natl Acad Sci U S A 2003;100:8418-23.

84 Sotiriou C, Neo S-Y, McShane LM, et al. Breast cancer classification and prognosis based on gene expression profiles from a population-based study. Proc Natl Acad Sci U S A 2003:100:10393-8.

85 Lehmann BD, Bauer JA, Chen X, et al. Identification of human triple-negative breast cancer subtypes and preclinical models for selection of targeted therapies. J Clin Invest 2011;121:2750-67.

86 Giordano SH, Temin S, Chandarlapaty S, et al. Systemic therapy for patients with advanced human epidermal growth factor receptor 
2-positive breast cancer: ASCO clinical practice guideline update. $J$ Clin Oncol 2018;36:2736-40.

87 Burstein HJ, Lacchetti C, Anderson $\mathrm{H}$, et al. Adjuvant endocrine therapy for women with hormone receptor-positive breast cancer: ASCO clinical practice guideline focused update. J Clin Oncol 2019;37:423-38.

88 Allison $\mathrm{KH}$, Hammond $\mathrm{MEH}$, Dowsett $\mathrm{M}$, et al. Estrogen and progesterone receptor testing in breast cancer: ASCO/CAP guideline update. J Clin Oncol 2020;38:JCO.19.02309.

89 Wolff AC, Hammond MEH, Allison KH, et al. Human epidermal growth factor receptor 2 testing in breast cancer: American Society of clinical Oncology/College of American pathologists clinical practice guideline focused update. J Clin Oncol 2018;36:2105-22.

90 Robson M, Im S-A, Senkus E, et al. Olaparib for metastatic breas cancer in patients with a germline BRCA mutation. N Engl J Med Overseas Ed 2017;377:523-33.

91 Bardia A, Mayer IA, Vahdat LT, et al. Sacituzumab Govitecan-hziy in refractory metastatic triple-negative breast cancer. $N$ Engl $\mathrm{J}$ Med 2019;380:741-51.

92 Yates LR, Knappskog S, Wedge D, et al. Genomic evolution of breast cancer metastasis and relapse. Cancer Cell 2017;32:169-84.

93 Angus L, Smid M, Wilting SM, et al. The genomic landscape of metastatic breast cancer highlights changes in mutation and signature frequencies. Nat Genet 2019;51:1450-8.

94 Lindström LS, Karlsson E, Wilking UM, et al. Clinically used breast cancer markers such as estrogen receptor, progesterone receptor, and human epidermal growth factor receptor 2 are unstable throughout tumor progression. J Clin Oncol 2012;30:2601-8.

95 Macfarlane R, Seal M, Speers C, et al. Molecular alterations between the primary breast cancer and the subsequent locoregional/metastatic tumor. Oncologist 2012;17:172-8.

96 van de Ven S, Smit VTHBM, Dekker TJA, et al. Discordances in ER, PR and HER2 receptors after neoadjuvant chemotherapy in breast cancer. Cancer Treat Rev 2011;37:422-30.

97 André F, Bachelot T, Commo F, et al. Comparative genomic hybridisation array and DNA sequencing to direct treatment of metastatic breast cancer: a multicentre, prospective trial (SAFIR01/ UNICANCER). Lancet Oncol 2014;15:267-74.

98 Lefebvre C, Bachelot T, Filleron T, et al. Mutational profile of metastatic breast cancers: a retrospective analysis. PLoS Med 2016;13:e1002201.

99 Szekely B, Bossuyt V, Li X, et al. Immunological differences between primary and metastatic breast cancer. Ann Oncol 2018;29:2232-9.

100 Cimino-Mathews A, Ye X, Meeker A, et al. Metastatic triplenegative breast cancers at first relapse have fewer tumor-infiltrating lymphocytes than their matched primary breast tumors: a pilot study. Hum Pathol 2013;44:2055-63.

101 Li Y, Chang CW, Tran D, et al. Abstract PD6-01: prevalence of PDL1 and tumor infiltrating lymphocytes (TILs) in primary and metastatic TNBC. Cancer Research 2018;78:PD6-01.

102 Zheng M, Tian Z. Liver-Mediated adaptive immune tolerance. Front Immunol 2019:10:2525.

103 Lee JC, Mehdizadeh S, Smith J, et al. Regulatory T cell control of systemic immunity and immunotherapy response in liver metastasis. Sci Immunol 2020;5:eaba0759.

104 Narayan P, Wahby S, Gao JJ, et al. Fda approval summary: Atezolizumab plus paclitaxel protein-bound for the treatment of patients with advanced or metastatic TNBC whose tumors express PD-L1. Clin Cancer Res 2020;26:2284-9.

105 Hoda RS, Brogi E, Dos Anjos CH, et al. Clinical and pathologic features associated with PD-L1 (SP142) expression in stromal tumor-infiltrating immune cells of triple-negative breast carcinoma. Mod Pathol 2020;33:2221-32.

106 Thomas A, Routh ED, Pullikuth A, et al. Tumor mutational burden is a determinant of immune-mediated survival in breast cancer. Oncoimmunology 2018;7:e1490854.

$107 \mathrm{Xu} \mathrm{J}$, Bao H, Wu X, et al. Elevated tumor mutation burden and immunogenic activity in patients with hormone receptor-negative or human epidermal growth factor receptor 2-positive breast cancer. Oncol Lett 2019:18:449-55.

108 Lu S, Stein JE, Rimm DL, et al. Comparison of biomarker modalities for predicting response to PD-1/PD-L1 checkpoint blockade: a systematic review and meta-analysis. JAMA Oncol 2019;5:1195-204

109 Novartis. PIQRAY highlights of prescribing information, U. FDA, editor. Silver Spring, Maryland: Drugs@FDA, 2019.

110 Fong PC, Boss DS, Yap TA, et al. Inhibition of poly(ADP-ribose) polymerase in tumors from BRCA mutation carriers. N Engl J Med 2009;361:123-34.

111 Tutt A, Robson M, Garber JE, et al. Oral poly(ADP-ribose) polymerase inhibitor olaparib in patients with BRCA1 or BRCA2 mutations and advanced breast cancer: a proof-of-concept trial. Lancet 2010;376:235-44.

112 Przybytkowski E, Davis T, Hosny A, et al. An immune-centric exploration of BRCA1 and BRCA2 germline mutation related breast and ovarian cancers. BMC Cancer 2020;20:197.

113 Wen WX, Leong C-O. Association of BRCA1- and BRCA2deficiency with mutation burden, expression of PD-L1/PD-1, immune infiltrates, and T cell-inflamed signature in breast cancer. PLoS One 2019;14:e0215381.

114 Roy R, Chun J, Powell SN. Brca1 and BRCA2: different roles in a common pathway of genome protection. Nat Rev Cancer 2011:12:68-78.

115 Belli C, Duso BA, Ferraro E, et al. Homologous recombination deficiency in triple negative breast cancer. Breast 2019;45:15-21.

116 Lin $\mathrm{P}-\mathrm{H}$, Chen M, Tsai L-W, et al. Using next-generation sequencing to redefine BRCAness in triple-negative breast cancer. Cancer Sci 2020;111:1375-84

117 Taube JM, Galon J, Sholl LM, et al. Implications of the tumor immune microenvironment for staging and therapeutics. Mod Pathol 2018:31:214-34.

118 Walk EE, Yohe SL, Beckman A, et al. The cancer immunotherapy biomarker testing landscape. Arch Pathol Lab Med 2020;144:706-24

119 Li X, Li M, Lian Z, et al. Prognostic role of programmed death ligand-1 expression in breast cancer: a systematic review and meta-analysis. Target Oncol 2016;11:753-61.

120 Zhao T, Li C, Wu Y, et al. Prognostic value of PD-L1 expression in tumor infiltrating immune cells in cancers: a meta-analysis. PLOS One 2017;12:e0176822.

121 Mittendorf EA, Philips AV, Meric-Bernstam F, et al. Pd-L1 expression in triple-negative breast cancer. Cancer Immunol Res 2014;2:361-70.

122 Gaule P, Smithy JW, Toki M, et al. A quantitative comparison of antibodies to programmed cell death 1 ligand 1. JAMA Oncol 2017;3:256-9.

123 Sun WY, Lee YK, Koo JS. Expression of PD-L1 in triple-negative breast cancer based on different immunohistochemical antibodies. J Transl Med 2016;14:173.

124 O'Malley DP, Yang Y, Boisot S, et al. Immunohistochemical detection of PD-L1 among diverse human neoplasms in a reference laboratory: observations based upon 62,896 cases. Mod Pathol 2019;32:929-42.

125 Torlakovic E, Lim HJ, Adam J, et al. "Interchangeability" of PD-L1 immunohistochemistry assays: a meta-analysis of diagnostic accuracy. Mod Pathol 2020;33:4-17.

126 Lee SE, Park HY, Lim SD, et al. Concordance of programmed Death-Ligand 1 expression between SP142 and 22C3/SP263 assays in triple-negative breast cancer. $J$ Breast Cancer 2020:23:303-13.

127 U.S. Food \& Drug Administration (FDA). List of cleared or Approved companion diagnostic devices (in vitro and imaging tools. Silver Spring, Maryland: U.S. Food \& Drug Administration (FDA), 2020

128 Scheerens H, Malong A, Bassett K, et al. Current status of companion and complementary diagnostics: strategic considerations for development and Launch. Clin Trans/ Sci 2017; 10:84-92.

129 Cottrell TR, Taube JM. Pd-L1 and emerging biomarkers in immune checkpoint blockade therapy. Cancer J 2018;24:41-6.

130 Ventana medical systems, Inc. and Roche diagnostics international, Inc. Ventana PD-L1 (SP142 assay) interpretation guide for triple-negative breast carcinoma (TNBC. Oro Valley: Ventana medical systems, Inc. and Roche diagnostics international, Inc, 2019.

131 Rugo HS, Loi S, Adams S, et al. Performance of PD-L1 immunohistochemistry $(\mathrm{IHC})$ assays in unresectable locally advanced or metastatic triple-negative breast cancer (mTNBC): Post-hoc analysis of IMpassion130. Annals of Oncology 2019;30:v858-9.

132 Rugo H, Loi S, Adams S, et al. Abstract PD1-07: exploratory analytical harmonization of PD-L1 immunohistochemistry assays in advanced triple-negative breast cancer: a retrospective substudy of IMpassion130. Cancer Research 2020;80:PD1-07PD1-07.

133 Rimm DL, Han G, Taube JM, et al. Reanalysis of the NCCN PD-L1 companion diagnostic assay study for lung cancer in the context of PD-L1 expression findings in triple-negative breast cancer. Breast Cancer Res 2019;21:72.

134 Reisenbichler ES, Han G, Bellizzi A, et al. Prospective multiinstitutional evaluation of pathologist assessment of PD-L1 assays for patient selection in triple negative breast cancer. Mod Pathol 2020;33:1746-52. 
135 Jasani B, Bänfer G, Fish R, et al. Evaluation of an online training tool for scoring programmed cell death ligand-1 (PD-L1) diagnostic tests for lung cancer. Diagn Pathol 2020;15:37.

136 Martinez-Morilla S, McGuire J, Gaule P, et al. Quantitative assessment of PD-L1 as an analyte in immunohistochemistry diagnostic assays using a standardized cell line tissue microarray. Lab Invest 2020;100:4-15.

137 Vennapusa B, Baker B, Kowanetz M, et al. Development of a PD-L1 complementary diagnostic immunohistochemistry assay (SP142) for Atezolizumab. Appl Immunohistochem Mol Morphol 2019;27:92-100.

138 Malhotra MK, Emens LA. The evolving management of metastatic triple negative breast cancer. Semin Oncol 2020;47:229-37.

139 Schmid P, Cortes J, Pusztai L, et al. Pembrolizumab for early triplenegative breast cancer. N Engl J Med 2020;382:810-21.

140 Loi S, Giobbie-Hurder A, Gombos A, et al. Pembrolizumab plus trastuzumab in trastuzumab-resistant, advanced, HER2-positive breast cancer (panacea): a single-arm, multicentre, phase $1 \mathrm{~b}-2$ trial. Lancet Oncol 2019;20:371-82.

141 Chia S, Bedard PL, Hilton J, et al. A phase lb trial of Durvalumab in combination with trastuzumab in HER2-positive metastatic breast cancer (Cctg IND.229). Oncologist 2019;24:1439-45.

142 Lee J-M, Cimino-Mathews A, Peer CJ, et al. Safety and clinical activity of the programmed Death-Ligand 1 inhibitor Durvalumab in combination with poly (ADP-ribose) polymerase inhibitor olaparib or vascular endothelial growth factor receptor 1-3 inhibitor cediranib in women's cancers: a dose-escalation, phase I study. J Clin Oncol 2017;35:2193-202.

143 Emens LA, Loi S, Rugo HS, et al. IMpassion130: efficacy in immune biomarker subgroups from the global, randomized, double-blind, placebo-controlled, phase III study of atezolizumab+ nab-paclitaxe in patients with treatment-naïve, locally advanced or metastatic triple-negative breast cancer. In San Antonio Breast Cancer Symposium 2018;5.

144 Emens LA, Cruz C, Eder JP, et al. Long-Term clinical outcomes and biomarker analyses of Atezolizumab therapy for patients with metastatic triple-negative breast cancer: a phase 1 study. JAMA Oncol 2019;5:74-82.

145 Salgado R, Denkert C, Demaria S, et al. The evaluation of tumorinfiltrating lymphocytes (TILs) in breast cancer: recommendations by an international TILs Working group 2014. Ann Oncol 2015;26:259-71.

146 Lokuhetty Det al. Who classification of breast tumours. Geneva: WHO, 2019

147 Tan PH, Ellis I, Allison K, et al. The 2019 World Health Organization classification of tumours of the breast. Histopathology 2020;77:181-5

148 O'Loughlin M, Andreu X, Bianchi S, et al. Reproducibility and predictive value of scoring stromal tumour infiltrating lymphocytes in triple-negative breast cancer: a multi-institutional study. Breast Cancer Res Treat 2018;171:1-9.

149 Adams S, Gray RJ, Demaria S, et al. Prognostic value of tumorinfiltrating lymphocytes in triple-negative breast cancers from two phase III randomized adjuvant breast cancer trials: ECoG 2197 and ECoG 1199. J Clin Oncol 2014;32:2959-66. doi:10.1200/ JCO.2013.55.0491

150 Loi S, Michiels S, Salgado R, et al. Tumor infiltrating lymphocytes are prognostic in triple negative breast cancer and predictive for trastuzumab benefit in early breast cancer: results from the FinHER trial. Ann Oncol 2014;25:1544-50.

151 Loi S, Sirtaine N, Piette F, et al. Prognostic and predictive value of tumor-infiltrating lymphocytes in a phase III randomized adjuvant breast cancer trial in node-positive breast cancer comparing the addition of docetaxel to doxorubicin with doxorubicin-based chemotherapy: big 02-98. J Clin Oncol 2013;31:860-7.

152 Denkert C, von Minckwitz G, Darb-Esfahani S, et al. TumourInfiltrating lymphocytes and prognosis in different subtypes of breast cancer: a pooled analysis of 3771 patients treated with neoadjuvant therapy. Lancet Oncol 2018;19:40-50.

153 Criscitiello C, Vingiani A, Maisonneuve P, et al. Tumor-Infiltrating lymphocytes (TILs) in ER+/HER2- breast cancer. Breast Cancer Res Treat 2020;183:347-54.

154 Gruosso T, Gigoux M, Manem VSK, et al. Spatially distinct tumor immune microenvironments stratify triple-negative breast cancers. $J$ Clin Invest 2019;129:1785-800.

155 Mani NL, Schalper KA, Hatzis C, et al. Quantitative assessment of the spatial heterogeneity of tumor-infiltrating lymphocytes in breast cancer. Breast Cancer Res 2016;18:78.

156 Savas P, Virassamy B, Ye C, et al. Single-Cell profiling of breast cancer $T$ cells reveals a tissue-resident memory subset associated with improved prognosis. Nat Med 2018;24:986-93.
157 Heindl A, Sestak I, Naidoo K, et al. Relevance of spatial heterogeneity of immune infiltration for predicting risk of recurrence after endocrine therapy of ER+ breast cancer. J Natl Cancer Inst 2018;110:166-75.

158 Guo L, Li W, Zhu X, et al. Pd-L1 expression and CD274 gene alteration in triple-negative breast cancer: implication for prognostic biomarker. Springerplus 2016;5:805.

159 Goodman AM, Piccioni D, Kato S, et al. Prevalence of PDL1 amplification and preliminary response to immune checkpoint blockade in solid tumors. JAMA Oncol 2018;4:1237-44.

160 Barrett MT, Anderson KS, Lenkiewicz E, et al. Genomic amplification of 9p24.1 targeting JAK2, PD-L1, and PD-L2 is enriched in high-risk triple negative breast cancer. Oncotarget 2015;6:26483-93.

161 Balko JM, Schwarz LJ, Luo N, et al. Triple-Negative breast cancers with amplification of JAK2 at the 9p24 locus demonstrate JAK2specific dependence. Sci Transl Med 2016;8:334ra53.

162 Bachelot T, Filleron T, Dalenc F, et al. 1280 PDL1/CD274 gain/ amplification as a predictive marker of checkpoint blockade inhibitor efficacy in metastatic breast cancer: Exploratory analysis of the SAFIR02-IMMUNO randomized phase II trial. Annals of Oncology 2020;31:S58-9.

163 Ayers M, Lunceford J, Nebozhyn M, et al. IFN- $\gamma$-related mRNA profile predicts clinical response to PD-1 blockade. J Clin Invest 2017:127:2930-40.

164 Vafaizadeh V, Barekati Z. Immuno-Oncology biomarkers for personalized immunotherapy in breast cancer. Front Cell Dev Biol 2020;8:162.

165 Organization, W.H. WHO Handbook for reporting results of cancer treatment. Geneva: World Health Organization, 1979.

166 Therasse P, Arbuck SG, Eisenhauer EA, et al. New guidelines to evaluate the response to treatment in solid tumors. $J$ Natl Cancer Inst 2000:92:205-16.

167 Queirolo P, Spagnolo F. Atypical responses in patients with advanced melanoma, lung cancer, renal-cell carcinoma and other solid tumors treated with anti-PD-1 drugs: a systematic review. Cancer Treat Rev 2017;59:71-8.

168 Tumeh PC, Radu CG, Ribas A. Pet imaging of cancer immunotherapy. J Nucl Med 2008;49:865-8.

169 Dromain C, Beigelman C, Pozzessere C, et al. Imaging of tumour response to immunotherapy. Eur Radiol Exp 2020;4:2.

170 Kurra V, Sullivan RJ, Gainor JF, et al. Pseudoprogression in cancer immunotherapy: rates, time course and patient outcomes. Journal of Clinical Oncology 2016;34:6580.

171 Wolchok JD, Hoos A, O'Day S, et al. Guidelines for the evaluation of immune therapy activity in solid tumors: immune-related response criteria. Clin Cancer Res 2009;15:7412-20.

172 Nishino M, Giobbie-Hurder A, Gargano M, et al. Developing a common language for tumor response to immunotherapy: immunerelated response criteria using unidimensional measurements. Clin Cancer Res 2013;19:3936-43.

173 Hodi FS, Ballinger M, Lyons B, et al. Immune-modified response evaluation criteria in solid tumors (imRECIST): refining guidelines to assess the clinical benefit of cancer immunotherapy. J Clin Oncol 2018;36:850-8.

174 Seymour L, Bogaerts J, Perrone A, et al. iRECIST: guidelines for response criteria for use in trials testing immunotherapeutics. Lancet Oncol 2017;18:e143-52.

175 Nishino M, Gargano M, Suda M, et al. Optimizing immune-related tumor response assessment: does reducing the number of lesions impact response assessment in melanoma patients treated with ipilimumab? J Immunother Cancer 2014:2:17.

176 Mulkey F, Theoret MR, Keegan P, et al. Comparison of iRECIST versus RECIST V.1.1 in patients treated with an anti-PD-1 or PD-L1 antibody: pooled FDA analysis. J Immunother Cancer 2020;8:e000146.

177 Stein JE, Lipson EJ, Cottrell TR, et al. Pan-tumor pathologic scoring of response to PD-(L)1 blockade. Clin Cancer Res 2020;26:545-51.

178 Cottrell TR, Thompson ED, Forde PM, et al. Pathologic features of response to neoadjuvant anti-PD-1 in resected non-small-cell lung carcinoma: a proposal for quantitative immune-related pathologic response criteria (irPRC). Ann Oncol 2018;29:1853-60.

179 Okada $\mathrm{H}$, Weller $\mathrm{M}$, Huang $\mathrm{R}$, et al. Immunotherapy response assessment in neuro-oncology: a report of the RANO Working group. Lancet Oncol 2015;16:e534-42.

180 Kuczynski EA, Sargent DJ, Grothey A, et al. Drug rechallenge and treatment beyond progression--implications for drug resistance. Nat Rev Clin Oncol 2013;10:571-87.

181 Palmieri C, Krell J, James CR, et al. Rechallenging with anthracyclines and taxanes in metastatic breast cancer. Nat Rev Clin Oncol 2010;7:561-74. 
182 Cara S, Tannock IF. Retreatment of patients with the same chemotherapy: implications for clinical mechanisms of drug resistance. Ann Oncol 2001;12:23-7.

183 GBG GERMAN BREAST GROUP, Pirvulescu C, Uhlig M, et al Trastuzumab improves the efficacy of chemotherapy in breast cancer treatment beyond progression. Breast Care 2008;3:364-5.

184 Park IH, Ro J, Lee KS, et al. Trastuzumab treatment beyond brain progression in HER2-positive metastatic breast cancer. Ann Oncol 2009;20:56-62.

185 Tsimberidou AM, Levit LA, Schilsky RL, et al. Trial reporting in Immuno-Oncology (trio): an American Society of clinical OncologySociety for immunotherapy of cancer statement. J Clin Oncol 2019;37:72-80.

186 Deutsch GB, Flaherty DC, Kirchoff DD, et al. Association of surgical treatment, systemic therapy, and survival in patients with abdominal visceral melanoma metastases, 1965-2014: relevance of surgical cure in the era of modern systemic therapy. JAMA Surg 2017;152:672-8.

187 Flaherty DC, Deutsch GB, Kirchoff DD, et al. Adrenalectomy for metastatic melanoma: current role in the age of nonsurgical treatments. Am Surg 2015;81:1005-9.

188 Fife KM, Colman MH, Stevens GN, et al. Determinants of outcome in melanoma patients with cerebral metastases. J Clin Oncol 2004:22:1293-300.

189 Bale R, Putzer D, Schullian P. Local treatment of breast cancer liver metastasis. Cancers 2019;11:1341.

190 Reynders K, Illidge T, Siva S, et al. The abscopal effect of local radiotherapy: using immunotherapy to make a rare event clinically relevant. Cancer Treat Rev 2015;41:503-10.

191 Barroso-Sousa R, Krop IE, Trippa L, et al. A phase II study of pembrolizumab in combination with palliative radiotherapy for hormone receptor-positive metastatic breast cancer. Clin Breast Cancer 2020;20:238-45.

192 Robert C, Marabelle A, Herrscher H, et al. Immunotherapy discontinuation - how, and when? Data from melanoma as a paradigm. Nat Rev Clin Oncol 2020;17:707-15.

193 Adams S, Diamond J, Hamilton E, et al. Safety and clinical activity of atezolizumab (anti-PDL1) in combination with nab-paclitaxel in patients with metastatic triple-negative breast cancer. In Proceedings of the Thirty-Eighth Annual CTRC-AACR San Antonio Breast Cancer Symposium 2015:8-12.

194 Page DB, Bear H, Prabhakaran S, et al. Two may be better than one: PD-1/PD-L1 blockade combination approaches in metastatic breast cancer. NPJ Breast Cancer 2019;5:34

195 Goedert JJ, Schairer C, McNeel TS, et al. Risk of breast, ovary, and uterine corpus cancers among 85,268 women with AIDS. Br J Cancer 2006;95:642-8.

196 Franceschi S, Lise M, Clifford GM, et al. Changing patterns of cancer incidence in the early- and late-HAART periods: the Swiss HIV cohort study. Br J Cancer 2010;103:416-22.

197 Coghill AE, Shiels MS, Suneja G, et al. Elevated cancer-specific mortality among HIV-infected patients in the United States. J Clin Oncol 2015;33:2376-83.

198 Hessol NA, Whittemore H, Vittinghoff E, et al. Incidence of first and second primary cancers diagnosed among people with HIV 1985-2013: a population-based, registry linkage study. Lancet HIV 2018;5:e647-55.

199 Cook MR, Kim C. Safety and efficacy of immune checkpoint inhibitor therapy in patients with HIV infection and advanced-stage cancer. JAMA Oncol 2019;5:1049-54.

200 González-Cao M, Moran T, Dalmau J, et al. Phase II study of durvalumab (MEDI4736) in cancer patients HIV-1-infected. Journal of Clinical Oncology 2019;37:2501.

201 Uldrick TS, Gonçalves PH, Abdul-Hay M, et al. Assessment of the safety of pembrolizumab in patients with HIV and advanced cancer-a phase 1 study. JAMA Oncol 2019;5:1332-9.

202 Menzies AM, Johnson DB, Ramanujam S, et al. Anti-Pd-1 therapy in patients with advanced melanoma and preexisting autoimmune disorders or major toxicity with ipilimumab. Ann Oncol 2017;28:368-76.

203 van Holstein Y, Kapiteijn E, Bastiaannet E, et al. Efficacy and adverse events of immunotherapy with checkpoint inhibitors in older patients with cancer. Drugs Aging 2019;36:927-38.

204 Sattar J, Kartolo A, Hopman WM, et al. The efficacy and toxicity of immune checkpoint inhibitors in a real-world older patient population. J Geriatr Oncol 2019;10:411-4

205 Kanesvaran R, Cordoba R, Maggiore R. Immunotherapy in older adults with advanced cancers: implications for clinical decisionmaking and future research. Am Soc Clin Oncol Educ Book 2018:38:400-14.
206 Arbour KC, Mezquita L, Long N, et al. Impact of baseline steroids on efficacy of programmed cell death-1 and programmed DeathLigand 1 blockade in patients with non-small-cell lung cancer. J Clin Oncol 2018;36:2872-8.

207 Scott SC, Pennell NA. Early use of systemic corticosteroids in patients with advanced NSCLC treated with nivolumab. J Thorac Oncol 2018:13:1771-5.

208 Ricciuti B, Dahlberg SE, Adeni A, et al. Immune checkpoint inhibitor outcomes for patients with non-small-cell lung cancer receiving baseline corticosteroids for palliative versus Nonpalliative indications. J Clin Oncol 2019;37:1927-34.

209 Vaidya JS, Baldassarre G, Thorat MA, et al. Role of glucocorticoids in breast cancer. Curr Pharm Des 2010;16:3593-600.

210 Moutsatsou P, Papavassiliou AG. The glucocorticoid receptor signalling in breast cancer. J Cell Mol Med 2008;12:145-63.

211 Petrelli F, Signorelli D, Ghidini M, et al. Association of steroids use with survival in patients treated with immune checkpoint inhibitors: a systematic review and meta-analysis. Cancers 2020;12. doi:10.3390/cancers12030546. [Epub ahead of print: 2702 2020].

212 Wong G, Au E, Badve SV, et al. Breast cancer and transplantation. Am J Transplant 2017;17:2243-53.

213 Abdel-Wahab N, Safa H, Abudayyeh A, et al. Checkpoint inhibitor therapy for cancer in solid organ transplantation recipients: an institutional experience and a systematic review of the literature. $J$ Immunother Cancer 2019;7:106.

214 D'Abreo N, Adams S. Immune-checkpoint inhibition for metastatic triple-negative breast cancer: safety first? Nat Rev Clin Oncol 2019;16:399-400.

215 Puzanov I, Diab A, Abdallah K, et al. Managing toxicities associated with immune checkpoint inhibitors: consensus recommendations from the Society for immunotherapy of cancer (SITC) toxicity management Working group. J Immunother Cancer 2017;5:95.

216 Pallin DJ, Baugh CW, Postow MA, et al. Immune-Related adverse events in cancer patients. Acad Emerg Med 2018;25:819-27.

217 Brahmer JR, Lacchetti C, Schneider BJ, et al. Management of immune-related adverse events in patients treated with immune checkpoint inhibitor therapy: American Society of clinical oncology clinical practice guideline. J Clin Oncol 2018;36:1714-68.

218 Myers G. Immune-Related adverse events of immune checkpoint inhibitors: a brief review. Curr Oncol 2018;25:342-7.

219 Weber JS, Hodi FS, Wolchok JD, et al. Safety profile of nivolumab monotherapy: a pooled analysis of patients with advanced melanoma. J Clin Oncol 2017;35:785-92.

220 Brahmer JR, Abu-Sbeih H, Ascierto PA, et al. Society for immunotherapy of cancer (SITC) clinical practice guideline on immune checkpoint inhibitor-related adverse events. J Immunother Cancer 2021;9:e002435.

221 Barroso-Sousa R, Barry WT, Garrido-Castro AC, et al. Incidence of endocrine dysfunction following the use of different immune checkpoint inhibitor regimens: a systematic review and metaanalysis. JAMA Oncol 2018;4:173-82.

222 Quandt Z, Trupin L, Evans M, et al. SAT-418 finding the needles in the haystack: harnessing the electronic health record to find thyroid immune related adverse events. J Endocr Soc 2020;4:SAT-418.

223 Zhai Y, Ye X, Hu F, et al. Endocrine toxicity of immune checkpoint inhibitors: a real-world study Leveraging US food and drug administration adverse events reporting system. J Immunother Cancer 2019;7:286

224 Chen TW, Razak AR, Bedard PL, et al. A systematic review of immune-related adverse event reporting in clinical trials of immune checkpoint inhibitors. Ann Oncol 2015;26:1824-9.

225 Couey MA, Bell RB, Patel AA, et al. Delayed immune-related events (dire) after discontinuation of immunotherapy: diagnostic hazard of autoimmunity at a distance. J Immunother Cancer 2019;7:165.

226 Simonaggio A, Michot JM, Voisin AL, et al. Evaluation of readministration of immune checkpoint inhibitors after immunerelated adverse events in patients with cancer. JAMA Oncol 2019:5:1310-7.

227 Abu-Sbeih H, Ali FS, Naqash AR, et al. Resumption of immune checkpoint inhibitor therapy after immune-mediated colitis. J Clin Oncol 2019;37:2738-45

228 Das S, Johnson DB. Immune-Related adverse events and antitumor efficacy of immune checkpoint inhibitors. $J$ Immunother Cancer 2019;7:306.

229 Mittendorf E, Barrios $\mathrm{CH}$, Harbeck N, et al. Abstract OT207-03: IMpassion031: a phase III study comparing neoadjuvant atezolizumab vs placebo in combination with nab-paclitaxel-based chemotherapy in early triple-negative breast cancer (TNBC). Cancer Research 2018;78.

230 Louvel G, Bahleda R, Ammari S, et al. Immunotherapy and pulmonary toxicities: can concomitant immune-checkpoint 
inhibitors with radiotherapy increase the risk of radiation pneumonitis? Eur Respir J 2018;51:1701737.

231 Schoenfeld JD, Nishino M, Severgnini M, et al. Pneumonitis resulting from radiation and immune checkpoint blockade illustrates characteristic clinical, radiologic and circulating biomarker features. $J$ Immunother Cancer 2019;7:112.

232 Cooksley CD, Avritscher EBC, Bekele BN, et al. Epidemiology and outcomes of serious influenza-related infections in the cancer population. Cancer 2005;104:618-28.

233 Kunisaki KM, Janoff EN. Influenza in immunosuppressed populations: a review of infection frequency, morbidity, mortality, and vaccine responses. Lancet Infect Dis 2009;9:493-504.

234 Gwynn ME, DeRemer DL, Saunders KM, et al. Immune-Mediated adverse events following influenza vaccine in cancer patients receiving immune checkpoint inhibitors. J Oncol Pharm Pract 2020;26:647-54.

235 Chong CR, Park VJ, Cohen B, et al. Safety of inactivated influenza vaccine in cancer patients receiving immune checkpoint inhibitors. Clin Infect Dis 2020;70:193-9.

236 Shah MK, Kamboj M. Immunizing cancer patients: which patients? which vaccines? when to give? Oncology 2018;32:254-8.

237 Rubin KMMelanoma DE, Bastian BC, eds. Evolving role of the oncology nurse in the care of patients with melanoma. Springer New York: New York, NY, 2019: 1-27.

238 Ganz PA, Coscarelli A, Fred C, et al. Breast cancer survivors: psychosocial concerns and quality of life. Breast Cancer Res Treat 1996;38:183-99.

239 Mehmood T. Quality of life and psychosocial needs of metastatic breast cancer patients. Annals of Oncology 2018;29:ix19.

240 Adams S, Diéras V, Barrios CH, et al. Patient-Reported outcomes from the phase III IMpassion130 trial of atezolizumab plus nabpaclitaxel in metastatic triple-negative breast cancer. Ann Onco 2020;31:582-9.

241 Dineen R, Thompson CJ, Sherlock M. Adrenal crisis: prevention and management in adult patients. Ther Adv Endocrinol Metab 2019;10:2042018819848218.

242 Duma N, Lambertini M. It is time to talk about fertility and immunotherapy. Oncologist 2020;25:277-8.

243 Mehta A, Kim KB, Minor DR. Case report of a pregnancy during ipilimumab therapy. J Glob Oncol 2018;4:1-3.

244 Bucheit AD, Hardy JT, Szender JB, et al. Conception and viable twin pregnancy in a patient with metastatic melanoma while treated with CTLA-4 and PD-1 checkpoint inhibition. Melanoma Res 2020;30:423-5.

245 Woodruff TK. Oncofertility: a grand collaboration between reproductive medicine and oncology. Reproduction 2015;150:S1-10

246 Faje A. Immunotherapy and hypophysitis: clinical presentation, treatment, and biologic insights. Pituitary 2016;19:82-92.

247 Food and Drug Administration, HHS. Content and format of labeling for human prescription drug and biological products; requirements for pregnancy and lactation labeling. final rule. Fed Regist 2014;79:72063-103.

248 Coscia LA, Armenti DP, King RW, et al. Update on the teratogenicity of maternal mycophenolate mofetil. J Pediatr Genet 2015;4:42-55.

249 Ponticelli C, Moroni G. Fetal toxicity of immunosuppressive drugs in pregnancy. J Clin Med 2018;7:552.

250 Ferreira AR, Di Meglio A, Pistilli B, et al. Differential impact of endocrine therapy and chemotherapy on quality of life of breast cancer survivors: a prospective patient-reported outcomes analysis. Ann Oncol 2019;30:1784-95.

251 Osoba D, Slamon DJ, Burchmore M, et al. Effects on quality of life of combined trastuzumab and chemotherapy in women with metastatic breast cancer. J Clin Oncol 2002;20:3106-13.

252 Hwang SY, Chang SJ, Park B-W. Does chemotherapy really affect the quality of life of women with breast cancer? J Breast Cancer 2013;16:229-35.

253 Sharma N, Purkayastha A. Factors affecting quality of life in breast cancer patients: a descriptive and cross-sectional study with review of literature. J Midlife Health 2017:8:75-83.

254 Perry S, Kowalski TL, Chang C-H. Quality of life assessment in women with breast cancer: benefits, acceptability and utilization. Health Qual Life Outcomes 2007:5:24.

255 Avis NE, Crawford S, Manuel J. Quality of life among younger women with breast cancer. J Clin Oncol 2005;23:3322-30.

256 Marschner N, Trarbach T, Rauh J, et al. Quality of life in pre- and postmenopausal patients with early breast cancer: a comprehensive analysis from the prospective MaLife project. Breast Cancer Res Treat 2019;175:701-12

257 Napolitano S, Brancaccio G, Argenziano G, et al. It is finally time for adjuvant therapy in melanoma. Cancer Treat Rev 2018;69:101-11.
258 Mittendorf EA, Hong Zhang NH, Saji S, et al. Barrios, Patientreported outcomes (PROs) from the Ph 3 IMpassion031 trial of neoadjuvant (NA) atezolizumab + chemo in early triple-negative breast cancer(eTNBC). In 2020 San Antonio Breast Cancer Symposium 2020

259 Kang C, Syed YY, Syed, Atezolizumab YY. Atezolizumab (in combination with nab-paclitaxel): a review in advanced triplenegative breast cancer. Drugs 2020;80:601-7.

260 Schmid P, Haiderali A, Mejia J, et al. 141P impact of pembrolizumab versus chemotherapy on health-related quality of life in patients with metastatic triple negative breast cancer. Annals of Oncology 2020;31:S65-6.

261 Faury S, Foucaud J. Health-Related quality of life in cancer patients treated with immune checkpoint inhibitors: a systematic review on reporting of methods in randomized controlled trials. PLoS One 2020;15:e0227344.

262 Tsoutsou PG, Zaman K, Martin Lluesma S, et al. Emerging opportunities of radiotherapy combined with immunotherapy in the era of breast cancer heterogeneity. Front Oncol 2018;8:609.

263 Ho AY, Barker CA, Arnold BB, et al. A phase 2 clinical trial assessing the efficacy and safety of pembrolizumab and radiotherapy in patients with metastatic triple-negative breast cancer. Cancer 2020;126:850-60.

264 Ho AY, Wright JL, Blitzblau RC, et al. Optimizing radiation therapy to boost systemic immune responses in breast cancer: a critical review for breast radiation oncologists. Int J Radiat Oncol Biol Phys 2020;108:227-41.

265 McArthur HL, Diab A, Page DB, et al. A pilot study of preoperative single-dose ipilimumab and/or cryoablation in women with earlystage breast cancer with comprehensive immune profiling. Clin Cancer Res 2016;22:5729-37.

266 Vikas P, Borcherding N, Chennamadhavuni A, et al. Therapeutic potential of combining PARP inhibitor and immunotherapy in solid tumors. Front Oncol 2020;10:570.

267 Vinayak S, Tolaney SM, Schwartzberg LS, et al. TOPACIO/ Keynote-162: Niraparib + pembrolizumab in patients (PTS) with metastatic triple-negative breast cancer (TNBC), a phase 2 trial. Journal of Clinical Oncology 2018;36:1011.

268 Domchek S, Postel-Vinay S, Im S-A, et al. Phase II study of olaparib (o) and durvalumab (D) (MEDIOLA): updated results in patients (PTS) with germline BRCA-mutated (gBRCAm) metastatic breast cancer (MBC). Annals of Oncology 2019;30:v477.

269 Yukinori Ozaki TM, Tsurutani J, Takahashi M. A multicenter phase II study evaluating the efficacy of nivolumab plus paclitaxel plus bevacizumab triple-combination therapy as a first-line treatment in patients with HER2-negative metastatic breast cancer: WJOG9917B NEWBEAT trial. In San Antonio Breast Cancer Symposium 2019: San Antonio, TX 2019.

270 Goel S, DeCristo MJ, Watt AC, et al. Cdk4/6 inhibition triggers antitumour immunity. Nature 2017;548:471-5.

271 Tolaney SM, Kabos P, Dickler MN, et al. Updated efficacy, safety, \& PD-L1 status of patients with HR+, HER2- metastatic breast cance administered abemaciclib plus pembrolizumab. Journal of Clinical Oncology 2018;36:1059.

272 Rugo HS, Beck JT, Jerusalem G, et al. Abstract CT108: a phase $1 \mathrm{~B}$ study of abemaciclib in combination with pembrolizumab for patients (PTS) with hormone receptor positive $(\mathrm{HR}+)$, human epidermal growth factor receptor 2 negative (HER2-) metastatic breast cancer (mBC) (NCT02779751): preliminary results. Cancer Research 2020;80:CT108.

273 Masuda J, Tsurutani J, Masuda N, et al. Abstract OT2-04-07: phase II study of nivolumab in combination with abemaciclib plus endocrine therapy in patients with hormone receptor-positive, human epidermal growth factor receptor-2 negative metastatic breast cancer (WJOG11418B, NEWFLAME trial). Cancer Research 2020;80:OT2-04-07.

274 Masuda JTJ, Masuda N, Tsurutani J, et al. Phase II study of nivolumab in combination with abemaciclib plus endocrine therapy in patients with HR+, HER2- metastatic breast cancer: WJOG11418B NEWFLAME trial. In 2020 Virtual San Antonio Breast Cancer Symposium 2020.

275 Tolaney SM, Jerusalem G, Salgado R, et al. A phase II trial of nivolumab (NIVO) + palbociclib (PAL) + anastrozole (ANA) in postmenopausal women and men with estrogen receptor (ER)+/human epidermal growth factor 2 (HER2)- primary breast cancer (BC): CheckMate 7A8. Journal of Clinical Oncology 2020;38:TPS1105.

276 Madjd Z, Spendlove I, Pinder SE, et al. Total loss of MHC class I is an independent indicator of good prognosis in breast cancer. Int $J$ Cancer 2005;117:248-55. 
277 Park HS, Cho U, Im SY, et al. Loss of human leukocyte antigen class I expression is associated with poor prognosis in patients with advanced breast cancer. J Pathol Trans/ Med 2019;53:75-85.

278 Inoue M, Mimura K, Izawa S, et al. Expression of MHC class I on breast cancer cells correlates inversely with HER2 expression. Oncoimmunology 2012;1:1104-10.

279 Piha-Paul S, Bendell J, Tolcher A, et al. O82 A phase 1 dose escalation study of PRS-343, a HER2/4-1BB bispecific molecule, in patients with HER2-positive malignancies. $J$ Immunother Cancer 2020;8:A1.2-2.

280 Leone RD, Emens LA. Targeting adenosine for cancer immunotherapy. J Immunother Cancer 2018;6:57.

281 Gandhi N, Das GM. Metabolic reprogramming in breast cancer and its therapeutic implications. Cells 2019;8:89.

282 Gang BP, Dilda PJ, Hogg PJ, et al. Targeting of two aspects of metabolism in breast cancer treatment. Cancer Biol Ther 2014;15:1533-41.

283 Beavis PA, Divisekera U, Paget C, et al. Blockade of A2A receptors potently suppresses the metastasis of CD73+ tumors. Proc Natl Acad Sci U S A 2013:110:14711-6.

284 Chen G, Gupta R, Petrik S, et al. A feasibility study of cyclophosphamide, trastuzumab, and an allogeneic GM-CSF- secreting breast tumor vaccine for HER2+ metastatic breast cancer. Cancer Immunol Res 2014;2:949-61.

285 Disis ML, Wallace DR, Gooley TA, et al. Concurrent trastuzumab and HER2/neu-specific vaccination in patients with metastatic breast cancer. J Clin Oncol 2009;27:4685-92.

286 Miles D, Roché $\mathrm{H}$, Martin M, et al. Phase III multicenter clinical trial of the sialyl-TN (STn)-keyhole limpet hemocyanin $(\mathrm{KLH})$ vaccine for metastatic breast cancer. Oncologist 2011;16:1092-100.

287 Vonderheide RH, LoRusso PM, Khalil M, et al. Tremelimumab in combination with exemestane in patients with advanced breast cancer and treatment-associated modulation of inducible costimulator expression on patient T cells. Clin Cancer Res 2010;16:3485-94.

288 Kataoka Y, Hirano K. Which criteria should we use to evaluate the efficacy of immune-checkpoint inhibitors? Ann Transl Med 2018;6:222.

289 Eisenhauer EA, Therasse P, Bogaerts J, et al. New response evaluation criteria in solid tumours: revised RECIST guideline (version 1.1). Eur J Cancer 2009;45:228-47. 


\section{Correction: Society for Immunotherapy of Cancer (SITC) clinical practice guideline on immunotherapy for the treatment of breast cancer}

Emens LA, Adams S, Cimino-Mathews A, et al. Society for Immunotherapy of Cancer (SITC) clinical practice guideline on immunotherapy for the treatment of breast cancer. J ImmunoTher Cancer 2021;9:e002597. doi: 10.1136/jitc-2021-002597

\section{UPDATE TO THE SITC GUIDELINE ON BREAST CANCER ADDRESSING WITHDRAWAL OF ATEZOLIZUMAB INDICATION FOR ADVANCED TNBC}

On August 27, 2021, the indication for atezolizumab in combination with nab-paclitaxel as treatment for patients with triple-negative breast cancer (TNBC) whose tumors express PD-L1 was voluntarily withdrawn by the manufacturer. According to the press release announcing the withdrawal, the decision was not related to any changes in either the efficacy or safety associated with atezolizumab but rather to recent changes in the treatment landscape for TNBC.

In light of the withdrawal, 'Society for Immunotherapy of Cancer (SITC) clinical practice guideline on immunotherapy for the treatment of breast cancer' has been updated. The following changes have been made to the manuscript. Amendments to the original text are shown in italics. The amendments below are grouped by the sections in which they appear in the order of the original publication.

\section{Introduction}

The following sentences have been modified to include information on the withdrawal of atezolizumab: 'In 2019, the United States (US) Food and Drug Administration (FDA) granted accelerated approval of the PD-L1-directed antibody, atezolizumab, in combination with nanoparticle albumin-bound (nab)-paclitaxel for advanced/metastatic PD-L1-positive (PD-L1+) TNBC, ${ }^{17}$ based on the results of the phase III IMpassion130 trial. Furthermore, in 2020, the FDA granted accelerated approval to the PD-1-directed antibody, pembrolizumab, in combination with chemotherapy for advanced/metastatic PD-L1+ TNBC based on the results of the phase III KEYNOTE-355 trial.'

The updated text now reads: 'In 2019, the United States (US) Food and Drug Administration (FDA) granted accelerated approval of the PD-L1-directed antibody, atezolizumab, in combination with nanoparticle albumin-bound (nab)-paclitaxel for advanced/metastatic PD-L1-positive (PD-L1+) TNBC based on the results of the phase III IMpassion130 trial. Furthermore, in 2020, the FDA granted accelerated approval to the PD-1-directed antibody, pembrolizumab, in combination with chemotherapy for advanced/metastatic PD-L1+ TNBC based on the results of the phase III KEYNOTE-355 trial. In 2021, the accelerated approval for pembrolizumab was converted to full approval and the accelerated approval for atezolizumab was voluntarily withdrawn.'

\section{Immunotherapy with PD-(L)1 inhibitors for the treatment of advanced/metastatic breast cancer}

The following sentences have been modified to include information on the withdrawal of atezolizumab: 'At the time of publication, two ICIs were FDA-approved specifically for the treatment of advanced/metastatic TNBC: atezolizumab and pembrolizumab. Both breast cancer-specific approvals are ICIs given in combination with cytotoxic chemotherapy, although the indicated backbone varies between agents and is an ongoing area of investigation.'

The updated text now reads: 'At the time of publication, two ICIs were FDAapproved specifically for the treatment of advanced/metastatic TNBC: atezolizum$\mathrm{ab}$ and pembrolizumab. The indication for atezolizumab was withdrawn in 2021. Both breast cancer-specific approvals were for ICIs given in combination with cytotoxic 
chemotherapy, although the indicated backbone varied between agents and is an ongoing area of investigation.'

A footnote has been added to Table 2 - Trials of ICIs for recurrent/metastatic breast cancer and tissue-agnostic indications, stating, 'The accelerated approval for atezolizumab in combination with nab-paclitaxel was voluntarily withdrawn in 2021.'

The following sentences have been modified to include information on the withdrawal of atezolizumab: 'Accelerated approval was granted in March 2019 for atezolizumab in combination with nab-paclitaxel for treatment of adult patients with PD-L1+ locally advanced or metastatic TNBC, as measured by the VENTANA PD-L1 (SP142) immunohistochemical (IHC) assay and assessed on immune cells (ICs) ${ }^{17}$; additional specifics of PD-L1 testing are described in detail in the Diagnostics and biomarker testing in patients with advanced/ metastatic breast cancer section. Although the approval does not specify line of therapy, data for the clinical activity of atezolizumab beyond the first-line setting is limited.'

The updated text now reads: 'Accelerated approval was granted in March 2019 for atezolizumab in combination with nab-paclitaxel for treatment of adult patients with PD-L1+ locally advanced or metastatic TNBC, as measured by the VENTANA PD-L1 (SP142) immunohistochemical (IHC) assay and assessed on immune cells (ICs) ${ }^{17}$; additional specifics of PD-L1 testing are described in detail in the Diagnostics and biomarker testing in patients with advanced/metastatic breast cancer section. The indication for atezolizumab for TNBC was voluntarily withdrawn in 2021. Although the approval did not specify line of therapy, data for the clinical activity of atezolizumab beyond the first-line setting is limited.'

The following expert panel recommendation has been modified to include information on the withdrawal of atezolizumab: 'At the time of this publication, two companion diagnostics were approved by the FDA for PD-L1 testing in metastatic TNBC: the SP142 assay with IC scoring and the 22C3 assay with tumor and IC scoring by combined positive score. Benefit is seen for adding atezolizumab to nab-paclitaxel in patients with tumors expressing PD-L1 on IC occupying $\geq 1 \%$ of the tumor area by the SP142 assay, and for adding pembrolizumab to chemotherapy in patients with tumors expressing PD-L1 by CPS score $\geq 10$ (LE:2).'

The updated text now reads: ' With the withdrawal of the indication for atezolizumab with nab-paclitaxel in metastatic TNBC, one companion diagnostic is approved by the FDA for PD-L1 testing in metastatic TNBC: the 22C3 assay with tumor and IC scoring by combined positive score. Benefit is seen for adding pembrolizumab to chemotherapy in patients with tumors expressing PD-L1 by CPS score $\geq 10$ (LE:2).'

The following expert panel recommendation has been removed: 'For patients with locally advanced/metastatic TNBC (disease-free interval $\geq 12$ months) and PD-L1 IC+ tumors by IC score $\geq 1$ using the SP142 assay, atezolizumab plus nab-paclitaxel is recommended as one immunotherapy option for first-line treatment (LE:2), based on clinically meaningful OS improvement in IMpassion130.'

The following expert panel recommendation has been removed: 'For patients with locally advanced/metastatic TNBC, it is recommended that atezolizumab should only be added to nab-paclitaxel if tumor-infiltrating ICs expressing PD-L1 occupy $\geq 1 \%$ of the tumor area by the SP142 assay (until PD-L1 assays are harmonized) (LE:2).'

The following expert panel recommendation has been modified to include information on the withdrawal of atezolizumab: 'For patients with locally advanced/metastatic TNBC and PD-L1+ tumors being treated with atezolizumab, nab-paclitaxel is the only chemotherapy backbone that should be used (LE:2).'

The updated recommendation now reads, 'For patients with locally advanced/metastatic TNBC and PD-L1+ tumors being treated with atezolizumab, nab-paclitaxel is the only chemotherapy backbone that has demonstrated activity in randomized clinical trials (LE:2). The indication for atezolizumab in this setting was voluntarily withdrawn in 2021.' 
The following expert panel recommendation has been added to provide guidance on continuation of therapy for patients deriving clinical benefit from atezolizumab based treatment.

The new recommendation reads, 'Patients deriving clinical benefit from atezolizumabbased treatment in the absence of clinically significant toxicity or disease progression should continue on atezolizumab plus nab-paclitaxel rather than change therapy.'

\section{Emerging data on immunotherapy with PD-(L)1 inhibitors for early-stage/locally advanced breast cancer}

The following sentence has been corrected to reflect that full regulatory approval was granted to pembrolizumab in the neoadjuvant setting: 'In July 2021, the FDA granted accelerated approval to pembrolizumab for the treatment of patients with highrisk TNBC in combination with chemotherapy as neoadjuvant treatment and then continued as a single agent as adjuvant treatment after surgery.'

The updated text now reads: 'In July 2021, the FDA granted regular approval to pembrolizumab for the treatment of patients with high-risk TNBC in combination with chemotherapy as neoadjuvant treatment and then continued as a single agent as adjuvant treatment after surgery.'

\section{Diagnostics and biomarker testing in patients with advanced/metastatic breast cancer}

The following sentence has been modified to include information on the withdrawal of atezolizumab: 'Three PD-L1 assays have been designated as 'companion diagnostics' by the FDA, two of which are indicated for breast cancer: the VENTANA PD-L1 (SP142) assay and the PD-L1 IHC 22C3 pharmDx assay.'

The updated text now reads: 'Three PD-L1 assays have been designated as 'companion diagnostics' by the FDA, one of which is indicated for breast cancer: the PD-L1 IHC 22C3 pharmDx assay. The companion diagnostic indication for TNBC for the VENTANA PD-L1 (SP142) assay was withdrawn in 2021.'

The following sentence has been modified to include information on the withdrawal of atezolizumab: 'TNBC is considered 'PD-L1 positive' and the patient eligible to receive atezolizumab per the FDA-approved indication if the tumor shows PD-L1+ ICs occupying $\geq 1 \%$ of the tumor area.'

The updated text now reads, 'TNBC is considered 'PD-L1 positive' and the patient eligible to receive atezolizumab per the formerly FDA-approved indication if the tumor shows PD-L1+ ICs occupying $\geq 1 \%$ of the tumor area. The indication for atezolizumab for TNBC was withdrawn in 2021.'

The following expert panel recommendation has been removed, 'For patients with TNBC being considered for treatment with atezolizumab in combination with nabpaclitaxel, tumor tissue should be tested for PD-L1 by the VENTANA SP142 assay and scored by the IC scoring system, until PD-L1 assays are harmonized (LE:2). A TNBC is PD-L1+ by SP142, and the patient eligible for atezolizumab, with an IC score $\geq 1 \%$.'

Open access This is an open access article distributed in accordance with the Creative Commons Attribution Non Commercial (CC BY-NC 4.0) license, which permits others to distribute, remix, adapt, build upon this work non-commercially, and license their derivative works on different terms, provided the original work is properly cited, appropriate credit is given, any changes made indicated, and the use is non-commercial. See http://creativecommons.org/licenses/by-nc/4.0/.

(C) Author(s) (or their employer(s)) 2021. Re-use permitted under CC BY-NC. No commercial re-use. See rights and permissions. Published by BMJ.

J Immunother Cancer 2021;9:e002597corr1. doi:10.1136/jitc-2021-002597corr1

Check for updates 